\title{
Derived models associated to mice
}

\author{
John R. Steel
}

July 15, 2007

\begin{abstract}
We shall present some results on the the properties of derived models of mice, and on the existence of mice with large derived models. The basic plan of the paper is:

Sections 1-5. Introduction. Preliminary definitions and background material. The iteration independence of the derived model associated to a mouse. The mouse set conjecture in derived models associated to mice.

Sections 6-8 The Solovay sequence in derived models associated to mice. A method of "translating away" extenders overlapping Woodin cardinals in mice.

Sections 9-14. Some partial results on capturing $\Sigma_{1}^{2}$ truths in $\mathrm{AD}^{+}$models by mice. (That is, partial results on the mouse set conjecture.)

Section 15 A theorem of Woodin on the consistency strength of $\mathrm{AD}^{+}+\theta_{0}<\theta$.

Section 16 The global mouse set conjecture implies its local version.

Section 17 Capturing sets of reals in $\mathbb{R}$-mice.
\end{abstract}

\section{Introduction}

In this paper, we shall present some results on the the properties of derived models of mice, and on the existence of mice with large derived models. ${ }^{1}$

In order to motivate what follows, let us recall some well-known Holy Grails of inner model theory:

Conjecture 1.0.1 Each of the following statements implies the existence of an $\omega_{1}+1$ iterable mouse with a superstrong cardinal:

\footnotetext{
${ }^{1}$ The paper began as a set of notes which accompanied some lectures at the workshop Computational Prospects of Infinity, held at the Institute for Mathematical Sciences of the National University of Singapore, in June 2005. The author wishes to thank the conference organizers and the Institute for their gracious hospitality.
} 
(1) There is a supercompact cardinal,

(2) There is a strongly compact cardinal,

(3) PFA,

(4) $\mathrm{CH}$ holds, and there is a homogeneous pre-saturated ideal on $\omega_{1}$,

(5) $\mathrm{AD}_{\mathbb{R}}$ holds, and $\theta$ is regular.

This list could be lengthened substantially. In all cases, we can prove the statement in question implies that there is an iterable mouse with infinitely many Woodin cardinals. In cases (2)-(5), we cannot yet produce a mouse with a Woodin limit of Woodin cardinals. (Case (1) is special; here the best partial results are those of [13], which reach a bit past a Woodin limit of Woodins.)

The partial results we have in cases (2)-(5) come from the core model induction method. In this method (due to Woodin), one uses core model theory to construct new mice, while using descriptive set theory to keep track of the degree of correctness and the complexity of the iteration strategies of the mice one has constructed. See [4], [32], and [27] for examples of core model inductions. We suspect that even in case (1), one will not be able to go significantly beyond where we are now without bringing in core model induction ideas.

Core model inductions reaching infinitely many Woodins, or further, rely on a key step in which one passes from some kind of hybrid mouse to an ordinary $L[\vec{E}]$ mouse. The hybrid mouse $\mathcal{H}$ will have a small number of Woodin cardinals, but these cardinals will be Woodin with respect to predicates coding up a lot of information. The $L[\vec{E}]$ mouse $\mathcal{M}$ is built by a full background extender construction inside $\mathcal{H}$, and may have all sorts of Woodin cardinals, even cardinals strong past a Woodin, and presumably even superstrong cardinals. We sometimes call the ordinary $L[\vec{E}]$ mice $m s$-mice; they are mice in the sense of [19], except that we allow them to be relativised by putting some arbitrary transitive set $y$ at the bottom, in which case we speak of an ms-mouse over $y$. (See e.g. [27].) The following conjecture is the basic test problem for our ability to translate hybrid mice into ordinary ms-mice.

Conjecture 1.0.2 ((Mouse Set Conjectures (MSC))) Assume $\mathrm{AD}^{+}$, and that there is no $\omega_{1}$-iteration strategy for a mouse with a superstrong cardinal; then for any countable transitive set $y$,

(I) if $x \subseteq y$, and $x$ is ordinal definable from parameters in $y \cup\{y\}$, then there is an $\omega_{1}$-iterable ms-mouse $\mathcal{M}$ over y such that $x \in \mathcal{M}$, and

(II) if $\exists A \subseteq \mathbb{R}(H C, \in, A) \models \varphi[y]$, then there is an $\omega_{1}$-iterable ms-mouse $\mathcal{M}$ over $y$, and a $\lambda$ such that

$$
\mathcal{M}=\lambda \text { is a limit of Woodin cardinals, }
$$


and

$$
\mathcal{M} \models \exists A \in \operatorname{Hom}_{<\lambda}((H C, \in, A) \models \varphi[y]) .
$$

MSC2 is equivalent to asserting that the $\Sigma_{1}^{2}$ fact $\exists A \subseteq \mathbb{R}(\mathrm{HC}, \in, A) \models \varphi[y]$ holds in the derived model of $\mathcal{M}$ below $\lambda$. It is easy to show that MSC2 implies MSC1, and not too hard to show that MSC1 implies MSC2. We shall therefore use MSC to stand for either MSC1 or MSC2, in situations where we don't need to make a distinction.

Clearly, the conclusion of MSC1 is an equivalence: if $x$ is in an iterable mouse over $y$, then $x \in \mathrm{OD}(y \cup\{y\})$. In MSC2, one seems to need to add something about the iteration strategy for $\mathcal{M}$ in order to conclude that any $\Sigma_{1}^{2}$ statement true in the derived model of $\mathcal{M}$ is actually true. This can be done in a natural way. (It is enough that the strategy be "good" in the sense described at the beginning of section 3.)

MSC is known for a certain initial segment of the Wadge hierarchy. Woodin has shown:

Theorem 1.1 (Woodin, late 90's) MSC holds if the hypothesis is strengthened to $\mathrm{AD}^{+}$ plus there is no iteration strategy for an ms-mouse $\mathcal{M}$ such that for some $\lambda$,

$$
\mathcal{M} \models \lambda \text { is a limit of Woodin cardinals , }
$$

and

$$
\{\kappa \mid \kappa \text { is }<\lambda \text {-strong in } \mathcal{M}\} \text { has order type } \lambda \text {. }
$$

The proof builds on Woodin's proof of MSC under the stronger hypothesis that there is no iteration strategy for a nontame mouse. That proof is written up in [23]. There are many additional difficulties in dealing with ms-mice having extenders overlapping Woodin cardinals, however. An $\omega_{1}$ iteration strategy for a countable premouse is essentially a set of reals, so the hypothesis of Theorem 1.1 asserting that there is no $\omega_{1}$ iteration strategy for a mouse of the type amounts to a restriction to the initial segment of the Wadge hierarchy below the Wadge-least such strategy. A roughly equivalent way to put this restriction is: there is no proper initial segment $\Gamma$ of the Wadge hierarchy such that $L(\Gamma, \mathbb{R}) \models A D_{\mathbb{R}}+D C$.

More recently, Itay Neeman and the author have shown

Theorem 1.2 (Neeman-Steel, 2004) MSC holds if the hypothesis is strengthened to $\mathrm{AD}^{+}$ plus there is no iteration strategy for an ms-mouse $\mathcal{M}$ such that for some $\lambda$,

$$
\mathcal{M} \models \lambda \text { is a limit of Woodin cardinals, }
$$

and for some $\kappa<\lambda$,

$$
\mathcal{M} \models \kappa \text { is }<\lambda \text { strong, and a limit of }<\lambda \text {-strongs. }
$$


Although the large cardinal reached here is only slightly beyond the large cardinals reached in 1.1, the proof has some new ideas, and seems somewhat simpler, and closer to Woodin's original argument in the tame mouse case. In sections 9 through 14 , we shall give these arguments in the special case that $V$ is the minimal model of $\mathrm{AD}^{+}+\theta_{0}<\theta$. Pretty much all the ideas occur in this case. In section 15, we use this work to re-prove a theorem of Woodin which identifies the consistency strength of $\mathrm{AD}^{+}+\theta_{0}<\theta$.

The mouse set conjectures ask us to construct ms-mice with given derived models. This leads naturally to the question: what can one say about the derived model of a mouse? (See [25] for an exposition of the basic facts about the derived model construction.) This will be the focus of sections 3 through 8 . We shall show that for certain "tractable" $\mathcal{M}, \lambda$ such that $\mathcal{M}$ is a mouse and $\lambda$ is a limit of Woodins in $\mathcal{M}$, the derived model $\mathcal{D}(\mathcal{M}, \lambda)$ of $\mathcal{M}$ at $\lambda$ satisfies MSC. We shall show that for these tractable pairs $(\mathcal{M}, \lambda)$, there is a canonical derived model of an iterate of $\mathcal{M}$ whose reals are precisely the reals in $V$. Finally, we shall investigate the Solovay sequence $\left\langle\theta_{\alpha}\right\rangle$ of various $\mathcal{D}(\mathcal{M}, \lambda)$. For example, letting $M_{\text {wlim }}^{\sharp}$ be the minimal active mouse with a Woodin limit of Woodin cardinals, we shall show

Theorem 1.3 (Closson, Steel) Let $\lambda$ be the Woodin limit of Woodins in $M_{\text {wlim }}^{\sharp}$; then

$$
\mathcal{D}\left(M_{w l i m}^{\sharp}, \lambda\right) \models \theta=\theta_{\theta} .
$$

We do not know what the cofinality of $\theta$ is in the model of 1.3 . Possibly $\theta$ is regular here, in which case $A D_{\mathbb{R}}$ plus " $\theta$ is regular" is much weaker than conjecture 1.0.1(5) would have it.

In section 16, we combine ideas from the two parts of the paper in order to show that MSC implies a reasonably fine local strengthening of itself. In section 17, we show that MSC implies that every $\mathrm{OD}(\mathbb{R})$ set of reals is in a countably iterable mouse over $\mathbb{R}$.

This paper is far from self-contained. We have gathered together a fair amount of background material for the reader's convenience, but in order to keep this document to a reasonable size, we have also simply pointed to the literature at many points. Unfortunately, some of the key results have not been written up in full anywhere. We have sketched proofs of such results, or given references for parts of them, when it was feasible to do so.

\section{Some background and preliminaries}

\subsection{Homogeneously Suslin sets}

The good sets of reals, from the point of view of descriptive set theory, are the homogeneously Suslin sets.

Definition 2.1 A homogeneity system with support $Z$ is a function $\bar{\mu}$ such that for all $s, t \in \omega^{<\omega}$, 
1. $\mu_{t}$ is a countably complete ultrafilter concentrating on $Z^{\mathrm{dom}(t)}$, and

2. $s \subseteq t \Rightarrow \mu_{t}$ projects to $\mu_{s}$.

If all $\mu_{t}$ are $\kappa$-complete, then we say $\bar{\mu}$ is $\kappa$-complete.

Definition 2.2 If $\bar{\mu}$ is a homogeneity system, then for $x \in \omega^{<\omega}$,

$$
\begin{aligned}
x \in S_{\bar{\mu}} \Leftrightarrow \quad \text { the tower of measures }\left\langle\mu_{x \uparrow n} \mid n<\omega\right\rangle \\
\quad \text { is countably complete. }
\end{aligned}
$$

Let

$$
\begin{gathered}
\operatorname{Hom}_{\kappa}=\left\{S_{\bar{\mu}} \mid \bar{\mu} \text { is } \kappa \text {-complete }\right\}, \\
\operatorname{Hom}_{<\lambda}=\bigcap_{\kappa<\lambda} \operatorname{Hom}_{\kappa}, \\
\operatorname{Hom}_{\infty}=\bigcap_{\kappa \in \mathrm{OR}} \operatorname{Hom}_{\kappa} .
\end{gathered}
$$

A set of reals is homogeneously Suslin just in case it is in $\mathrm{Hom}_{\kappa}$, for some $\kappa$. Although not literally stated in the paper, one of the main results of Martin [7] is that every homogeneously Suslin set is determined. There are no interesting homogeneously Suslin sets unless there are measurable cardinals. For the most part, we shall be working under the assumption that there are infinitely many Woodin cardinals, in which case one has:

Theorem 2.3 Let $\lambda$ be a limit of Woodin cardinals; then

(a) $\mathrm{Hom}_{<\lambda}$ is closed under complements and real quantification ([10]),

(b) every $\mathrm{Hom}_{<\lambda}$ set has a $\operatorname{Hom}_{<\lambda}$ scale ([25]).

Feng, Magidor, and Woodin ([1]) introduced an important notion which turns out to be equivalent to homogeneity in the presence of Woodin cardinals.

Definition 2.4 Let $T$ and $T^{*}$ be trees on $\omega \times X$ and $\omega \times Y$ respectively; then $T$ and $T^{*}$ are $\kappa$-absolute complements iff whenever $G$ is $V$-generic over a poset of size $\leq \kappa$, then $V[G] \models p[T]=\mathbb{R} \backslash p\left[T^{*}\right]$. We say that $A \subseteq \mathbb{R}$ is $\kappa$-universally Baire (or $\kappa$-UB) iff $A=p[T]$ for some tree $T$ for which there is a $\kappa$-absolute complement. 
The results of [9], [30], and [10] show that if $\lambda$ is a limit of Woodin cardinals, then the Hom $_{<\lambda}$ sets are precisely the $<\lambda$-universally Baire sets. (See [25].) The theorem and this equivalence also hold for $\lambda=\infty=$ OR.

Stronger large cardinal hypotheses imply stronger closure properties of $\mathrm{Hom}_{<\lambda}$. For example, Woodin has shown that if $\lambda$ is a limit of Woodin cardinals, and there is a measurable cardinal above $\lambda$, then for any $A \in \operatorname{Hom}_{<\lambda}, A^{\sharp} \in \mathrm{Hom}_{<\lambda}$. However, no large cardinal hypothesis is known to imply that sets of reals definable using quantification over $\mathrm{Hom}_{<\lambda}$ must be $\mathrm{Hom}_{<\lambda}$, or even good in some weaker sense. The inner model theory for such a large cardinal hypothesis would be significantly different from the one we have now, as we explain in the next subsection.

\section{2 $\operatorname{Hom}_{\infty}$ iteration strategies}

Let $\mathcal{M}$ be a ms-premouse: a model of the form $\left(J_{\alpha}[\vec{E}], \in, \vec{E}, F\right)$, where $\vec{E} \frown F$ is a fine extender sequence. ([19].) In the iteration game $G(\mathcal{M}, \theta)$, I and II cooperate to build an iteration tree $\mathcal{T}$ on $\mathcal{M}$ :

- I extends $\mathcal{T}$ at successor steps by applying an extender from the current model to some, possibly earlier, model. The resulting ultrapower is the new current model.

- At limit steps II picks a cofinal branch $b$, and $\lim _{\alpha \in b} \mathcal{M}_{\alpha}$ becomes the current model.

II wins if, after $\theta$ moves, no illlfounded model has been produced. See [19] for a more complete description of $G(\mathcal{M}, \theta)$.

Definition 2.5 $A$-iteration strategy for $\mathcal{M}$ is a winning strategy for II in $G(\mathcal{M}, \theta) . \mathcal{M}$ is $\theta$-iterable if there is such an iteration strategy.

Notice that if $\mathcal{M}$ is countable, then an $\omega_{1}$-iteration strategy for $\mathcal{M}$ is essentially a set of reals. "Good" $\mathcal{M}$ (the "standard" ones), have $\omega_{1}$-iteration strategies which are Hom ${ }_{\infty}$. This implies $\theta$-iterability, for all $\theta$.

For $L[\vec{E}]$-models in the region where we have a theory ( can prove iterability),

$$
\begin{gathered}
x<^{L[\vec{E}]} y \Leftrightarrow \quad \exists \mathcal{M}\left(\mathcal{M} \text { has a } \text { Hom }_{\infty}\right. \text { iteration strategy } \\
\text { and } \left.\mathcal{M} \models x<^{L[\vec{E}]} y\right) .
\end{gathered}
$$

This can be used to show that the large cardinal hypotheses true in these models are compatible with there being a wellorder of $\mathbb{R}$ in $L\left(\mathbb{R}, \mathrm{Hom}_{\infty}\right)$. 


\subsection{The derived model}

Given $A \in \operatorname{Hom}_{\kappa}$, as witnessed by both $\bar{\mu}$ and $\bar{\nu}$, and $G$ which is $V$-generic for a poset of size $<\kappa$, we have

$$
\left(S_{\bar{\mu}}\right)^{V[G]}=\left(S_{\bar{\nu}}\right)^{V[G]} .
$$

So we write $A^{V[G]}$ for the common value of all $\left(S_{\bar{\mu}}\right)^{V[G]}$ such that $A=S_{\bar{\mu}}$.

Now let $\lambda$ be a limit of Woodin cardinals, and $G$ be $V$-generic for $\operatorname{Col}(\omega,<\lambda)$, and set

$$
\begin{aligned}
\mathbb{R}_{G}^{*} & =\bigcup_{\alpha<\lambda} \mathbb{R} \cap V[G \uparrow \alpha], \\
\operatorname{Hom}_{G}^{*} & =\left\{A^{*} \mid \exists \alpha<\lambda\left(A \in \operatorname{Hom}_{<\lambda}^{V[G\lceil\alpha]}\right)\right\}
\end{aligned}
$$

where $A^{*}=\bigcup_{\beta<\lambda} A^{V[G \uparrow \beta]}$. Note that one has

$$
\operatorname{Hom}_{G}^{*}=\left\{p[T] \cap \mathbb{R}_{G}^{*} \mid \exists \alpha<\lambda(V[G \uparrow \alpha] \models T \text { has an }<\lambda \text {-absolute complement })\right\} \text {. }
$$

\section{Theorem 2.6 (Derived model theorem, Woodin 1987)}

(1) $L\left(\mathbb{R}^{*}, \mathrm{Hom}^{*}\right) \models \mathrm{AD}^{+}$.

(2) Hom $^{*}=\left\{A \subseteq \mathbb{R}^{*} \mid A, \mathbb{R} \backslash A\right.$ have scales in $L\left(\mathbb{R}^{*}\right.$, Hom $\left.\left.^{*}\right)\right\}$.

If $\mathcal{M}=\lambda$ is a limit of Woodins, and a reasonable fragment of ZFC, then we write

$$
D(\mathcal{M}, \lambda)
$$

for the associated derived model. This is analogous to the $V^{P}$ notation, since we have not specified a generic. Since the forcing is homogeneous, the theory of $D(\mathcal{M}, \lambda)$ does not depend on a generic. We shall speak of "realizations" of $D(\mathcal{M}, \lambda)$ if we have specified a generic $G$. Note that only $\mathbb{R}_{G}^{*}$, not all of $G$, is needed to realize $D(\mathcal{M}, \lambda)$.

Remark 2.7 This is the "old" derived model; the model is always of the form $L(\Gamma, \mathbb{R})$, where $\Gamma$ is the class of its Suslin-co-Suslin sets. Not all models of $\mathrm{AD}^{+}$have this form. Woodin has shown that the Suslin-co-Suslin sets of any $\mathrm{AD}^{+}$model can be realized as the Suslin-co-Suslin sets of a derived model, however, and given a variant construction which hits all models of $\mathrm{AD}^{+}$plus $V=L(P(\mathbb{R}))$ in full.

If $\mathcal{M}=M_{\omega}$, the minimal mouse with $\omega$ Woodins, then letting $\lambda$ be their $\sup , D(\mathcal{M}, \lambda)=$ $L\left(\mathbb{R}^{*}\right)$. On the other hand, for stronger $\mathcal{M}, D(\mathcal{M}, \lambda)$ may be larger. 
Definition $2.8\left(\mathrm{AD}^{+}\right.$.) For $A \subseteq \mathbb{R}, \theta(A)$ is the least ordinal $\alpha$ such that there is no surjection of $\mathbb{R}$ onto $\alpha$ which is ordinal definable from $A$ and a real. We set

$$
\begin{aligned}
\theta_{0} & =\theta(\emptyset), \\
\theta_{\alpha+1} & =\theta(A), \text { for any (all) A of Wadge rank } \theta_{\alpha}, \\
\theta_{\lambda} & =\bigcup_{\alpha<\lambda} \theta_{\alpha} .
\end{aligned}
$$

$\theta_{\alpha+1}$ is defined iff $\theta_{\alpha}<\Theta$. Note $\theta(A)<\Theta$ iff there is some $B \subseteq \mathbb{R}$ such that $B \notin$ $\mathrm{OD}(\mathbb{R} \cup\{A\})$. In this case, $\theta(A)$ is the least Wadge rank of such a $B$.

Theorem 2.9 (Woodin, mid 80's) Assume $\mathrm{AD}^{+}$, and suppose $A$ and $\mathbb{R} \backslash A$ admit scales; then

(a) All $\Sigma_{1}^{2}(A)$ sets of reals admit scales, and

(b) All $\Pi_{1}^{2}(A)$ sets admit scales iff $\theta(A)<\Theta$.

Theorem 2.10 (Martin, Woodin, mid 80's) Assume $\mathrm{AD}^{+}$; then the following are equivalent:

(1) $\mathrm{AD}_{\mathbb{R}}$

(2) Every set of reals admits a scale,

(3) $\Theta=\theta_{\lambda}$, for some limit $\lambda$.

Sadly, the proofs of these theorems have never fully appeared. There is a good deal on consequences of $\mathrm{AD}^{+}$in [29]. There is most of a proof that $\operatorname{Scale}\left(\Sigma_{1}^{2}\right)$ holds in derived models in $[25]$, section 7 .

Theorem 2.11 (Woodin, 1988, 2000) Suppose $\lambda$ is a limit of Woodins, and $L\left(\mathbb{R}^{*}\right.$, Hom $\left.^{*}\right)$ is a derived model at $\lambda$; then

(a) $\exists \kappa<\lambda(\kappa$ is $<\lambda$-strong $) \Rightarrow L\left(\mathbb{R}^{*}\right.$, Hom $\left.^{*}\right) \models \theta_{0}<\Theta$.

(b) $\lambda$ is a limit of $\kappa$ which are $<\lambda$-strong $\Rightarrow L\left(\mathbb{R}^{*}\right.$, Hom $\left.^{*}\right) \mid=\Theta=\theta_{\alpha}$, for some limit $\alpha$.

(c) $\lambda$ is an inaccessible limit of $\kappa$ which are $<\lambda$-strong $\Rightarrow L\left(\mathbb{R}^{*}, \mathrm{Hom}^{*}\right)=\Theta=\theta_{\alpha}$, where $\operatorname{cof}(\alpha) \geq \omega_{1}$

(d) $\exists \kappa<\lambda(\kappa$ is $\lambda$-supercompact $) \Rightarrow L\left(\mathbb{R}^{*}, H^{*}{ }^{*}\right) \models \mathrm{AD}_{\mathbb{R}}+\Theta$ is regular.

Remarks. 
(i) Parts (a)-(c) are proved in [25]. Part (d) has not been written up.

(ii) The hypotheses of (a)-(c) follow from $\lambda$ being a Woodin limit of Woodin cardinals.

(iii) The hypothesis of (b), that there is a $\lambda$ which is a limit of Woodins and of $<\lambda$-strongs, is called the $A D_{\mathbb{R}}$ hypothesis. Woodin has shown the $A D_{\mathbb{R}}$ hypothesis is equiconsistent with $A D_{\mathbb{R}}$. One direction is (b) above.

(iv) There is a big jump going from (c) to (d). How strong is $A D_{\mathbb{R}}+\Theta$ regular ?

(v) There are other ways of calibrating the strength of determinacy models. One can look at the complexity of the games of length $\omega_{1}$ which are determined in the model. One can look at the complexity of the mice which have iteration strategies in the model.

\subsection{Iterations to make $\mathbb{R}^{V}=\mathbb{R}^{*}$}

Let $\mathcal{M}$ be countable and $\left(\omega_{1}+1\right)$-iterable, with $\lambda$ a limit of Woodin cardinals of $\mathcal{M}$. Working in $\left.V^{\operatorname{Col}(\omega, \mathbb{R}}\right)$, we can form an $\mathbb{R}$-genericity iteration (of $\mathcal{M}$, below $\lambda$ ), that is, a sequence

$$
I=\left\langle\mathcal{T}_{n} \mid n<\omega\right\rangle
$$

such that the $\mathcal{T}_{n}$ are iteration trees whose composition

$$
\mathcal{T}=\oplus_{n} \mathcal{T}_{n}
$$

is a normal, nondropping iteration tree on $\mathcal{M}$, with

$$
\mathcal{M}_{\infty}^{I}=\lim _{n} \mathcal{M}_{n}^{I}
$$

the direct limit along the main branch of $\mathcal{T}$ (where $\mathcal{M}_{n}^{I}$ is the base model of $\mathcal{T}_{n}$, and the last model of $\mathcal{T}_{n-1}$ if $n>0$ ), being such that $\mathbb{R}^{V}$ is the reals of a symmetric collapse over $\mathcal{M}_{\infty}^{I}$ below $\lambda_{\infty}^{I}$, the image of $\lambda$. We write

$$
\operatorname{Hom}_{I}^{*}=\bigcup\left\{p[T] \cap \mathbb{R}^{V} \mid \exists x \in \mathbb{R}^{V}\left(\mathcal{M}_{\infty}^{I} \models T \text { is }<\lambda \text { absolutely complemented }\right)\right\},
$$

and

$$
D\left(\mathcal{M}_{\infty}^{I}, \lambda_{\infty}^{I}\right)=L\left(\mathbb{R}^{V}, \operatorname{Hom}_{I}^{*}\right)
$$

for the derived model of $\mathcal{M}_{\infty}^{I}$ at $\lambda_{\infty}^{I}$ whose set of reals is $\mathbb{R}^{*}=\mathbb{R}^{V}$. 


\subsection{Premice over a set}

We shall be most interested in ms premice over countable, transitive sets $a$ which are selfwellordered, where $a$ is self-wellordered iff there is a wellorder of $a$ which is rudimentary in $a$. If $\mathcal{P}$ is a premouse over such an $a$, it satisfies $A C$, and the usual Los theorem for finestructural ultrapowers, etc. We will sometimes speak of a mouse over $a$ when $a \in \mathrm{HC}$ is not transitive. In this case, we are really speaking of a mouse over the transitive closure of $a \cup\{a\}$.

Nevertheless, the notion of an $a$-premouse makes sense for any transitive set $a$, selfwellordered or not, and we shall phrase some definitions in this generality. The reader should see [24] for a discussion of the elementary properties of such premice, in the representative special case that $a=\mathrm{HC}$. The main thing is that if $\mathcal{M}$ is an $a$-premouse with top extender $E$, and $f:(a \times \xi) \rightarrow E_{b}$ with $\xi<\operatorname{crit}(E)$ and $f \in \mathcal{M}$, then $\bigcap \operatorname{ran}(f) \in E_{b}$. This implies that $i_{E}$ is the identity on $a \cup\{a\}$, and that we have Los' theorem for $\Sigma_{n}$ ultrapowers, whenever $\operatorname{crit}(E)<\rho_{n}^{\mathcal{M}}=$ least $\rho$ such that there is a new $\Sigma_{n}^{\mathcal{M}}$ subset of $a \times \rho$. These properties imply that if $g$ is $\operatorname{Col}(\omega, a)$-generic over $\mathcal{M}$, then $\mathcal{M}[g]$ can be regarded as an ordinary premouse over the swo $\langle a, g\rangle$. This last fact summarizes what it is to be an $a$-premouse: you become an ordinary premouse when a wellorder of $a$ is added generically.

\section{$3 \quad$ Iteration independence for derived models of mice}

In this section we consider derived models of ms-mice.

An $\mathbb{R}$-genericity iteration $I$ of a countable $\mathcal{M}$ cannot belong to $V$, unless $\lambda$ happens to be measurable in $\mathcal{M}$. Nevertheless, in many interesting cases, the derived model $D\left(M_{\infty}^{I}, \lambda_{\infty}^{I}\right)$ is in $V$.

We need a minor technical strengthening of iterability in order to state this result precisely. Let us call an $\omega_{1}+1$ iteration strategy $\Sigma$ for $\mathcal{M}$ good iff whenever $\mathcal{T}$ is a countable normal iteration tree played by $\Sigma$ with last model $\mathcal{P}$, and $\beta<\operatorname{lh}(\mathcal{T})$, then the phalanx obtained from $\Phi(\mathcal{T} \uparrow(\beta+1))$ obtained by replacing its last model $\mathcal{M}_{\beta}^{\mathcal{T}}$ with $\mathcal{P}$ is such that $\Psi$ is $\omega_{1}+1$-iterable. All our iterability proofs give good strategies, but we do not see how to show that every $\omega_{1}+1$, or even $\left(\omega, \omega_{1}+1\right)$, iteration strategy is good.

Proposition 3.0.1 Let $\mathcal{M}$ be $\omega$-sound and project to $\omega$, and let $\Sigma$ be a good $\omega_{1}+1$ iteration strategy for $\mathcal{M}$. Let $I$ be an $\mathbb{R}$-genericity iteration of $\mathcal{M}$ below $\lambda$ such that $I$ is played according to $\Sigma$; then every set in $\mathrm{Hom}_{I}^{*}$ is projective in $\Sigma \uparrow H C$. In particular, $\mathrm{Hom}_{I}^{*} \subseteq V$.

Proof. Let $A \in \operatorname{Hom}_{I}^{*}$, as witnessed by $T \in \mathcal{M}_{\infty}^{I}[x]$, where $x \in \mathbb{R}^{V}$. Let $S \in \mathcal{M}_{\infty}^{I}[x]$ absolutely complement $T$ for forcings of size $<\lambda_{\infty}^{I}$. Let $I=\left\langle\mathcal{T}_{n} \mid n<\omega\right\rangle$, and let $n<\omega$ be large enough that, letting

$$
\mathcal{N}=\text { last model of } \mathcal{T}_{n}
$$


and

$$
\pi: \mathcal{N} \rightarrow \mathcal{M}_{\infty}^{I}
$$

be the canonical embedding, we have that for $\kappa=\operatorname{crit}(\pi)$,

$$
x \in \mathcal{N}[g],
$$

where $g \in V$ is $\mathcal{N}$-generic over $\operatorname{Col}(\omega, \eta)$, for some $\eta<\kappa$. Note that we can lift $\pi$ to an embedding of $\mathcal{N}[g]$ into $\mathcal{M}_{\infty}^{I}[g]$, which we also call $\pi$. We assume that $n$ has been chosen large enough that the $g$-terms for $T$ and $S$ have pre-images in $\mathcal{N}$, and let then

$$
\pi((\bar{T}, \bar{S}))=(T, S)
$$

Working now in $V$, we can define $A$ as follows: for $y \in \mathbb{R}$,

$$
\begin{aligned}
y \in A \Leftrightarrow \quad \exists \mathcal{U} \in \operatorname{HC}\left(\left(\oplus_{k \leq n} \mathcal{T}_{k}\right) \oplus \mathcal{U}\right. \text { is a normal iteration tree } \\
\\
\text { on } \mathcal{M} \text { played by } \Sigma, \text { and } y \in p\left[i^{\mathcal{U}}(\bar{T})\right] .
\end{aligned}
$$

On the right hand side of this equivalence, " $i \mathcal{U}$ " stands for the lift of the canonical embedding of $\mathcal{U}$, so that $i^{\mathcal{U}}: \mathcal{N}[g] \rightarrow \mathcal{P}[g]$, where $\mathcal{P}$ is the last model of $\mathcal{U}$.

The $\Rightarrow$ direction of the equivalence follows by taking $\mathcal{U}=\bigoplus_{n<k \leq m} \mathcal{T}_{k}$, for $m$ sufficiently large. For the other direction, suppose $\mathcal{U}$ is as on the right hand side, and toward contradiction, that $y \notin A$. We can then find an iteration tree $\mathcal{W}$ on $\mathcal{N}$ such that $\oplus_{k \leq n} \mathcal{T}_{k} \oplus \mathcal{W}$ is a normal tree by $\Sigma$ with $y \in p\left[i^{\mathcal{W}}(\bar{S})\right]$, where $i^{\mathcal{W}}$ is the lift of the canonical embedding of $\mathcal{W}$. ( Take $\mathcal{W}=\oplus_{n<k \leq m} \mathcal{T}_{k}$, for $m$ sufficiently large.) Let $\mathcal{P}$ and $\mathcal{Q}$ be the last models of $\mathcal{U}$ and $\mathcal{W}$ respectively, and let $\Psi$ and $\Gamma$ be the phalanxes obtained from $\Phi\left(\left(\oplus_{k \leq n} \mathcal{T}_{k}\right)\right.$ by replacing its last model $\mathcal{N}$ with $\mathcal{P}$ and $\mathcal{Q}$, respectively. Since $\Sigma$ is good, $\Psi$ and $\Gamma$ are $\omega_{1}+1$ iterable, so we can coiterate them.

Standard arguments using the fact that $\mathcal{M}$ is $\omega$-sound and projects to $\omega$ show that the last model on the two sides in the comparison of $\Psi$ and $\Gamma$ is the same, call it $\mathcal{R}$, and is above $\mathcal{P}$ and $\mathcal{Q}$ respectively, and the branches $\mathcal{P}$-to- $\mathcal{R}$ and $\mathcal{Q}$-to- $\mathcal{R}$ do not drop. Thus we have

$$
\mathcal{N} \stackrel{i^{\mathcal{U}}}{\longrightarrow} \mathcal{P} \stackrel{j}{\longrightarrow} \mathcal{R}
$$

and

$$
\mathcal{N} \stackrel{i^{\mathcal{W}}}{\longrightarrow} \mathcal{Q} \stackrel{k}{\longrightarrow} \mathcal{R}
$$

where $j$ and $k$ are given by the comparison of $\Psi$ and $\Gamma$. Let

$$
\sigma: \mathcal{M} \rightarrow \mathcal{N}
$$

be given by $\oplus_{k \leq n} \mathcal{T}_{k}$, and let $\nu$ be the sup of the generators of extenders in this tree used on $\mathcal{M}$-to- $\mathcal{N}$. Every element of $\mathcal{N}$ is definable from points in $\operatorname{ran}(\sigma)$ and ordinals $<\nu$. On the other hand, $j \circ i^{\mathcal{U}}$ and $k \circ i^{\mathcal{W}}$ are the identity on $\nu$, and agree on $\operatorname{ran}(\sigma)$. Thus

$$
j \circ i^{\mathcal{U}}=k \circ i^{\mathcal{W}} \text {. }
$$


But then $y \in p\left[i^{\mathcal{U}}(\bar{T}]\right.$, so $y \in p\left[j\left(i^{\mathcal{U}}(\bar{T})\right)\right]$, so $y \in p\left[k\left(i^{\mathcal{W}}(\bar{T})\right)\right]$. On the other hand, $y \in p\left[i^{\mathcal{W}}(\bar{S}]\right.$, so $y \in p\left[k\left(i^{\mathcal{W}}(\bar{S})\right)\right]$. Thus $k\left(i^{\mathcal{W}}(\bar{T})\right)$ and $k\left(i^{\mathcal{W}}(\bar{S})\right)$ do not have disjoint projections, although it is satisfied by $\mathcal{R}[g]$ that their projections are disjoint. This contradicts the wellfoundedness of $\mathcal{R}[g]$.

Remark 3.1 (i) Under sufficiently strong hypotheses, one can expect that the iteration strategy $\Sigma$ of 3.0.1 will be $\mathrm{Hom}_{\infty}$, in which case we get that $\operatorname{Hom}_{I}^{*}$ is a Wadge initial segment of $\operatorname{Hom}_{\infty}$.

(ii) It is easy to see that the strategy $\Sigma$ is not itself in $\operatorname{Hom}_{I}^{*}$, as otherwise $\mathcal{M}$ would be ordinal definable over $D\left(\mathcal{M}_{\infty}^{I}, \lambda_{\infty}^{I}\right)$, hence in $\mathcal{M}_{\infty}^{I}$, hence in $\mathcal{M}$. Given that $\Sigma$ is $\operatorname{Hom}_{\infty}$, we conjecture that if $\mathcal{M}$ is a sharp mouse which reconstructs itself below $\lambda$ (see 3.3, 3.4 below), and $\lambda$ is a limit of Woodin cardinals in $\mathcal{M}$ such that every extender on the $\mathcal{M}$-sequence except the last one has length $<\lambda$, then $\Sigma$ is in any scaled pointclass closed under $\exists^{\mathbb{R}}$ and $\neg$ which properly includes $\operatorname{Hom}_{I}^{*}$. This amounts to asserting that any new Suslin sets beyond $\operatorname{Hom}_{I}^{*}$ bring with them new $\Sigma_{1}^{2}$ facts, and that a local MSC holds just beyond $\mathrm{Hom}_{I}^{*}$. One needs to assume that $\mathcal{M}$ is a sharp mouse here.

(iii) Let $\mathcal{T}$ be a non-dropping iteration tree on $\mathcal{M}$ played by $\Sigma$, with last model $\mathcal{N}$, and let $\eta<\delta$ be $\mathcal{N}$-cardinals, with $\nu\left(E_{\alpha}\right)^{\mathcal{T}}<\eta$ for all $\alpha$, so that $\mathcal{N}$ is $\eta$-sound. We write $\Sigma \mid(\mathcal{T},[\eta, \delta])$ for the restriction of $\Sigma$ to iteration trees on $\mathcal{N} \mid \delta$ which use only extenders with critical point $>\eta$, and do not drop anywhere. (Such trees are normal continuations of $\mathcal{T}$.) We call $\Sigma \mid(\mathcal{T},[\eta, \delta])$ a window-based fragment of $\Sigma$. We call the window $[\eta, \delta]$ short if there are no Woodin cardinals of $\mathcal{N}$ strictly between $\eta$ and $\delta$, and in this case call $\Sigma \mid(\mathcal{T},[\eta, \delta])$ a small fragment of $\Sigma$. The proof of 3.0.1 then shows that every set in $\operatorname{Hom}_{I}^{*}$ is projective in some small fragment of $\Sigma$. In some cases, we can show that all the small fragments of $\Sigma$ are in $\mathrm{Hom}_{I}^{*}$, so that they are Wadge cofinal, and use this to compute the cofinality of $\theta$ in $D(\mathcal{M}, \lambda)$. See 7.3. We do not know whether this is true in general. In particular, we do not know whether if $\Sigma$ is the unique $\omega_{1}$ strategy for the sharp of the minimal model with a Woodin limit of Woodins, then all small fragments of $\Sigma$ are in the associated derived model.

(iv) In general, the initial segment based fragments of $\Sigma$, i.e., the fragments based on windows of the form (empty tree, $[0, \delta]$ ), are not all in $D\left(\mathcal{M}_{\infty}^{I}, \lambda_{\infty}^{I}\right)$. For suppose the cofinality of $\lambda$ is not measurable in $\mathcal{M}$, so all iteration maps are continuous at $\lambda$. Suppose also $D(\mathcal{M}, \lambda) \models$ every set of reals is Suslin. By 3.0.1, if all the initial segment based fragments of $\Sigma$ were in $D\left(\mathcal{M}_{\infty}^{I}, \lambda_{\infty}^{I}\right)$, they would be Wadge cofinal in $\operatorname{Hom}_{I}^{*}$, which would imply that the Wadge ordinal of $\mathrm{Hom}_{I}^{*}$ has $V$-cofinality $\omega$. In fact, the $V$-cofinality of this ordinal is often uncountable, as for example in the case of 2.11(c). 
Given that for any $\mathbb{R}$-genericity iteration $I, \operatorname{Hom}_{I}^{*}$ is in $V$, it is natural to conjecture that in fact Hom $_{I}^{*}$ is independent of $I$. We do not see how to prove that in full generality, but we can get reasonably close.

There is a natural partial order for producing $\mathbb{R}$-genericity iterations of $\mathcal{M}$.

Definition 3.2 Let $\mathcal{M}$ be a countable mouse, and $\mathcal{M} \models \lambda$ is a limit of Woodin cardinals. Let $\Sigma$ be an $\omega_{1}+1$ iteration strategy for $\mathcal{M}$. We let $\mathcal{I}(\mathcal{M}, \lambda, \Sigma)$ be the set of all finite sequences

$$
p=\left\langle\mathcal{T}_{0}, \ldots, \mathcal{T}_{n}\right\rangle
$$

such that

(a) $\mathcal{T}_{0}$ is an iteration tree on $\mathcal{M}$, and for $0<k \leq n, \mathcal{T}_{k}$ is an iteration tree on the last model of $\mathcal{T}_{k-1}$, and $\mathcal{T}_{n}$ has a last model, and

(b) the composition $\oplus_{k \leq n} \mathcal{T}_{k}$ is a normal tree on $\mathcal{M}$ played according to $\Sigma$, with empty drop-set, and

(c) letting $i: \mathcal{M} \rightarrow \mathcal{N}$ be the canonical embedding of $\mathcal{M}$ into the last model of $\mathcal{T}_{n}$, and $\delta$ the sup of the lengths of the extenders used in $\oplus_{k \leq n} \mathcal{T}_{k}$, we have $\delta<i(\lambda)$.

We regard $\mathcal{I}$ as a partial order, under reverse inclusion.

Clause (c) of 3.2 guarantees that every $p \in \mathcal{I}$ has a proper extension in $\mathcal{I}$. It is not hard to see that if $G$ is $V$-generic over $\mathcal{I}(\mathcal{M}, \lambda, \Sigma)$, then $I=\bigcup G$ is an $\mathbb{R}$-genericity iteration of $\mathcal{M}$. We call such an $I$ an $\mathcal{I}(\mathcal{M}, \lambda, \Sigma)$-generic ( $\mathbb{R}$-genericity) iteration. We are interested in the case $\mathcal{M}$ is $\omega$-sound and projects to $\omega$, so that $\Sigma$ is determined by $\mathcal{M}$, and we can write $\mathcal{I}(\mathcal{M}, \lambda)$ for $\mathcal{I}(\mathcal{M}, \lambda, \Sigma)$.

If $p=\left\langle\mathcal{T}_{0}, \ldots, \mathcal{T}_{n}\right\rangle \in \mathcal{I}(\mathcal{M}, \lambda, \Sigma)$, then we shall write $\mathcal{T}_{i}(p)=\mathcal{T}_{i}, \mathcal{M}_{0}(p)=\mathcal{M}$ and $\mathcal{M}_{i+1}(p)=$ the last model of $\mathcal{T}_{i}, \mathcal{M}_{p}=\mathcal{M}_{n+1}(p), i_{k, l}^{p}$ for the canonical embedding of $\mathcal{M}_{k}(p)$ into $\mathcal{M}_{l}(p), \lambda_{p}$ for $i_{0, n+1}^{p}(\lambda)$, and $\delta_{p}$ for the sup of the lengths of the extenders used in the $\mathcal{T}_{k}$, for $k \leq n$.

We shall show that for certain natural $(\mathcal{M}, \lambda)$, any two $\mathcal{I}(\mathcal{M}, \lambda)$-generic iterations give rise to the same derived model. The $\mathcal{M}$ we have in mind here are mice like $M_{\omega}^{\sharp}$ (the sharp of the minimal model with $\omega$ Woodins), $M_{\text {adr }}^{\sharp}$ ( the sharp of the minimal model of the $A_{\mathbb{R}}$ hypothesis), and $M_{\mathrm{wlim}}^{\sharp}$ (the sharp of the minimal model with a Woodin limit of Woodins). The main feature of these mice which lets our argument go through is that they reconstruct themselves below the relevant $\lambda$. There are some other conditions we seem to need as well. We are by no means sure that the definitions we are about to give abstract the most general hypotheses on $\mathcal{M}$ and $\lambda$ yielding derived-model invariance.

Definition 3.3 We call a premouse $\mathcal{M}$ a sharp mouse iff 
(a) $\mathcal{M}$ is active, and for some $\alpha<\operatorname{crit}\left(\dot{F}^{\mathcal{M}}\right), \dot{E}_{\eta}^{\mathcal{M}}=\emptyset$ for all $\eta \geq \alpha$,

(b) there is a sentence $\varphi$ such that for $\kappa=\operatorname{crit}\left(\dot{F}^{\mathcal{M}}\right), \mathcal{M} \mid \kappa \models \varphi$, but whenever $\mu=$ $\operatorname{crit}\left(\dot{E}_{\eta}^{\mathcal{M}}\right)$ for some $\eta$, then $\mathcal{M} \mid \mu \not \models \varphi$, and

(c) $\mathcal{M}$ is $\omega_{1}+1$-iterable.

If $\alpha$ is as in part (a), then (a) says that $\mathcal{M}$ is essentially the sharp of $\mathcal{M} \mid \alpha$. Part (b) is a $\varphi$-minimality condition, and it implies that $\mathcal{M}$ projects to $\omega$. If $\mathcal{M}$ is also $\omega$-sound, the iteration strategy asserted to exist in (c) is unique.

The reduct of a sharp mouse $\mathcal{M}$ is $\mathcal{M} \| o(\mathcal{M})$, that is, $\mathcal{M}$ with its last extender removed.

Definition 3.4 Let $\mathcal{M}$ be an $\omega$-sound sharp mouse, and $\lambda$ a limit cardinal in $\mathcal{M}$. We say that $\mathcal{M}$ reconstructs itself below $\lambda$ iff whenever $\mathcal{N}$ is a countable, non-dropping iterate of $\mathcal{M}$ by its unique $\omega_{1}+1$ iteration strategy, with $\lambda^{*}$ the image of $\lambda$, and $\kappa<\lambda^{*}$, and $\mathcal{P}$ is the extender model to which the maximal plus-1 certified ms-array over $\kappa$ of $\mathcal{N}$ converges ( see [21]), then there is a sharp mouse $\mathcal{Q}$ whose reduct is $\mathcal{P}$, and a $\Sigma_{1}$-elementary $\pi: \mathcal{M} \rightarrow \mathcal{Q}$ such that $\pi(\lambda)=\lambda^{*}$.

The maximal plus-1 certified ms-array of $\mathcal{N}$ over $\kappa$ is the output of a $K^{c}$-construction which uses all possible reasonably strong extenders from the $\mathcal{N}$-sequence with critical point $>\kappa$ as background extenders; see [21].

Definition 3.5 A tractable pair is a pair $\langle\mathcal{M}, \lambda\rangle$ such that

(a) $\mathcal{M}$ is an w-sound sharp mouse,

(b) $\mathcal{M} \models " \lambda$ is a limit of Woodin cardinals, and $\operatorname{cof}(\lambda)$ is not measurable", and

(c) $\mathcal{M}$ reconstructs itself below $\lambda$.

Some (probable) examples of tractable pairs are

(1) $M_{\omega}^{\sharp}$, with $\lambda$ the sup of its Woodin cardinals,

(2) $M_{\text {adr }}^{\sharp}$, with $\lambda$ the sup of its Woodin cardinals, or with $\lambda$ the sup of the first $\omega$ Woodin cardinals of $M_{\text {adr }}^{\sharp}$,

(3) $M_{\mathrm{dc}}^{\sharp}$, the sharp of the minimal model with a cardinal $\mu$ which is a limit of Woodins, and such that the set of $\kappa<\mu$ which are $<\mu$-strong has order type $\mu$, with $\lambda=\mu$, or with $\lambda$ the sup of the first $\omega$ Woodins, 
(4) $M_{\text {inlim }}^{\sharp}$, the sharp of the minimal model with a cardinal which is inaccessible, and a limit of Woodins, and a limit of $<\lambda$-strong cardinals, with $\lambda$ the sup of all its Woodins, or with $\lambda$ the sup of its first $\omega$ Woodins,

(5) $M_{\text {wlim }}^{\sharp}$, with $\lambda$ the Woodin limit, or with $\lambda$ the sup of the first $\omega$ Woodins.

The author believes that tractability can be verified in each case using [21]. This is easy to see, except in (2)-(5) with $\lambda$ the sup of the first $\omega$ Woodin cardinals of the mouse in question. The author has not gone through all the details in those cases.

We believe that $M_{\mathrm{adr}}^{\sharp}$ is the least mouse with a derived model satisfying $\mathrm{AD}_{\mathbb{R}}$, and $M_{\mathrm{dc}}^{\sharp}$ is the least mouse with a derived model satisfying $A D_{\mathbb{R}}$ plus $D C$ (or equivalently, $\theta=\theta_{\omega_{1}}$ ). This should come out of Woodin's proof that $A D_{\mathbb{R}}$ is equiconsistent with the $A D_{\mathbb{R}}$ hypothesis, and his parallel result for $A D_{\mathbb{R}}+D C$ versus the $A D_{\mathbb{R}}+D C$ hypothesis, but we have not gone through the details. We shall show in section 8 that the derived model of $M_{\text {wlim }}^{\sharp}$ at its top Woodin satisfies $A D_{\mathbb{R}}$ plus " $\theta=\theta_{\theta}$ ".

Theorem 3.6 Let $\langle\mathcal{M}, \lambda\rangle$ be a tractable pair, and let $I$ and $J$ be $\mathcal{I}(\mathcal{M}, \lambda)$ - generic iterations; then $D\left(\mathcal{M}_{\infty}^{I}, \lambda_{\infty}^{I}\right)=D\left(\mathcal{M}_{\infty}^{J}, \lambda_{\infty}^{J}\right)$.

Proof. Fix $\mathcal{M}$ and $\lambda$, and let $\Sigma$ be the unique $\omega_{1}+1$ iteration strategy for $\mathcal{M}$. Let $\mathcal{I}=$ $\mathcal{I}(\mathcal{M}, \lambda)$.

Given $A \subset \mathbb{R}$, let us say $(p, g)$ captures $A$ iff $p \in \mathcal{I}$, and $g$ is $\mathcal{M}_{p}$-generic over $\operatorname{Col}\left(\omega, \delta_{p}\right)$, and there are trees $S, T \in \mathcal{M}_{p}[g]$ such that

$$
\mathcal{M}_{p}[g] \models S, T \text { are }<\lambda_{p} \text {-absolute complements, }
$$

and

$$
A=\bigcup\left\{p\left[i^{\mathcal{U}}(S)\right] \mid p^{\frown}\langle\mathcal{U}\rangle \in \mathcal{I}\right\}
$$

Here $i^{\mathcal{U}}$ is the embedding along the main branch of $\mathcal{U}$. By the proof of 3.0.1, it is enough to prove

Lemma 3.7 Suppose $(p, g)$ captures $A$; then there are densely many $r \in \mathcal{I}$ such that for some $h,(r, h)$ captures $A$.

Proof. Let $(p, g)$ capture $A$, and let $q \in \mathcal{I}$. We seek an $r \leq q$ and $h$ such that $(r, h)$ captures $A$. The idea is just to reconstruct inside $\mathcal{M}_{q}$, above $\delta_{q}$, a model $\mathcal{R}$ into which $\mathcal{M}$ embeds. We can then iterate $\mathcal{M}_{q}$ above $\delta_{q}$ so that we get an $r \leq q$, with $\mathcal{M}_{p}$ embedding into the image $\mathcal{S}$ of $\mathcal{R}$ under this iteration. Our universally Baire representation of $A$ over $\mathcal{M}_{p}$ then lifts to $\mathcal{S}$, and then to the background universe $\mathcal{M}_{r}$ for $\mathcal{S}$, as desired. 
In order to lift the universally Baire representations, we must work with their associated extender normal forms. Recall that an extender normal form is a map

$$
\mathcal{E}=\left(s \mapsto \mathcal{E}_{s}\right) \text {, for } s \in \omega^{<\omega},
$$

where each $\mathcal{E}_{s}$ is an alternating chain with $2 \cdot \operatorname{dom}(s)$ many models, and $\mathcal{E}_{s}$ extends $\mathcal{E}_{t}$ whenever $s$ extends $t$. For $x \in \omega^{\omega}$, we write $\mathcal{E}_{x}^{e}$ and $\mathcal{E}_{x}^{o}$ for the direct limits along the even and odd branches of the infinite alternating chain associated to $x$. If $\mathcal{E}$ is an extender normal form which uses only extenders with critical point $>\kappa$, and whenever $G$ is $V$-generic for a poset of size $<\kappa$,

$$
\left.V[G] \models \forall x \text { ( exactly one of } \mathcal{E}_{x}^{e} \text { and } \mathcal{E}_{x}^{o} \text { is wellfounded, }\right)
$$

then we call $\mathcal{E}$ a $\kappa$-ENF. We say that $\mathcal{E}$ represents $C$ iff $C \subseteq \mathbb{R}$, and for all $x \in \mathbb{R}$,

$$
x \in C \Leftrightarrow \mathcal{E}_{x}^{e} \text { is wellfounded . }
$$

It is worth noting the following absoluteness property of such representations: if $S, T$ are $\kappa$-absolute complements, and $\mathcal{E}$ is a $\kappa$-ENF which represents $p[S]$ in $V$, then whenever $G$ is $V$-generic for a poset of size $\leq \kappa$, we have that $\mathcal{E}$ represents $p[S]$ in $V[G]$. (This uses the uniqueness of wellfounded branches in $V[G]$ which we have built into our definition of $\kappa$-ENF.)

We say that $\mathcal{E}$ is an $\mathcal{N}$-based $\kappa$-ENF in $\mathcal{N}[g]$ iff $\mathcal{N}$ is a premouse, and $g$ is $\mathcal{N}$-generic over some poset of size $<\kappa$ in $\mathcal{N}$, and $\mathcal{N}[g] \models \mathcal{E}$ is a $\kappa$-ENF, and $\mathcal{E}$ uses only extenders from the sequence of $\mathcal{N}$ and its images whose sup-of-generators is a cardinal in the model in which they appear. The following carries over a basic fact about ENF's to this fine-structural setting.

Sublemma 3.7.1 [[25],[10]] Let $\mathcal{N}$ be a premouse, and $\mathcal{N} \models Z F C+" ~ \lambda$ is a limit of cardinals which are Woodin via extenders on my sequence". Let $g$ be $\mathcal{N}$-generic over some poset of size $<\lambda$; then working inside $\mathcal{N}[g]$, the following are equivalent, for any set $C$ of reals:

(a) $C$ is $<\lambda$-universally Baire,

(b) for any $\kappa<\lambda$ there is an $\mathcal{N}$-based $\kappa$-ENF $\mathcal{E}$ which represents $C$.

We continue with the proof of 3.7. By the sublemma, $\mathcal{M}_{p}[g]$ satisfies the statement that there is a sequence $\left\langle\mathcal{E}(\kappa) \mid \kappa<\lambda_{p}\right\rangle$ such that for each $\kappa, \mathcal{E}(\kappa)$ is an $\mathcal{M}_{p}$-based $\kappa$-ENF representing $p[S]$. Fix such an $\left\langle\mathcal{E}(\kappa) \mid \kappa<\lambda_{p}\right\rangle$. Using a $g$-name for this sequence, we can fix a sequence $\left\langle X_{\kappa} \mid \kappa<\lambda_{p}\right\rangle \in \mathcal{M}_{p}$ such that for all $\kappa<\lambda_{p}$,

(a) $\mathcal{M}_{p} \models\left|X_{\kappa}\right| \leq \delta_{p}$, and 
(b) for all $s \in \omega^{<\omega}, \mathcal{E}_{s}(\kappa) \in X_{\kappa}$.

Now let $\mathcal{N}_{q}$ be the version of $\mathcal{M}$ reconstructed inside $\mathcal{M}_{q}$, with all critical points above $\delta_{q}$. Thus we have a $\Sigma_{1}$ elementary

$$
\pi: \mathcal{M} \rightarrow \mathcal{N}_{q} \subseteq \mathcal{M}_{q}
$$

such that $\pi\left(\lambda_{p}\right)=\lambda_{q}$. Using the fact that $\lambda$ does not have measurable cofinality, and a simple comparison argument, one can show

$$
\operatorname{ran}(\pi) \text { is cofinal in } \lambda_{q} .
$$

This was the reason for our restriction on the cofinality of $\lambda$. We leave the details to the reader.

We can use $\pi$ to lift the iteration from $\mathcal{M}$ to $\mathcal{M}_{p}$ to an iteration tree on $\mathcal{N}_{q}$, and thence via the background-extender iterability proof to an iteration tree on $\mathcal{M}_{q}$ which is above $\delta_{q}$. This gives us a condition $t \leq q$ in $\mathcal{I}$, and a $\Sigma_{1}$ elementary

$$
\sigma: \mathcal{M}_{p} \rightarrow \mathcal{N}_{t} \subseteq \mathcal{M}_{t}
$$

where $\mathcal{N}_{t}$ is a sharp mouse whose reduct is the limit model of a plus-1 certified ms-array in the sense of $\mathcal{M}_{t}$.

Let $h_{0}$ be $\mathcal{M}_{t}$-generic over $\operatorname{Col}\left(\omega, \sigma\left(\delta_{p}\right)\right)$. Letting

$$
Y=\sigma\left(\left\langle X_{\kappa} \mid \kappa<\lambda_{p}\right\rangle\right),
$$

we have that $Y_{\mu}$ is countable in $\mathcal{M}_{t}\left[h_{0}\right]$ for all $\mu<\lambda_{t}$, and $Y_{\sigma(\mu)}$ covers $\sigma^{\text {" }} X_{\mu}$, for all $\mu<\lambda_{p}$. Fix a sequence $\left\langle f_{\mu} \mid \mu<\lambda_{t}\right\rangle \mathrm{n} \mathcal{M}_{t}\left[h_{0}\right]$ such that $f_{\mu}$ maps $\omega$ onto $Y_{\mu}$, for all $\mu$. Finally, fix a real $z$ such that for all $\mu<\lambda_{p}$, the map

$$
s \mapsto f_{\sigma(\mu)}^{-1}\left(\sigma\left(\mathcal{E}_{s}(\mu)\right),\right.
$$

which is essentially a real, is recursive in $z$.

Now let $\delta$ be a Woodin cardinal of $\mathcal{M}_{t}\left[h_{0}\right]$ such that $\sup \left(\sigma\left(\delta_{p}\right), \delta_{t}\right)<\delta<\lambda_{t}$. We do a genericity iteration of $\mathcal{M}_{t}\left[h_{0}\right]$ via an $\mathcal{M}_{t}$-based tree which is above $\sup \left(\sigma\left(\delta_{p}\right), \delta_{t}\right)$, and below $\delta$, and obtain thereby a condition $r \leq t$ in $\mathcal{I}$, and a generic $h$ on $\operatorname{Col}\left(\omega, \delta_{r}\right)$, so that $h_{0}, z \in \mathcal{M}_{r}[h]$. Let

$$
\tau: \mathcal{M}_{t} \rightarrow \mathcal{M}_{r}
$$

be the natural map, and let

$$
\psi=\tau \circ \sigma: \mathcal{M}_{p} \rightarrow \mathcal{N}_{r}
$$

where $\mathcal{N}_{r}=\tau\left(\mathcal{N}_{t}\right)$.

Claim. $(r, h)$ captures $A$.

Proof. Recall that there is a $\nu<\lambda_{r}$ such that in $\mathcal{M}_{r}\left[h_{0}\right]$, any $\nu$-homogeneous set of reals is $<\lambda_{r}$-universally Baire. Fix such a $\nu=\nu_{0}$. Now pick $\mu<\lambda_{p}$ such that 
(a) $\nu_{0}<\psi(\mu)$

(b) $\gamma_{0}<\mu$, where $\gamma_{0}$ is the least Woodin cardinal $\gamma$ of $\mathcal{M}_{p}$ such that $\delta_{r}<\psi(\gamma)$, and

(c) there is a Woodin cardinal $\kappa_{0}$ of $\mathcal{M}_{r}$ such that $\psi\left(\gamma_{0}\right)<\kappa_{0}<\psi(\mu)$.

Since $\lambda_{r}$ is a limit of Woodin cardinals in $\mathcal{M}_{r}$, and $\operatorname{ran}(\psi)$ is cofinal in $\lambda_{r}$, we can easily find such a $\mu, \gamma_{0}$, and $\kappa_{0}$.

Let

$$
\mathcal{E}_{s}=\mathcal{E}_{s}(\mu)
$$

and

$$
\mathcal{F}_{s}=\psi\left(\mathcal{E}_{s}\right)
$$

and

$$
\mathcal{F}_{s}^{*}=\text { alternating chain on } \mathcal{M}_{r}[h] \text { induced by } \mathcal{F}_{s},
$$

for all $s \in \omega^{<\omega}$. Here we mean that $\mathcal{F}_{s}^{*}$ arises from $\mathcal{F}_{s}$ as in the iterability proof, using the background extenders provided by the $K^{c}$-construction in $\mathcal{M}_{r}$ whose output is $\mathcal{N}_{r}$.

We need the following small extension of the theorem 3.3 of [21] on UBH in extender models. We omit the proof.

Lemma 3.8 Let $\mathcal{P} \models$ ZFC be fully iterable mouse, and let $G$ be generic over $\mathcal{P}$ for a poset of size $<\kappa$ in $\mathcal{P}$; then there are no $\mathcal{T}, b, c \in \mathcal{P}[G]$ such that

$$
\mathcal{P}[G] \models \mathcal{T} \text { is a } \mathcal{P} \text {-based plus-2 iteration tree on } \mathcal{P}[G] \text { of limit length, }
$$

with all critical points of extenders in $\mathcal{T}$ above $\kappa$, and $D^{\mathcal{T}}=\emptyset$, and

$$
\mathcal{P}[G] \models b \text { and } c \text { are distinct, cofinal, wellfounded branches of } \mathcal{T} \text {. }
$$

It follows immediately from lemma 3.8 that $\mathcal{M}_{r}[h] \models \mathcal{F}$ is a $\psi(\mu)$-ENF. We therefore have trees $U, W \in \mathcal{M}_{r}[h]$ such that

$$
\mathcal{M}_{r}[h] \models U, W \text { are }<\lambda_{r} \text {-absolute complements, }
$$

and

$$
\mathcal{M}_{r}[h] \models p[U]=\left\{x \mid\left(\mathcal{F}^{*}\right)_{x}^{e} \text { is wellfounded }\right\} .
$$

We claim that $U, W$ witness that $(r, h)$ captures $A$. For that, fix $x \in \mathbb{R}$. It is enough to show that if $x \in A$, then there is a condition $u \leq r$ such that $x \in p\left[i_{r, u}(U)\right]$, and if $x \notin A$, then there is a condition $u \leq r$ such that $x \in p\left[i_{r, u}(W)\right]$. So assume $x \in A$; the proof when $x \notin A$ is completely parallel.

Let $a \leq p$ come from iterating $\mathcal{M}_{p}$ above $\delta_{p}$, and below $\gamma_{0}$, so as to make $x$ generic over $\mathcal{M}_{a}[g]$ for the image of the extender algebra at $\gamma_{0}$. We have then that $x \in p\left[i_{p, a}(S)\right]$, and 
thus working in $\mathcal{M}_{a}[g][x]$, we can conclude that $i_{p, a}(\mathcal{E})_{x}^{o}$ is illfounded. Let $b \leq r$ come from lifting the genericity iteration from $\mathcal{M}_{p}$ to $\mathcal{N}_{r}$ using $\psi$, then further lifting it to $\mathcal{M}_{r}$ using the iterability proof for background-certified models. It is important here to note that the iteration strategy $\Sigma$ on normal extensions of $\mathcal{T}_{p}$ is unique, so that it agrees with the strategy of lifting to $\mathcal{M}_{r}$ as above, and using $\Sigma$ to pick branches of the evolving tree on $\mathcal{M}_{r}$. This shows that the lifting process succeeds, and does give us a condition in $b \in \mathcal{I}$.

Let $\phi: \mathcal{M}_{a} \rightarrow \mathcal{N}_{b}$ be the natural lifting map, where $\mathcal{N}_{b}=i_{r, b}\left(\mathcal{N}_{r}\right)$. We have from the commutativity of the copy maps that

$$
\phi \circ i_{p, a}=i_{r, b} \circ \psi
$$

and from this we get that

$$
\phi\left(i_{p, a}(\mathcal{E})\right)=i_{r, b}(\mathcal{F}) .
$$

Here we understand the identity just displayed by letting the embeddings act on $\mathcal{E}$ "pointwise", for $\phi$ is not actually defined on $\mathcal{M}_{a}[g]$. (This was the reason we moved to extender normal forms in the first place.) We have then that

$$
\left(i_{r, b}\left(\mathcal{F}^{*}\right)\right)_{x}^{o} \text { is illfounded. }
$$

Finally, let $u \leq b$ come from a genericity iteration of $\mathcal{M}_{b}[h]$ above $\delta_{b}$ and below $i_{r, b}\left(\kappa_{0}\right)$ which makes $x$ generic over $\mathcal{M}_{u}[h]$ for the extender algebra at $i_{r, u}\left(\kappa_{0}\right)$. Clearly, we still have

$$
\left(i_{r, u}\left(\mathcal{F}^{*}\right)\right)_{x}^{0} \text { is illfounded. }
$$

But note that $x$ is generic over $\mathcal{M}_{u}[h]$ for a poset of size $<i_{r, u}(\psi(\mu))$, and that the universally Baire representation $i_{r, u}(U)$ is satisfied to be equivalent to $i_{r, u}(\mathcal{F})$ in $\mathcal{M}_{u}[h]$, and that this equivalence is absolute for forcing of size $<i_{r, u}(\psi(\mu))$ over $\mathcal{M}_{u}[h]$. It follows that $x \in$ $p\left[i_{r, u}(U)\right]$, as desired.

This completes the proof of the claim, and hence the proof of lemma 3.7, and hence the proof of theorem 3.6.

\section{Mouse operators and jump operators}

The sharp mice we introduced earlier all relativise: e.g., we can form $M_{\text {adr }}^{\sharp}(y)$ for any transitive set $y$. In this way, we obtain a mouse operator. In this section we give a general notion of mouse operator which is useful in the AD context, and derive some elementary consequences of AD concerning mouse operators. We then record some special properties of the operators one gets by relativising sharp mice.

Definition 4.1 A premouse jump operator is a function $x \mapsto \mathcal{M}(x)$, defined on a Turing cone of reals $x$, such that for each $x$ in its domain 
(1) $\mathcal{M}(x)$ is a $\omega$-sound ms-premouse over $x$ which projects to $\omega$, and

(2) for all $y \equiv_{T} x, \mathcal{M}(y)$ is the canonical re-arrangement of $\mathcal{M}(x)$ as a premouse over $y$.

A mouse jump operator is a premouse jump operator $\mathcal{M}$ such that $\mathcal{M}(x)$ is $\omega_{1}$-iterable, for all $x \in \operatorname{dom}(\mathcal{M})$.

Identifying $\mathcal{M}(x)$ with the real $\operatorname{Th}^{\mathcal{M}(x)}(x)$, we see that any premouse jump operator is uniformly Turing-invariant, and hence a jump operator in the sense of [28]. We shall use the notation and results of that paper. In particular, $\leq_{m}$ is the prewellorder of jump operators defined by Martin.

The next lemma is implicit in [28].

Lemma 4.2 Assume $\mathrm{AD}^{+}$and $\mathrm{MSC1}$, and let $J$ be a jump operator; then

(1) there is a premouse jump operator $\mathcal{N}$ such that $J \equiv_{m} \mathcal{N}$, and

(2) if $J$ is $\boldsymbol{\Delta}_{1}^{2}$, then there is a mouse jump operator $\mathcal{N}$ such that $J \equiv_{m} \mathcal{N}$.

Proof. We first prove (2). Let $J$ be $\Delta_{1}^{2}(z)$ and its domain contain the cone above $z$. For $x \geq_{T} z$, let

$$
\mathcal{P}(x)=\text { least } x \text {-mouse } \mathcal{M} \text { such that } J(x) \in \mathcal{M} \text {. }
$$

Since $J(x)$ is $\mathrm{OD}(x)$, there is such an $x$-mouse by MSC1. Identifying $\mathcal{P}(x)$ with its theory, it is easy to see that $\mathcal{P}$ is a mouse jump operator, and $J \leq_{m} \mathcal{P}$.

It follows from the proof of Theorem 3 of [28] that if $\mathcal{Q}$ is a mouse jump operator, and $H \leq_{m} \mathcal{Q}$, then there is a mouse jump operator $\mathcal{N}$ such that $J \equiv_{m} \mathcal{N}$. (This was observed by M. Rudominer and the author.) This proves (2).

Part (1) follows from part (2) and Woodin's basis theorem for $\Sigma_{1}^{2}$ (see 9.10, part (b)). For notice that it is a projective statement about $J$ to say that it is a counterexample to (1). Thus if there is any counterexample $J$ to (1), there is a $\Delta_{1}^{2}$ counterexample $J$ to (1). But then $J$ is a counterexample to (2).

A minor variant of a proposition from [18] shows that every mouse jump operator is preserved by Cohen forcing on a cone:

Lemma 4.3 Assume $\mathrm{AD}$, and let $\mathcal{M}$ be a mouse jump operator; then for a cone of reals $r$, we have that whenever $g$ is Cohen generic over $\mathcal{M}(r)$, then $\mathcal{M}(r)[g]$, when regarded as a mouse over $\langle r, g\rangle$, is equal to $\mathcal{M}(\langle r, g\rangle)$. 
Proof. Let $f(r)=o(\mathcal{M}(r))$. $f$ is a Turing invariant, ordinal-valued function. It is easy to see that any such function is order-preserving on a cone, so that for a cone of $r, f(r) \leq f(s)$ whenever $r \leq_{T} s$. Since there is at most one sound $\langle r, g\rangle$-mouse projecting to $\omega$ of a given height, it is enough to see that for a cone of $r, f(r)=f(\langle r, g\rangle)$ whenever $g$ is Cohen generic over $\mathcal{M}(r)$. Suppose not, and let $r_{0}$ be the base of a cone where $f(r)<f(\langle r, g\rangle)$ holds. Let $g:(\omega \times \omega) \rightarrow 2$ be Cohen generic with respect to all $\mathrm{OD}\left(r_{0}\right)$ dense sets, and let $r_{i}=\left\langle r_{0}, g \uparrow(\omega \backslash i) \times \omega\right.$. Clearly, $r_{i} \equiv_{T}\left\langle r_{i+1}, g\right\rangle$. for some $g$ Cohen over $\mathrm{OD}\left(r_{i+1}\right)$. But $\mathcal{M}\left(r_{i+1}\right) \subseteq \mathrm{OD}\left(r_{i+1}\right)$, so $f\left(r_{i+1}\right)<f\left(r_{i}\right)$ for all $i$, a contradiction.

We shall show that every mouse jump operator has a canonical extension to a operator defined on a cone of countable transitive sets, and take mouse operators to just be these canonical extensions. The extension is obtained simply by looking at generic enumerations of the countable transitive set.

Definition 4.4 An HC-cone is a set of the form $c=\left\{b \mid b\right.$ is countable and transitive, and $V_{\omega} \cup$ $\{x\} \subseteq b\}$, where $x$ is a real. We say in this case that $x$ is a base for the cone $c$.

Theorem 4.5 Assume AD, and let $\mathcal{M}_{0}$ be a mouse jump operator which is defined, and preserved by Cohen forcing in the sense of 4.3, on the Turing cone above $x$; then for each a in the $H C$-cone with base $x$, there is a unique $\omega$-sound mouse $\mathcal{P}$ over a such that such that whenever $g$ is $\operatorname{Col}(\omega, a)$-generic over $\mathcal{P}$, and $r_{g}$ is some real coding $\langle a, g\rangle$ in a natural way (so that $r_{0} \leq_{T} r$ ), we have

$$
\mathcal{P}[g]=\mathcal{M}_{0}\left(r_{g}\right)
$$

Proof. (Sketch.) Fix $g$ which is $\mathrm{OD}(a)$-generic over $\operatorname{Col}(\omega, a)$. It is easy to construct by induction on levels a mouse $\mathcal{P}^{g}$ over a such that $\mathcal{P}^{g}[g]=\mathcal{M}_{0}\left(r_{g}\right)$, by simply restricting the extender sequence of $\mathcal{M}_{0}\left(r_{g}\right)$ to the model one is building. See [24] or [15] for the details of this construction. But if $g$ and $h$ agree at all but finitely many arguments, we have $r_{g} \equiv_{T} r_{h}$, so $\mathcal{M}_{0}\left(r_{g}\right)$ is $\mathcal{M}_{0}\left(r_{h}\right)$ up to a trivial re-arrangement, which implies $\mathcal{P}^{g}=\mathcal{P}^{h}$. It follows that $\mathcal{P}^{g}$ is constant on a comeager set of $g$, and we let $\mathcal{P}$ be this constant value. We then have that $\mathcal{P}$ is a sound mouse over $a$ projecting to $a$, and for comeager many $g$ on $\operatorname{Col}(\omega, a), \mathcal{P}[g]$ is $\mathcal{M}_{0}\left(r_{g}\right)$, when re-arranged as a mouse over $r_{g}$.

Now let $h$ be an arbitrary $\operatorname{Col}(\omega, a)$-generic over $\mathcal{P}$. Let $g$ be $\operatorname{Col}(\omega, a)$-generic over $\mathrm{OD}\left(r_{h}\right)$, and in the comeager set where $\mathcal{P}[g]=\mathcal{M}_{0}\left(r_{g}\right)$ (re-arranged). Let $\mathcal{Q}\left(r_{h}\right)$ be the re-arrangement of $\mathcal{P}[h]$ as a mouse over $r_{h}$. We have

$$
\mathcal{Q}\left(r_{h}\right)[g]=\mathcal{P}[h][g]=\mathcal{P}[g][h]=\mathcal{M}_{0}\left(r_{g}\right)[h]=\mathcal{M}_{0}\left(\left\langle r_{g}, r_{h}\right\rangle\right)
$$

using 4.3 for the last equality. So

$$
\mathcal{Q}\left(r_{h}\right)[g]=\mathcal{M}_{0}\left(r_{h}\right)[g]
$$


by 4.3, which then implies $\mathcal{Q}\left(r_{h}\right)=\mathcal{M}_{0}\left(r_{h}\right)$, because the two are sound mice over $r_{h}$ projecting to $\omega$ and having the same ordinals. This shows there is a mouse $\mathcal{P}$ as claimed. It is unique because it projects to $a$, and is $a$-sound.

It is easy to see that the mouse $\mathcal{P}$ of the claim is unique, so we may set $\mathcal{M}(a)=\mathcal{P}$. We leave it to the reader to check that $\mathcal{M}\left(V_{\omega} \cup\{r\}\right)=\mathcal{M}_{0}(r)$ when $r$ is a real $\geq_{T} r_{0}$. For arbitrary $a \in \mathrm{HC}$, let us write $\mathcal{M}(a)$ for $\mathcal{M}\left(V_{\omega} \cup \mathrm{TC}(a \cup\{a\})\right.$. We have then that $\mathcal{M}$ extends $\mathcal{M}_{0}$.

Definition 4.6 Let $\mathcal{M}_{0}$ be a premouse jump operator defined on all reals $r \geq_{T} r_{0}$. We say $\mathcal{M}_{0}$ is $\mathrm{HC}$-extendible iff for any a in the $H C$-cone with base $r_{0}$, there is an a-premouse $\mathcal{P}$ such that whenever $g$ is $\operatorname{Col}(\omega, a)$-generic over $\mathcal{P}$, and $r_{g}$ is some real coding $\langle a, g\rangle$ in a natural way (so that $r_{0} \leq_{T} r$ ), we have

(1) $\mathcal{P}[g]=\mathcal{M}_{0}\left(r_{g}\right)$, and

(2) $\mathcal{P}$ is obtained from $\mathcal{M}_{0}\left(r_{g}\right)$ via the contruction in the proof of 4.5 .

If $\mathcal{M}_{0}$ is $H C$-extendible, then letting $\mathcal{M}(a)$ be the unique $\mathcal{P}$ as in (1) and (2), we call $\mathcal{M}$ the HC-extension of $\mathcal{M}_{0}$.

We have then by 4.5 that every mouse jump operator is HC-extendible to some HC-cone. This strengthens part (2) of 4.2, and we can use it to strengthen part (1) as well:

Lemma 4.7 Assume $\mathrm{AD}^{+}$and $\mathrm{MSC1}$, and let $J$ be a jump operator; then there is an $\mathrm{HC}$ extendible premouse jump operator $\mathcal{N}$ such that $J \equiv_{m} \mathcal{N}$.

Proof. Again, this follows from Woodin's basis theorem for $\Sigma_{1}^{2}$. If there is any counterexample, there is a $\boldsymbol{\Delta}_{1}^{2}$ counterexample $J$ to (1). But by $4.2(2)$ and 4.5 , there is no $\boldsymbol{\Delta}_{1}^{2}$ counterexample.

Definition 4.8 $\mathcal{M}$ is a premouse operator iff $\mathcal{M}$ is the HC-extension of some premouse jump operator. $\mathcal{M}$ is a mouse operator iff it is the HC-extension of some mouse jump operator.

One useful property a premouse operator might have is condensation.

Definition 4.9 Let $\mathcal{M}$ be a premouse operator defined on the $H C$-cone with base $x$. We say $\mathcal{M}$ condenses well (or has condensation) iff whenever $\pi: \mathcal{P} \rightarrow \mathcal{M}(a)$ is $\Sigma_{1}$ elementary, and $T C(x \cup\{x\}) \subseteq \operatorname{ran}(\pi)$, then $\mathcal{P}=\mathcal{M}\left(\pi^{-1}(a)\right)$.

It is not the case that every mouse operator condenses well on some HC-cone. Operators determined by $\Pi_{2}$ theories do, however. 
Definition $4.10 A \Pi_{2}$ mouse (jump) operator is a mouse (jump) operator $\mathcal{M}$ such that for some set $T$ of $\Pi_{2}$ sentences in the language of relativised premice, for all $x \in \operatorname{dom}(\mathcal{M})$, $\mathcal{M}(x)$ is the least $\omega_{1}+1$-iterable ms-mouse $\mathcal{P}$ over $x$ such that $\mathcal{P} \models T$.

Lemma 4.11 Let $\mathcal{M}_{0}$ be a $\Pi_{2}$ mouse jump operator, and $\mathcal{M}$ its unique $H C$-extension; then $\mathcal{M}$ is a $\Pi_{2}$ mouse operator.

Lemma 4.12 Every $\Pi_{2}$ mouse operator condenses well.

We now turn to the special case of sharp mice.

The sharp mice we introduced earlier all relativise: e.g., we can form $M_{\text {adr }}^{\sharp}(y)$ for any transitive set $y$. Similarly, the $\lambda$ 's which were part of our tractable pairs relativise. We now abstract some properties of the resulting operators:

Definition $4.13 A$ sharp-mouse operator is a mouse operator $\mathcal{M}$ such that there is a sentence $\varphi$ in the language of relativised premice such that for all $y \in \operatorname{dom}(\mathcal{M})$

(a) $\mathcal{M}(y)$ is active, and for some $\alpha<\operatorname{crit}\left(\dot{F}^{\mathcal{M}(y)}\right), \dot{E}_{\eta}^{\mathcal{M}(y)}=\emptyset$ for all $\eta \geq \alpha$,

(b) for $\kappa=\operatorname{crit}\left(\dot{F}^{\mathcal{M}(y)}\right), \mathcal{M}(y) \mid \kappa=\varphi$, but whenever $\mu=\operatorname{crit}\left(\dot{E}_{\eta}^{\mathcal{M}(y)}\right)$ for some $\eta$, then $\mathcal{M}(y) \mid \mu \not \models \varphi$.

Note that if $\mathcal{M}$ is a sharp-mouse operator, then $\mathcal{M}(y)$ is sound and $\omega_{1}+1$-iterable, for all $y \in \operatorname{dom}(\mathcal{M})$.

Definition 4.14 Let $\mathcal{M}$ be an sharp mouse operator, and $\lambda=(y \mapsto \lambda(y))$ be such that for all $y \in H C, \lambda(y)$ is a limit cardinal in $\mathcal{M}(y)$. We say that $\mathcal{M}$ reconstructs itself below $\lambda$ iff whenever $y \in H C$ and $\mathcal{N}(y)$ is a countable, non-dropping iterate of $\mathcal{M}(y)$ by its unique $\omega_{1}+1$ iteration strategy, with $\lambda^{*}$ the image of $\lambda(y)$, and $\kappa<\lambda^{*}$, and $z$ is $\mathcal{N}(y)$-generic over a poset of size $<\kappa$ in $\mathcal{N}(y)$ and $\mathcal{P}(z)$ is the extender model to which the maximal plus-1 certified ms-array over $\kappa$ of $\mathcal{N}(y)[z]$ converges ( see [21]), then there is a sharp mouse $\mathcal{Q}(z)$ whose reduct is $\mathcal{P}(z)$, and a $\Sigma_{1}$-elementary $\pi: \mathcal{M}(z) \rightarrow \mathcal{Q}(z)$ such that $\pi(\lambda(z)) \leq \lambda^{*}$.

Definition 4.15 $A$ tractable operator is a pair $(\mathcal{M}, \lambda)$ such that

(a) $\mathcal{M}$ is a sharp-mouse operator,

(b) $\lambda=(y \mapsto \lambda(y))$ is such that $\lambda(y)$ is definable from $y$ over $\mathcal{M}(y)$, uniformly in $y$,

(c) for all $y, \mathcal{M}(y) \models " \lambda(y)$ is a limit of Woodin cardinals, and $\operatorname{cof}(\lambda(y))$ is not measurable",

(d) $\mathcal{M}$ reconstructs itself below $\lambda$. 
With these definitions, we can extend 3.6 to:

Theorem 4.16 Let $\langle\mathcal{M}, \lambda\rangle$ be a tractable operator, let $x, y \in H C$, and let $I$ and $J$ be $\mathcal{I}(\mathcal{M}(x), \lambda(x))$ and $\mathcal{I}(\mathcal{M}(y), \lambda(y))$ generic iterations; then $D\left(\mathcal{M}(x)_{\infty}^{I}, \lambda_{\infty}^{I}\right)=D\left(\mathcal{M}(y)_{\infty}^{J}, \lambda_{\infty}^{J}\right)$.

Later we shall need a further strengthening of 3.6, in which the relativised mice are hybrid mice of the form $\mathcal{M}^{\Sigma}(y)$, where $\Sigma$ is an appropriate set of reals in the derived model of $\mathcal{M}(y)$.

\section{The mouse set conjecture in $D(\mathcal{M}, \lambda)$}

If $\mathcal{M}$ is a mouse, then $\mathcal{M}$ itself "captures" all reals which are OD in its derived model. So the following result is not surprising:

Theorem 5.1 Let $(\mathcal{M}, \lambda)$ be a tractable operator, and suppose $D$ is a realization of $D(\mathcal{M}(z), \lambda)$, and $y$ is transitive and countable in $D$, and $x \subseteq y$. Then the following are equivalent:

(a) $D \models x \in O D(y \cup\{y\})$,

(b) $x \in \mathcal{M}(y)$,

(c) $D \models x$ is in an $\omega_{1}$-iterable mouse over $y$.

Proof. $(a) \Rightarrow(b)$ : We easily get that $D^{*}=x \in \mathrm{OD}(y \cup\{y\})$, where $D^{*}=D\left(\mathcal{M}(z)_{\infty}^{I}\right)$ for an $\mathcal{I}(\mathcal{M}(z), \lambda(z))$-generic $I$. But then $D^{*}=D\left(\mathcal{M}(y)_{\infty}^{J}\right)$ for an $\mathcal{I}(\mathcal{M}(y), \lambda(y))$-generic $J$. Since the forcing is homogeneous, $x \in \mathcal{M}(y)_{\infty}^{J}$. Therefore $x \in \mathcal{M}(y)$.

$(b) \Rightarrow(c)$ (Sketch): It is enough to show that if $\mathcal{P}$ is a proper initial segment of $\mathcal{M}(y)$, and $\mathcal{P}$ projects to $y$, then $\mathcal{P}$ has an $\omega_{1}$-iteration strategy in $D(\mathcal{M}(y), \lambda)$. (One can then pass to $D=D(\mathcal{M}(z), \lambda(z))$ by 4.16.) For that, we show that the unique strategy $\Sigma$ for $\mathcal{P}$ has a $\mu$-UB code in $\mathcal{M}(y)$, for any $\mu<\lambda(y)$. Fix $\mu<\lambda(y)$. Because $\mathcal{M}(y)$ reconstructs itself below $\lambda(y)$, the full background extender construction $\left\langle\mathcal{C}_{k}\left(\mathcal{N}_{\eta}\right)\right\rangle$ of $\mathcal{M}(y)$, done with all critical points $>\mu$ (and using $\mu$-closed extenders from the sequence of $\mathcal{M}(y)$ as backgrounds), reaches $\mathcal{P}=\mathcal{C}_{k}\left(\mathcal{N}_{\eta}\right)$. Let $\mathcal{M}(y) \mid \xi \models \mathcal{P}=\mathcal{C}_{k}\left(\mathcal{N}_{\eta}\right) \wedge$ ZFC . By the results of section 12 of [12], $\Sigma$ is induced by lifting trees on $\mathcal{P}$ to trees on $\mathcal{M}(y)$, then following the unique strategy for $\mathcal{M}(y)$. The lifting process is uniformly definable over $\mathcal{M}(y)[g]$, for any size $<\mu$ generic $g$ over $\mathcal{M}(y)$, and this definition has the generic absoluteness required to obtain a $\mu$-UB code. The key to the generic absoluteness is the fact that UBH holds in ms-mice, or more precisely, the sharper version of this fact stated as Theorem 3.4 in [21]. The iteration trees on $\mathcal{P}$ being lifted have size less than the closure of the background extenders, so by UBH they are continuously illfounded off the branches they choose, which guarantees both the existence-in- $\mathcal{M}(y)[g]$ and the absolute definabilty of the branches chosen by $\Sigma$. 
[Here is a bit more detail. Let $\varphi$ be the formula which defines $\Sigma$ over $<\mu$-generic extensions of $\mathcal{M}(y) \mid \xi$ via this lifting process. It is enough to show that club many hulls of $\mathcal{M}(y) \mid \xi$ are generically correct. So let $\pi: N \rightarrow \mathcal{M}(y)$ be elementary, where $N$ is transitive, with $\pi \uparrow(\mathcal{P} \cup\{\mathcal{P}\})=$ identity. Let $g$ be $<\pi^{-1}(\mu)$-generic over $N$. Let $\mathcal{T}$ be an iteration tree satisfying $\varphi$ over $N[g]$. We need to see $\mathcal{T}$ is by $\Sigma$. Since $\Sigma$ is the unique strategy for $\mathcal{P}$, it suffices to see that the phalanx $\Phi(\mathcal{T})$ is iterable. Let $\mathcal{U}$ be the lift of $\mathcal{T}$ to $N$, as in [12]. It suffices to see $\Phi(\mathcal{U})$ is iterable. But $\Phi(\mathcal{U})$ is continuously illfounded off the branches it chooses, and therefore must be according to any iteration strategy for $N$.]

$(c) \Rightarrow(b)$ : This is one of the elementary corollaries of the comparison lemma. (Note $\omega_{1}$-iterability implies $\left(\omega_{1}+1\right)$-iterability, granted AD.)

The independence of the derived model $D\left(\mathcal{M}_{\infty}^{I}\right)$ for arbitrary $\mathbb{R}$-genericity iterations (as opposed to $\mathcal{I}$-generic ones) is perhaps not so important. However, 5.1 does give us something there.

Corollary 5.2 Let $(\mathcal{M}, \lambda)$ be a tractable operator, and let $D_{0}$ and $D_{1}$ be derived models associated to $\mathbb{R}$-genericity iterations of $\mathcal{M}(x)$ and $\mathcal{M}(y)$. Then $\left(\Sigma_{1}^{2}\right)^{D_{0}}=\left(\Sigma_{1}^{2}\right)^{D_{1}}$. Thus if $D_{0}=\theta=\theta_{0}$, then $D_{0}=D_{1}$.

The proof is an easy consequence of the fact that, assuming $\mathrm{AD}^{+}, x \in \mathrm{OD}(y)$ is a $\Sigma_{1^{-}}^{2}$ complete relation on reals. (Note also $D_{0} \equiv D_{1}$ by 3.6, and since we are talking about "old" derived models, if $\theta_{0}=\theta$ in such a model, then it is of the form $L(\mathbb{R} \cup\{U\})$, for $U$ a $\Sigma_{1^{-}}^{2}$ complete set.)

We also get a sort of converse to 2.11(a), in the case the ground model is a mouse.

Corollary 5.3 Let $(\mathcal{M}, \lambda)$ be a tractable operator, and suppose that for some $x, \mathcal{M}(x)=$ $\lambda(x)$ is a limit of cutpoints. Then for all $x, \mathcal{M}(x) \models \lambda(x)$ is a limit of cutpoints, moreover and derived model $D(\mathcal{M}(x), \lambda(x))$ satisfies $\theta_{0}=\theta$.

Proof. Let $D$ be a realization of $D(\mathcal{M}(x), \lambda(x))$, ans suppose toward contradiction that $D \models \theta_{0}<\theta . \mathrm{AD}^{+}$and $\theta_{0}<\theta$ together imply that the relation on reals $z \notin \mathrm{OD}(y)$ can be uniformized by a Suslin-co-Suslin function. Let $f(y)=z$ be such a function in the sense of $D$. Because the cutpoints of $\mathcal{M}(x)$ are cofinal below $\lambda(x)$, we can find such a cutpoint $\eta$ and a $g$ which is $\mathcal{M}(x)$-generic for $\operatorname{Col}(\omega, \eta)$ such that $f=p[T]$, for some tree $T$ in $\mathcal{M}(x)[g]$. Let $y$ be a real in $\mathcal{M}(x)[g]$ which codes $\langle\mathcal{M}(x) \mid \eta, g\rangle$ is some simple way, and let $z=f(y)$. Because $T \in \mathcal{M}(x)[g]$, we get $z \in \mathcal{M}(x)[g]$. But $\eta$ is a cutpoint, so $\mathcal{M}[x][g]$ can be re-arranged as a mouse over $y$. It is not hard to see that this mouse must be $\unlhd \mathcal{M}(y)$, so $z \in \mathcal{M}(y)$, so $z \in \mathrm{OD}(y)$ in $D$, a contradiction.

Remark 5.4 The proof of Corollary 5.2 shows a bit more. Let $(\mathcal{M}, \lambda)$ and $\mathcal{M}, \gamma)$ be tractable operators, with the same mouse operator $\mathcal{M}$, but different limits of Woodins below 
which our $\mathbb{R}$-genericity iterations are being done. Let $D_{0}$ and $D_{1}$ be derived models associated to $\mathbb{R}$-genericity iterations of $\mathcal{M}(x)$ below $\lambda(x)$ and $\mathcal{M}(y)$ below $\gamma(y)$. Then $\left(\Sigma_{1}^{2}\right)^{D_{0}}=\left(\Sigma_{1}^{2}\right)^{D_{1}}$. Thus if $D_{0} \models \theta=\theta_{0}$, then $D_{0}$ is a Wadge initial segment of $D_{1}$. This is what happens if $\lambda(x)$ is the sup of the first $\omega$ Woodin cardinals of $\mathcal{M}(x)$, by 5.3. $D_{0}$ is then just $L(U, \mathbb{R})$, where $U$ is the universal $\Sigma_{1}^{2}$ set of $D_{1}$.

\section{The Solovay sequence in $D(\mathcal{M}, \lambda)$}

We are not sure how to properly state or prove the results of this section in the case of arbitrary tractable operators. We can handle the specific operators introduced above: $M_{\text {adr }}^{\sharp}, M_{\mathrm{dc}}^{\sharp}, M_{\text {inlim }}^{\sharp}, M_{\text {wlim }}^{\sharp}$, with $\lambda$ the sup of the Woodin cardinals of the mouse in each case. We can handle other tractable operators like these, but have not abstracted a good definition. So for now, let us call those four operators paradigmatic. Woodin's 2.11 implies that for our paradigmatic $(\mathcal{M}, \lambda)$, the derived model $D(\mathcal{M}, \lambda)$ satisfies $\mathrm{AD}_{\mathbb{R}}$, or equivalently, $\theta=\theta_{\xi}$ for some limit ordinal $\xi$. In fact, one can give a somewhat simpler proof of 2.11 in this case, one which gives more information as to what sets of reals sit at the $\theta_{\alpha}$ 's in the Wadge hierarchy of the derived model. To begin with

Theorem 6.1 Let $(\mathcal{M}, \lambda)$ be paradigmatic, and let $I$ be $\mathcal{I}(\mathcal{M}(x), \lambda(x))$-generic, and $D=$ $D\left(\mathcal{M}_{\infty}^{I}, \lambda_{\infty}^{I}\right)$. Then the function

$$
z \mapsto \mathcal{M}(z), \text { for } z \in \mathbb{R}
$$

is in $D$, and has Wadge rank $\theta_{0}$ in $D$.

Proof. We may as well take $x=\emptyset$. Let us write $\mathcal{M}=\mathcal{M}(\emptyset), \lambda=\lambda(\emptyset)$. Let $\kappa$ be the least $<\lambda$-strong cardinal, and $\delta$ the least Woodin cardinal $>\kappa$, in $\mathcal{M}$. By 3.6, we may assume that the first normal tree in $I$ is a genericity iteration below $\delta$, with all critical points above $\kappa$, giving rise to

$$
i_{0,1}: M_{0}^{I} \rightarrow M_{1}^{I}
$$

in such a way that

$$
\mathcal{M}=M_{0}^{I} \in M_{1}^{I}[g]
$$

with $g$ being $\operatorname{Col}\left(\omega, i_{0,1}(\delta)\right)$-generic over $M_{1}^{I}$. We can also assume that $\operatorname{crit}\left(i_{1, \infty}\right)>i_{0,1}(\delta)$.

Claim 1. The function $z \mapsto \mathcal{M}(z)$, for $z \in \mathrm{HC}^{V}$, is in the symmetric model $M_{\infty}^{I}\left(\mathbb{R}^{V}\right)$.

Proof.

Note that we can extend $i_{1, \infty}$ to act on $M_{1}[g]$. Fix $z \in \mathrm{HC}^{V}$; we show informally how to compute $\mathcal{M}(z)$ inside $M_{\infty}\left(\mathbb{R}^{V}\right)$. First, let $\alpha<\lambda_{\infty}$ be an inaccessible cardinal of $M_{\infty}$ large enough that $z$ is generic over $M_{\infty}[g] \mid \alpha$, and $i_{0,1}(\delta)<\alpha$. Let $E$ be an extender on the $M_{\infty}$ 
sequence such that $\kappa=\operatorname{crit}(E)$ and $\alpha \leq \operatorname{lh}(E)$. In $M_{\infty}[g]$ we have $\mathcal{M}$ as a set, and hence we can form $\operatorname{Ult}(\mathcal{M}, E)$ as a set. Since $\mathcal{M}\left|\kappa^{+\mathcal{M}}=M_{\infty}\right| \kappa^{+M_{\infty}}$, we have that

$$
\operatorname{Ult}(\mathcal{M}, E)\left|i_{E}^{\mathcal{M}}(\kappa)=\operatorname{Ult}\left(M_{\infty}, E\right)\right| i_{E}^{M_{\infty}}(\kappa)
$$

and thus

$$
\operatorname{Ult}(\mathcal{M}, E)\left|\alpha=M_{\infty}\right| \alpha
$$

by coherence. Thus $z$ is generic over $\operatorname{Ult}(\mathcal{M}, E)$ for a partial order of size $<i_{E}(\lambda)$. Since the $(\mathcal{M}, \lambda)$ operator is tractable, we can now use the modified background-extender construction of [21] to re-build $\mathcal{M}(z)$ from $\operatorname{Ult}(\mathcal{M}, E)[z]$. Once again, the key is that we get $\mathcal{M}(z)$ as a set in $M_{\infty}[g][z]$.

Claim 2. The function $F=z \mapsto \mathcal{M}(z)$, for $z \in \mathbb{R}^{V}$, is in $D\left(M_{\infty}, \lambda_{\infty}\right)$; in fact, it has a universally Baire code in $M_{\infty}[g]$.

Proof. Working in $M_{\infty}[g]$, where we have $\mathcal{M}$, let $\alpha$ be any inaccessible cardinal as in claim 1. Let $E$ be an extender on the $M_{\infty}$-sequence such that $\kappa=\operatorname{crit}(E)$ and $\alpha<\operatorname{lh}(E)$. Let $\varphi$ be the formula defining $F$ on size $<\alpha$ generic extensions of $M_{\infty}[g]$ from $\mathcal{M}$ and $E$ which is implicit in the proof of claim 1.

Let $\xi$ be sufficiently large. Since any tractable operator condenses to itself, we get that in $\mathcal{M}_{\infty}[g]$, there are club many countable $X \prec M_{\infty} \mid \xi$ such that if $N$ is the transitive collapse of $X$, and $H$ is $N$-generic for a poset of size $<$ the collapse of $\alpha$, then $\varphi$ defines $F \uparrow N[H]$ over $N[H]$ from $\mathcal{M}$ and the collapse of $E$. (I.e., there are club many generically correct $X$.) As is well known, this gives us an $\alpha$-universally Baire code for $F \uparrow M_{\infty}[g]$ in $M_{\infty}[g]$. It is easy to see that the code $\left(T, T^{*}\right)$ has the property that $p[T] \cap M_{\infty}[g][H]=F \cap M_{\infty}[g][H]$ for all size $<\alpha$ generic $H$.

Claim 3.The function $F=z \mapsto \mathcal{M}(z)$, for $z \in \mathbb{R}^{V}$, has Wadge rank $\theta_{0}$ in $D\left(M_{\infty}, \lambda_{\infty}\right)$.

Proof. Suppose $F$ were $\mathrm{OD}(z)$ in $D\left(M_{\infty}, \lambda_{\infty}\right)$, where $z \in \mathbb{R}$. By 5.1 , we then get $F(z) \in$ $\mathcal{M}(z)$, a contradiction. Thus $F$ has Wadge rank at least $\theta_{0}$.

Let $\mathcal{M}(z)^{-}$be the proper class model obtained by iterating away the last extender of $\mathcal{M}(z)$, and put

$$
F_{n}(z)=\text { type of } 1 \text { st } n \text { indiscernibles over } \mathcal{M}(z)^{-},
$$

for $n<\omega$ and $z \in \mathbb{R}$. It is clear that $F$ is Wadge equivalent to the join of the $F_{n}$ 's. (This is actually just a matter of definition; strictly speaking, $F(z)$ isn't even a real. It is coded by its 1st order theory, however, and this theory is easily intercomputable with the join of the $F_{n}(z)$.) It is enough then to show that each $F_{n}$ is $\mathrm{OD}(\mathbb{R})$ in $D\left(M_{\infty}, \lambda_{\infty}\right)$.

For this, note first that $F_{n}$ is uniformly Turing invariant, that is, it is a jump operator. Also, $F_{n}(z) \in \mathcal{M}(z)$ for all $z$, so we can set

$$
g(z)=\text { least } \alpha \text { such that } F_{n}(z) \in \mathcal{M}(z) \mid \alpha,
$$


and

$$
G(z)=\Sigma_{1} \text { theory of } p \text { in } \mathcal{M}(z) \mid g(z),
$$

where $p$ is the first standard parameter of $\mathcal{M}(z) \mid g(z)$. Clearly $z \equiv_{T} y \Rightarrow g(z)=g(y)$, so $g$ induces a function from the Turing degrees $\mathcal{D}$ to OR, which has an ordinal rank $\gamma$ in $\mathrm{OR}^{\mathcal{D}}$ mod the Martin measure. Also, $G$ is a jump operator, so by the prewellordering of jump operators (see [28]) we have a recursively pointed perfect set $P$ and an $e$ such that $\forall x \in P$, $F_{n}(x)=\{e\}^{G(x)}$. Clearly $\gamma$ and $P$ determine the value of $F_{n}(x)$ on a pointed perfect set's worth of $x$. However, it is then easy to see that this determines the value of $F_{n}(x)$ at all $x$. Thus $F_{n}$ is definable over $D\left(M_{\infty}, \lambda_{\infty}\right)$ from $\gamma$ and $P$.

The claims complete our proof.

Remark 6.2 Something close to 6.1 was first proved by Woodin, by another method.

We can produce mouse operators sitting at the higher $\theta_{\alpha}$ in $D\left(M_{\infty}^{I}, \lambda_{\infty}^{I}\right)$ by nesting the $\mathcal{M}$-operator at various depths. For the mouse operators $\mathcal{M}^{\alpha}$ we define this way, $\mathcal{M}^{\alpha}(x)$ will only be defined on the cone of $x$ for which $\alpha$ is countable in the first admissible set over $x$. This is because we want $\mathcal{M}^{\alpha}(x)$ to project to $x$.

Definition 6.3 Let $\mathcal{M}$ be a premouse operator, and let $\mathcal{P}$ be a premouse, with $\lambda \leq o(\mathcal{P})$. We say that $\mathcal{P}$ is $\mathcal{M}$-closed below $\lambda$ iff whenever $\xi<\lambda$ and $\xi$ is a cutpoint of $\mathcal{P}$, then $\mathcal{M}(\mathcal{P} \mid \xi) \unlhd \mathcal{P}$.

Notice that for each of our paradigmatic operators $(\mathcal{M}, \lambda)$ we have a large cardinal hypothesis $\varphi$ such that $\lambda$ is the first $\eta$ such that the truth of $\varphi$ in $\mathcal{M}$ is witnessed by the total extenders from the $\mathcal{M} \mid \eta$-sequence. Let us call $\varphi$ the hypothesis associated to $(\mathcal{M}, \lambda)$.

Definition 6.4 Let $(\mathcal{M}, \lambda)$ be paradigmatic, with associated hypothesis $\varphi$. For $\alpha<\omega_{1}$, we define the mouse operator $\mathcal{M}^{\alpha}$ by setting $\mathcal{M}^{0}=\mathcal{M}$, and for $x$ such that $\alpha$ is countable in the least admissible set over $x$ :

$$
\begin{aligned}
\mathcal{M}^{\alpha+1}(x)= & \text { minimal active } x \text {-mouse } \mathcal{P} \text { such that } \\
& \exists \gamma<\operatorname{crit}\left(\dot{F}^{\mathcal{P}}\right)\left(\mathcal{P} \text { is } \mathcal{M}^{\alpha} \text { - closed below } \gamma\right. \\
& \text { and } \mathcal{P} \models \varphi, \text { as witnessed by total extenders of } \mathcal{P} \mid \gamma) .
\end{aligned}
$$

For $\eta$ a limit ordinal which is countable in the least admissible set over $x$ :

$$
\mathcal{M}^{\eta}(x)=\bigcup_{\alpha<\eta} \mathcal{M}^{\alpha}(x)
$$

Theorem 6.5 Let $(\mathcal{M}, \lambda)$ be paradigmatic, and $D=D\left(\mathcal{M}_{\infty}^{I}, \lambda_{\infty}^{I}\right)$ where $I$ is $\mathcal{I}(\mathcal{M}, \lambda)$ generic. Then 
(a) if $\mathcal{M}=M_{a d r}^{\sharp}$, then for all $n<\omega$, the $\mathcal{M}^{n}$-operator is in $D$ and has Wadge rank $\theta_{n}$ in $D$;

(b) if $\mathcal{M}=M_{d c}^{\sharp}, M_{\text {inlim }}^{\sharp}$, or $M_{\text {wlim }}^{\sharp}$, then for all $\alpha<\omega_{1}$, the $\mathcal{M}^{\alpha}$ operator is in $D$, and has Wadge rank $\theta_{\alpha}$ in $D$.

Proof sketch. We show that (a) and (b) hold when $\alpha=n=1$, and leave the rest to the reader.

First, 3.6 generalizes in the following way.

Lemma 6.6 Let $I$ and $J$ be generic $\mathbb{R}$-genericity iterations of $\mathcal{M}(x)$ below $\lambda(x)$ and $\mathcal{M}^{1}(y)$ below $\lambda^{1}(y)$, respectively. Then $D\left(\mathcal{M}(x)_{\infty}^{I}, \lambda(x)_{\infty}^{I}\right)=D\left(\mathcal{M}^{1}(y)_{\infty}^{J}, \lambda^{1}(y)_{\infty}^{J}\right)$.

The proof is like that of 3.6. The main additional point is that an appropriate generic extension of $\mathcal{M}(x)$ can reconstruct $\mathcal{M}^{1}(y)$ arbitrarily high below $\lambda(x)$. This follows from 6.1 .

Next, 5.1 generalizes as follows:

Lemma 6.7 Under the hypotheses of theorem 6.5, we have that for $x, y$ countable transitive with $x \subseteq y$,

$$
x \in O D(z \mapsto \mathcal{M}(z), y)^{D} \Leftrightarrow x \in \mathcal{M}^{1}(y) .
$$

The proof is just like that of 5.1, using 6.6 in place of 3.6. These two lemmas imply that if the $\mathcal{M}^{1}$ operator is in $D$, then it has Wadge rank $\theta_{1}$ there. The proof is the same as that which shows the $\mathcal{M}^{0}$ operator sits at $\theta_{0}$.

To see that the $\mathcal{M}^{1}$ operator is in $D$, we relativise the argument of 6.1 . Let $\delta$ be the least Woodin cardinal above the least $<\lambda$-strong cardinal of $\mathcal{M}$, and let

$$
i: \mathcal{M} \rightarrow \mathcal{N}
$$

come from a genericity iteration below $\delta$ and above that strong cardinal, with $\mathcal{M} \in \mathcal{N}[g]$, where $g$ is $\operatorname{Col}(\omega, i(\delta))$-generic. We will show in the next section that

$$
\mathcal{N}[g]=\mathcal{M}^{1}[\langle\mathcal{N} \mid i(\delta), g\rangle]
$$

We can therefore apply the argument of 6.1 , doing a further genericity iteration $j: \mathcal{N} \rightarrow \mathcal{P}$ in the window between the first $<i(\lambda)$-strong cardinal of $\mathcal{N}[g]$ and its next Woodin, and add a universally Baire code of the $\mathcal{M}^{1}$ operator to some $\mathcal{P}[g][h]$. By derived model invariance, we may assume the $I$ in our theorem started out going to $\mathcal{N}$ and then $\mathcal{P}$, so we are done.

One can avoid using the identity $\mathcal{N}[g]=\mathcal{M}^{1}[\langle\mathcal{N} \mid i(\delta), g\rangle]$, borrowed from the next section, in the proof above. It is enough that $\mathcal{N}[g]$ can reconstruct an iterate $\mathcal{P}$ of $\mathcal{M}^{1}[\langle\mathcal{N} \mid i(\delta), g\rangle]$ via a construction which provides full background extenders for the total extenders on the $\mathcal{P}$-sequence. Note here that because of the background condition, every set in $V_{i(\lambda)} \cap \mathcal{N}[g]$ is generic over $\mathcal{P}$ by a forcing of size $<i(\lambda)$. This means that $\mathcal{P}$ is close enough to $\mathcal{N}[g]$ that the argument of 6.1 still works. 


\section{$7 \quad$ The $*$-transform}

In this section, we describe a method for translating mice with extenders overlapping some $\delta$ into relativised mice having $\delta$ as a cutpoint.

Suppose that $\mathcal{M}$ is an $\omega_{1}+1$-iterable, sound $x$-mouse, and $\mathcal{M}$ projects to $x$. Let $\mathcal{T}$ be a normal iteration tree on $\mathcal{M}$ played according to its unique iteration strategy, and let $\mathcal{N}$ be the last model of $\mathcal{T}$. Suppose $\delta$ is a cardinal of $\mathcal{N}$, and not measurable in $\mathcal{N}$ or any $\operatorname{Ult}(\mathcal{N}, E)$ with $\ln (E) \geq \delta$. Let $\tau$ be the order type of

$$
\begin{aligned}
S_{\delta}^{\mathcal{N}}= & \{\kappa<\delta \mid \exists E(E \text { is on the } \mathcal{N} \text {-sequence }) \\
& \text { and } \operatorname{crit}(E)=\kappa \text { and } \operatorname{lh}(E) \geq \delta\} .
\end{aligned}
$$

For $\alpha<\tau$, let

$$
\kappa_{\alpha}=\alpha^{\text {th }} \text { member of } S_{\delta}^{\mathcal{N}},
$$

and

$$
\gamma_{\alpha}=\text { least } \gamma \text { such that } \kappa_{\alpha}<\nu\left(E_{\gamma}^{\mathcal{T}}\right) \text {, }
$$

where we assume that $\gamma_{\alpha}$ exists for all $\alpha<\tau$, and

$$
\mathcal{P}_{\alpha}=\mathcal{M}_{\gamma_{\alpha}} \mid \xi_{\alpha}
$$

where $\xi_{\alpha}$ is the largest $\xi$ such that $\mathcal{M}_{\gamma_{\alpha}} \mid \xi$ and $\mathcal{M}_{\gamma_{\alpha}} \mid \operatorname{lh}\left(E_{\gamma_{\alpha}}^{\mathcal{T}}\right)$ have the same subsets of $\kappa_{\alpha}$. Note that $\mathcal{P}_{\alpha}$ is the model to which an extender with critical point $\kappa_{\alpha}$ would be applied in any normal continuation of $\mathcal{T}$. We now define

$$
\Phi_{\delta}(\mathcal{T})=\left\langle\mathcal{P}_{\alpha} \mid \alpha<\tau\right\rangle
$$

$\Phi_{\delta}(\mathcal{T})$ is essentially the sub-phalanx of $\Phi(\mathcal{T})$ containing the models we might actually go back to in a normal continuation of $\mathcal{T}$ using extenders of length $\geq \delta$. For example, if $\mathcal{T}$ is the genericity iteration of $\mathcal{M}$ we used in the proof of 6.1 , which lived in the window between the least $<\lambda$-strong of $\mathcal{M}$ and the next Woodin $\delta$, and $i: \mathcal{M} \rightarrow \mathcal{N}$ is the iteration map of $\mathcal{T}$, then $\Phi_{i(\delta)}(\mathcal{T})=\langle\mathcal{M}\rangle$.

Theorem 7.1 (Closson, Neeman, Steel) If $\mathcal{M}, \mathcal{T}, \mathcal{N}$, and $\delta$ are as above, and $g$ is $\operatorname{Col}(\omega, \xi)$ generic over $\mathcal{N}$, for some $\xi \leq \delta$, and $\Phi_{\delta}(\mathcal{T})$ is in the least admissible set over $\langle\mathcal{N} \mid \delta, g\rangle$, then

$$
\mathcal{N}[g]{ }^{*} \mathcal{R}
$$

for some $\langle\mathcal{N} \mid \delta, g\rangle$-mouse $\mathcal{R}$. 
Remark 7.2 The equality $\mathcal{N}[g]=\mathcal{R}$ cannot literally be true, since the two are structures for different languages. What we mean is that the two structures are fine-structurally equivalent, in that they have the same projecta, standard parameters, and Levy hierarchy past some point. (It can happen that their universes are different, although they are the same if $\mathcal{N}[g]$ is admissible.) We prefer not to spell out here the details of this notion of equivalence, which we call intertranslatability. We write $={ }^{*}$ for it.

Proof. We define the $*$-transform, which associates to initial segments $\mathcal{Q}[g]$ of $\mathcal{N}[g]$ mice $\mathcal{Q}[g]^{*}$ over $\langle\mathcal{N} \mid \delta, g\rangle$, in such a way that $\mathcal{Q}[g]$ and $\mathcal{Q}[g]^{*}$ are intertranslatable. Let $\Phi_{\delta}(\mathcal{T})=$ $\left\langle\mathcal{P}_{\alpha} \mid \alpha<\tau\right\rangle$.

To begin with, letting $\alpha>\delta$ be least such that $\mathcal{N} \mid \alpha \models \mathrm{KP}$, there is clearly a unique mouse $\mathcal{R}$ over $\langle\mathcal{N} \mid \delta, g\rangle$ such that $\mathcal{N} \mid \alpha[g]={ }^{*} \mathcal{R}$, and we let $(\mathcal{N} \mid \alpha[g])^{*}$ be this unique $\mathcal{R}$.

Let $U$ be the tree of all finite sequences $\left\langle E_{0}, \ldots, E_{n}\right\rangle$ such that each $E_{i}$ is an extender with $\operatorname{crit}\left(E_{i}\right)<\delta$ and $\operatorname{lh}\left(E_{i}\right) \geq \delta$, and $E_{0}$ is on the $\mathcal{N}$-sequence, and $E_{i+1}$ is on the sequence of $\operatorname{Ult}\left(\mathcal{M}_{\gamma_{\alpha}}, E_{i}\right)$ for $\alpha$ such that $\operatorname{crit}\left(E_{i}\right)=\kappa_{\alpha}$. For $\left\langle E_{0}, \ldots, E_{n}\right\rangle$ in $U$, we write $\mathcal{P}(\vec{E})$ for $\operatorname{Ult}\left(\mathcal{M}_{\gamma_{\alpha}}, E_{n}\right)$, where $\kappa_{\alpha}=\operatorname{crit}\left(E_{n}\right)$. Here we understand that $\mathcal{P}(\emptyset)=\mathcal{N}$. Because our initial $\mathcal{M}$ is iterable, $U$ is wellfounded. We shall define $\mathcal{Q}[g]^{*}$ for all $\mathcal{Q} \unlhd \mathcal{P}(\vec{E})$ where $\vec{E} \in U$ such that $o(\mathcal{Q})$ is at least the first admissible over $\mathcal{N} \mid \delta$. The definition is by induction on the $U$-rank of $\vec{E}$, with a subinduction on $o(\mathcal{Q})$.

The inductive clauses are as follows:

(a) if $o(\mathcal{Q})=\omega \alpha+\omega$, then $\mathcal{Q}[g]^{*}$ is obtained from $\mathcal{Q} \mid \alpha[g]^{*}$ by taking one step in the $J$-hierarchy.

(b) if $o(\mathcal{Q})$ is a limit ordinal and $\mathcal{Q}$ is passive, then $\mathcal{Q}[g]^{*}=\bigcup\left\{(\mathcal{Q} \mid \eta)[g]^{*} \mid \eta<o(\mathcal{Q})\right\}$.

(c) if $\mathcal{Q}$ is active with last extender $E$, and $\operatorname{crit}(E)>\delta$, then letting $\mathcal{Q}=(\mathcal{R}, E)$, we set $\mathcal{Q}[g]^{*}=\left(\mathcal{R}[g]^{*}, E\right)$.

(d) if $\mathcal{Q}$ is active with last extender $E$, and $\operatorname{crit}(E)=\kappa_{\alpha}<\delta$, then $\mathcal{Q}[g]^{*}=\operatorname{Ult}_{n}\left(\mathcal{P}_{\alpha}, E\right)[g]^{*}$, where $n$ is least such that $\rho_{n+1}\left(\mathcal{P}_{\alpha}\right) \leq \kappa_{\alpha}$.

Our non-measurability assumption on $\delta$ guarantees that these cases are exhaustive.

The detailed verification that $\mathcal{Q}[g]$ and $\mathcal{Q}[g]^{*}$ are intertranslatable takes some work. However, the basic idea is quite simple. Since $\Phi_{\delta}(\mathcal{T})$ is coded into $\langle\mathcal{Q} \mid \delta, g\rangle, \mathcal{Q}[g]$ can recover $\mathcal{Q}[g]^{*}$ by employing the inductive definition we just gave. Conversely, if we want to recover $\mathcal{Q}[g]$ from $\mathcal{Q}[g]^{*}$, all is trivial unless inductive clause (d) applies. Adopting the notation there, we simply note that $\mathcal{Q}^{*}$ can recover $\mathcal{P}_{\alpha}$ and $E$ as an appropriate core of itself, and associated core-embedding extender. It is worth noting that in this direction, we do not need to use $g$. 
(For example, let $\mathcal{Q}$ be the first level of $\mathcal{N}$ such that case (d) applies in defining $\mathcal{Q}[g]^{*}$. It is not hard to see that $\operatorname{crit}(E)=\kappa_{0}$ then. Let $n$ be least such that $\rho_{n+1} \leq \kappa_{0}$. We have $\operatorname{Ult}_{n}\left(\mathcal{P}_{0}, E\right)[g]^{*}=\operatorname{Ult}_{n}\left(\mathcal{P}_{0}, E\right)[g]$ because $E$ is the first extender overlapping $\delta$. We can regard $\operatorname{Ult}_{n}\left(\mathcal{P}_{0}, E\right)[g]$ as a mouse $\mathcal{Q}[g]^{*}$ over $\langle\mathcal{N} \mid \delta, g\rangle$. The universes of $\mathcal{Q}[g]$ and $\mathcal{Q}[g]^{*}$ are different in this case, but one has that the $r \Sigma_{n+1}^{\mathcal{Q}[g]^{*}}$ subsets of $\delta$ are the same as the $r \Sigma_{1}^{\mathcal{Q}[g]}$ subsets of $\delta$. Moreover, $\rho_{n+1}\left(\mathcal{Q}[g]^{*}\right) \leq \delta$, and $\mathcal{Q}[g]^{*}$ is $n+1$-sound as a mouse over $\langle\mathcal{N} \mid \delta, g\rangle$. Similarly, $\rho_{1}(\mathcal{Q}) \leq \delta$, and $\mathcal{Q}$ is 1 -sound. So $\mathcal{Q}[g]$ and $\mathcal{Q}[g]^{*}$ are intertranslatable.)

We can use the $*$-transform to compute the length of certain Solovay sequences.

Theorem 7.3 (a) For $\mathcal{M}=M_{a d r}^{\sharp}$ and $\lambda$ the sup of its Woodins, $D(\mathcal{M}, \lambda) \models \theta=\theta_{\omega}$.

(b) For $\mathcal{M}=M_{d c}^{\sharp}$ or $\mathcal{M}=M_{\text {inlim }}^{\sharp}$, and $\lambda$ the sup of its Woodins in either case, we have $D(\mathcal{M}, \lambda) \models \theta=\theta_{\omega_{1}}$.

Proof. We prove (b), the proof of (a) being similar. Let $I$ be $\mathcal{I}(\mathcal{M}, \lambda)$-generic, and $A \in \operatorname{Hom}_{I}^{*}$. By 6.5, it suffices to show that $A$ is projective in some operator $\mathcal{M}^{\tau}$, where $\tau<\omega_{1}$.

Let $A$ have a $<\lambda_{\infty}$-UB code in $M_{\infty}[g]$, where $g$ is generic on $\operatorname{Col}\left(\omega, \mu_{0}\right)$. Let $n$ be large enough that $\mu=\operatorname{crit}\left(i_{n, \infty}\right)>\mu_{0}$. Let $\mathcal{T}$ be the iteration tree giving rise to $i_{0, n}$. Let $\xi$ be the least Woodin of $M_{n}^{I}$ above $\mu$. We can do a genericity iteration of $M_{n}^{I}$ in the window $(\mu, \xi)$, producing a normal tree $\mathcal{U}$ and associated embedding $k: M_{n}^{I} \rightarrow \mathcal{P}$ so that $\Phi_{\mu}(\mathcal{T}) \in \mathcal{P}[g][h]$ for some $h$ which is $\mathcal{P}$ - generic over $\operatorname{Col}(\omega, k(\xi))$.

Let $\gamma$ be the least Woodin of $\mathcal{P}$ strictly above $k(\xi)$, and let $\Sigma$ be the iteration strategy for $\mathcal{M}$. By the proof of 3.0.1, $A$ is projective in the window-based fragment $\Gamma=$ $\Sigma \mid(\mathcal{T} \mathcal{U},[k(\xi), \gamma])$, and so it suffices to show this fragment is projective in some $\mathcal{M}^{\tau}$. Let $\tau$ be the order type of the cardinals of $\mathcal{P}$ which are strong past $\gamma$. Note that because $\xi$ and $\gamma$ were chosen to be least, the cardinals of $\mathcal{P}$ which are strong past $\gamma$ are all $<\mu$, and moreover

$$
\Phi_{\gamma}(\mathcal{T} \smile \mathcal{U})=\Phi_{\mu}(\mathcal{T})
$$

We can compute $\Gamma$ from $\mathcal{M}^{\tau}$ using $\mathcal{Q}$-structures. Letting $\mathcal{W}$ be played according to $\Gamma$, and $b=\Gamma(\mathcal{W})$, we have that $b$ is the unique cofinal branch $c$ of $\mathcal{W}$ such that $\mathcal{Q}(c, \mathcal{W})$ is iterable when backed up by the phalanx $\Phi_{\delta(\mathcal{W})}(\mathcal{T} \mathcal{U} \mathcal{\mathcal { W }})=\Phi_{\mu}(\mathcal{T})$. (We use here that $\mathcal{W}$ does not drop.) The branch oracle $\mathcal{Q}(b, \mathcal{W})$ may involve extenders overlapping $\delta(\mathcal{W})$, but these can be transformed away, and we get $b$ is the unique cofinal branch $c$ of $\mathcal{W}$ such that $\mathcal{Q}(\mathcal{W})[g][h]^{*} \unlhd \mathcal{M}^{\tau}(\langle\mathcal{M}(\mathcal{W}),(g, h)\rangle)$. The key here is that $\mathcal{Q}(\mathcal{W})[g][h]^{*}$ reaches no further than this in the hierarchy of mice over $\langle\mathcal{M}(\mathcal{W}),(g, h)\rangle)$. That can be proved by looking a little more closely at the definition of the $*$-transform.

Remark 7.4 The author first discovered a verion of the way $\mathcal{Q}[g]^{*}$ recovers $\mathcal{Q}[g]$ which works without the $g$ coding $\Phi_{\delta}(\mathcal{T})$, and used this to prove 7.3. Itay Neeman then observed that the 
process simplified considerably in the presence of a $g$ coding $\Phi_{\delta}(\mathcal{T})$, and would likely lead in that case to a level-by-level intertranslation. Erik Closson worked out the intertranslation in full detail.

\section{A long Solovay sequence}

We shall show in this section that $\theta_{\omega_{1}}<\theta$ in the derived model associated to $M_{\mathrm{w}}^{\sharp}$,im , and then give some indication as to how to prove that in fact $\theta=\theta_{\theta}$ holds there.

It is easy to say what the mouse operator sitting at $\theta_{\omega_{1}}$ is. Let us fix $\mathcal{M}=M_{\text {wlim }}^{\sharp}$ throughout this section, and let the $\mathcal{M}^{\alpha}$-operator be obtained from $\mathcal{M}$ as in 6.4.

Definition 8.1 For any countable transitive set $x$,

$$
\mathcal{M}^{\omega_{1}}(x)=\mathcal{M}^{\omega_{1}^{x}}(x),
$$

where $\omega_{1}^{x}$ is the height of the least admissible set to which $x$ belongs.

Clearly $\mathcal{M}^{\alpha}$ is projective in $\mathcal{M}^{\omega_{1}}$, for all $\alpha<\omega_{1}$. It is enough then to show that, letting $\lambda$ be the Woodin limit of Woodins in $\mathcal{M}$, and $D=D\left(\mathcal{M}_{\infty}^{I}, \lambda_{\infty}^{I}\right)$ where $I \in \mathcal{I}(\mathcal{M}, \lambda)$-generic, that $\mathcal{M}^{\omega_{1}} \in D$.

Claim 1. If $\mathcal{M}^{\omega_{1}} \uparrow V_{\lambda_{\infty}}^{M_{\infty}[g]} \in M_{\infty}[g]$, for some $g$ generic over $M_{\infty}$ for a poset of size $<\lambda_{\infty}$, then $\mathcal{M}^{\omega_{1}} \in D$.

Proof. The same proof that worked for $\mathcal{M}^{0}$ in 6.1 works here. Since $\mathcal{M}^{\omega_{1}}$ condenses to itself, and determines itself on small generic extensions, we get club many generically correct hulls of $M_{\infty}[g]$, and hence a UB code in $M_{\infty}[g]$ for $\mathcal{M}^{\omega_{1}}$.

Now let

$$
\mathcal{M} \models \kappa \text { is } \lambda+\omega \text {-reflecting in } \lambda \text {. }
$$

That there is such a $\kappa$ follows from the Woodinness of $\lambda$, and this is all of the Woodin property we shall need to show that $\mathcal{M}^{\omega_{1}} \in D$. Let $\xi$ be the least Woodin of $\mathcal{M}$ above $\kappa$, and let

$$
k: \mathcal{M} \rightarrow \mathcal{P}
$$

come from a genericity iteration $\mathcal{T}$ on $\mathcal{M} \mid \xi$ with all critical points $>\kappa$, such that

$$
\mathcal{M} \in \mathcal{P}[g] \text {, where } g \text { is } \operatorname{Col}(\omega, k(\xi)) \text {-generic over } \mathcal{P} \text {. }
$$

We may as well assume that $\mathcal{T}$ is the first tree used in $I$, and therefore it is enough to show Claim 2. $\mathcal{M}^{\omega_{1}} \mid V_{k(\lambda)}^{\mathcal{P}[g]} \in \mathcal{P}[g]$. 
Proof. We show that $\mathcal{P}[g]$ can compute $\mathcal{M}^{\omega_{1}}(x)$ by using the $*$-transform. To this end, let $\eta$ be a successor cardinal of $\mathcal{P}[g]$ such that $k(\xi)<\eta<k(\lambda)$. Let $F_{\eta}$ be the first extender $F$ on the $\mathcal{P}$-sequence such that $\kappa<\operatorname{crit}(F) \leq \eta$ and $\operatorname{lh}(F) \geq \eta$, if there is one, and let $F_{\eta}$ be a principal ultrafilter otherwise. We have that $\operatorname{crit}\left(F_{\eta}\right)<\eta$ and $F_{\eta}$ is total on $\mathcal{P}$. Note that $\operatorname{crit}\left(F_{\eta}\right)>k(\xi)$. Set

$$
\mathcal{P}_{\eta}=\operatorname{Ult}\left(\mathcal{P}, F_{\eta}\right)
$$

The choice of $F_{\eta}$ guarantees that there are no extenders $G$ on the sequence of $\mathcal{P}_{\eta}$ such that $\kappa<\operatorname{crit}(G) \leq \eta$ and $\operatorname{lh}(G) \geq \eta$. Thus

$$
\Phi_{\eta}\left(\mathcal{T} \frown\left\langle F_{\eta}\right\rangle\right)=\langle\mathcal{M}\rangle
$$

and since $\mathcal{M}$ is coded into $\langle\mathcal{P} \mid k(\xi), g\rangle$, we can define the $*$-transform of $\mathcal{P}_{\eta}[g]$ at $\eta$ as in the proof of 7.1 . We write

$$
\mathcal{Q}[g]^{*, \eta}
$$

for the $\langle\mathcal{P} \mid \eta, g\rangle$-mouse we get by applying the transform at $\eta$ to an appropriate $\mathcal{Q}[g]$.

We now show by induction on $\alpha<k(\lambda)$ :

Subclaim. If $\eta$ is a successor cardinal of $\mathcal{P}$ such that $k(\xi)<\eta<k(\lambda)$, and $\alpha \leq \omega_{1}^{\langle\mathcal{P} \mid \eta, g\rangle}$, then $\mathcal{M}^{\alpha}(\langle\mathcal{P} \mid \eta, g\rangle) \unlhd \mathcal{Q}[g]^{*, \eta}$, for some proper initial segment $\mathcal{Q}$ of $\mathcal{P}_{\eta}$.

Proof. We have done the case $\alpha=0$ in the proof of 6.1. Let $\alpha$ be a limit ordinal, and $\alpha \leq \omega_{1}^{\langle\mathcal{P} \mid \eta, g\rangle}$. By induction, we get that the function $f(\beta)=\mathcal{M}^{\beta}(\langle\mathcal{P} \mid \eta, g\rangle)$, defined on all $\beta<\alpha$, is in $\mathcal{P}_{\eta}[g]$. But then $f \in \mathcal{P}_{\eta}[g]^{*, \eta}$, so $f \in \mathcal{Q}[g]^{*, \eta}$ for some $\mathcal{Q} \triangleleft \mathcal{P}_{\eta}$. Clearly then $\mathcal{M}^{\alpha}(\langle\mathcal{P} \mid \eta, g\rangle) \unlhd \mathcal{Q}[g]^{*, \eta}$.

Now suppose the subclaim holds for $\alpha$. Fix $\eta$ such that $k(\xi)<\eta<\lambda, \eta$ is a successor cardinal of $\mathcal{P}[g]$, and $\alpha<\omega_{1}^{\langle\mathcal{P} \mid \eta, g\rangle}$. We want to show $\mathcal{M}^{\alpha+1}(\langle\mathcal{P} \mid \eta, g\rangle)$ is a proper initial segment of $\mathcal{P}_{\eta}[g]^{*, \eta}$. Note that $\kappa$ is $k(\lambda)+\omega$ reflecting in $k(\lambda)$ in $\mathcal{P}_{\eta}$, as it is not moved by our embedding from $\mathcal{M}$ to $\mathcal{P}_{\eta}$. Let $A$ be the theory in $\mathcal{P}_{\eta} \mid(k(\lambda)+\omega)$ of parameters in $\mathcal{P}_{\eta} \mid k(\lambda)$, and let $E$ be an extender on the $\mathcal{P}_{\eta}$-sequence so that $\operatorname{crit}(E)=\kappa$, and

$$
i_{E}(A) \cap \eta^{+}=A \cap \eta^{+}
$$

holds in $\mathcal{P}_{\eta}$. Let

$$
\mathcal{Q}=\mathcal{P}_{\eta} \mid \ln (E) \text {. }
$$

It is enough to show $\mathcal{M}^{\alpha+1}(\langle\mathcal{P} \mid \eta, g\rangle) \unlhd \mathcal{Q}[g]^{*, \eta}$. But $\mathcal{Q}[g]^{*, \eta}=\operatorname{Ult}(M, E)[g]^{*, \eta}$, which reaches the Woodin-limit-of-Woodins hypothesis, so it is enough to show that $\operatorname{Ult}(M, E)[g]^{*, \eta}$ is $\mathcal{M}^{\alpha}$-closed.

Now by our induction hypothesis, $\mathcal{P}[g]=$ " for all $\beta \leq \alpha$, for all successor cardinals $\nu$ such that $k(\xi)<\nu<k(\lambda)$ and $\beta \leq \omega_{1}^{\langle\mathcal{P} \mid \nu, g\rangle}, \mathcal{P}_{\nu}[g]^{*, \nu}$ has a proper initial segment satisfying "I am $\mathcal{M}^{\beta}(\langle\mathcal{P} \mid \nu, g\rangle)$ ")". Let us call the sentence in quotes $\psi(k(\xi), \mathcal{M}, g, k(\lambda), \alpha)$, 
where we have displayed the parameters about which it speaks. We then get $\mathcal{P}_{\eta}[g] \models$ $\psi\left[k(\xi), \mathcal{M}, g, k(\lambda), i_{F_{\eta}}(\alpha)\right]$, using that $k(\xi), k(\lambda), \mathcal{M}$, and $g$ are fixed by $i_{F_{\eta}}$. But $i_{F_{\eta}}(\alpha) \geq \alpha$, so inspecting $\psi$, we see $\mathcal{P}_{\eta}[g] \models \psi[k(\xi), \mathcal{M}, g, k(\lambda), \alpha]$. Letting $\tau$ be a term such that $\mathcal{M}=\tau^{g}$, we can fix $p \in \operatorname{Col}(\omega, k(\xi))$ such that

$$
\mathcal{P}_{\eta} \models p \Vdash \psi(k(\xi), \tau, \dot{g}, k(\lambda), \alpha) .
$$

Because $i_{E}(A) \cap \eta^{+}=A \cap \eta^{+}$holds in $\mathcal{P}_{\eta}$,

$$
\operatorname{Ult}\left(\mathcal{P}_{\eta}, E\right) \models p \Vdash \psi(k(\xi), \tau, \dot{g}, k(\lambda), \alpha) .
$$

But $\mathcal{M}$ embeds into $\mathcal{P}_{\eta}$ with critical point $>\kappa$, and hence $\operatorname{Ult}(M, E)$ embeds into $\operatorname{Ult}\left(\mathcal{P}_{\eta}, E\right)$ with critical point $>\eta^{+, \mathcal{P}_{\eta}}$. Thus

$$
\operatorname{Ult}(\mathcal{M}, E) \models p \Vdash \psi(k(\xi), \tau, \dot{g}, k(\lambda), \alpha) .
$$

It is easy to see that this implies that $\operatorname{Ult}(\mathcal{M}, E)[g]$ is $\mathcal{M}^{\alpha}$-closed below $i_{E}(\lambda)$.

The subclaim completes the proof of Claim 2.

Now we may assume that the first iteration tree used in $I$ is the tree giving rise to $k$. In that case, we get the hypothesis of Claim 1 from Claim 2, and so we have $\mathcal{M}^{\omega_{1}} \in D$.

Now suppose $\gamma<\theta^{D}$. We want to show $\theta_{\gamma}<\theta$ holds in $D$. Assume first that there is a prewellorder $\leq^{*}$ of $\mathbb{R}$ of order type $\gamma$ such that $\leq^{*}$ has a UB code in $\mathcal{M}$, or more precisely, is captured by $(\emptyset, \emptyset)$ in the sense of the proof of 3.6. (This is true if $\leq^{*}$ is projective, for example.) For $x \in R$ and $n<\omega$, we define a mouse operator $\mathcal{M}^{x, n}$ which, we shall show, has Wadge rank at least $\theta_{\omega|x|+n}$ in $D$. (Here and below, $|x|$ is the rank of $x$ in $\leq^{*}$.)

We shall define mouse jump operators $\mathcal{N}^{x, n}$, and then let $\mathcal{M}^{x, n}$ be the HC-extension of $\left(\mathcal{N}^{x, n}\right)$. The operator $\mathcal{N}^{x, n}$ will be defined on the Turing cone above $x$. The definition of $\mathcal{N}^{x, n}$ proceeds by induction on the lexicographic order on $\mathbb{R} \times \omega$ determined by $\leq^{*}$. If $|x|=0$, then we set $\mathcal{N}^{x, 0}=\mathcal{M} \uparrow \mathbb{R}$. If $|x|>0$, then for $z \in \mathbb{R}$,

$$
\mathcal{N}^{x, 0}(z)=\bigcup\left\{\mathcal{N}^{y, n}(z) \mid y \leq_{T} x \wedge y<^{*} x \wedge n<\omega\right\}
$$

It is important here that $\mathcal{N}^{x, 0}$ is a mouse-jump operator itself, and for that one needs to use the definability of $\leq^{*} \uparrow\left\{y \mid y \leq_{T} z\right\}$ over $\mathcal{M}(z)$. This is where we use the assumption that $\leq *$ has a UB code in $\mathcal{M}$.

Finally,

$$
\begin{aligned}
\mathcal{N}^{x, n+1}(z)= & \text { minimal active }\left(\mathcal{N}^{x, n}\right)^{+} \text {-closed } z \text {-mouse } \mathcal{R} \\
& \text { such that } \mathcal{R} \models \text { there is a Woodin limit of Woodins. }
\end{aligned}
$$

One can show that the $\mathcal{M}^{x, n}$ are in $D$ by an argument like that given in the proof of claim 2 above. It is also not hard to show $\mathcal{M}^{x, n+1}$ is not ordinal definable from $\mathcal{M}^{x, n}$ and a 
real over $D$, and is essentially Wadge least with this property. (However, we have no reason to believe that $\mathcal{N}^{x, 0}$ is even approximately Wadge minimal among upper bounds for the set of all $\mathcal{N}^{y, n}$ such that $y<^{*} x$.

In order to remove the assumption that $\leq^{*}$ has a UB code in $\mathcal{M}$, we replace our Woodin limit mouse operator $\mathcal{M}$ with a hybrid mouse operator $\mathcal{M}^{\Sigma}$, where $\Sigma \in D$ is an iteration strategy with condensation such that $\leq^{*}$ is Wadge reducible to $\Sigma$. Here $\mathcal{M}^{\Sigma}(z)$ is the minimal active $\Sigma$-mouse with a Woodin limit of Woodins. The invariance of the derived model proof ( see 3.6) extends so as to show that $\mathcal{I}$-generic iterations of $\mathcal{M}^{\Sigma}(z)$ yield the derived model $D$ as well. This can be used as above to show that the nestings of $\mathcal{M}^{\Sigma}$ guided by $\leq^{*}$ are in $D$.

Remark 8.2 (a) Erik Closson has identified the least tractable $(\mathcal{M}, \lambda)$ such that $\theta_{\omega_{1}}<\theta$ holds in the derived model $D(\mathcal{M}, \lambda)$. The precise description is somewhat technical, but the existence of a $\kappa<\lambda$ which is $S$-strong in $\lambda$, where $S=\{\mu<\lambda \mid \mu$ is $<\lambda$-strong $\}$, is more than enough.

(b) The theory $\mathrm{AD}^{+}+\theta_{\omega_{1}}<\theta$ should be equiconsistent with the theory satisfied by the least mouse whose derived model satisfies $\theta_{\omega_{1}}<\theta$. This is open at the moment, however.

(c) We have no large-cardinal characterization of the least tractable $(\mathcal{M}, \lambda)$ such that $\theta_{\omega_{2}}<\theta$ holds in $D(\mathcal{M}, \lambda)$.

\section{The mouse set conjectures: framework of the induc- tion}

We now shift gears, and head toward some partial results on the mouse set conjectures MSC. We shall be assuming $\mathrm{AD}^{+}$for pretty much the rest of these notes, perhaps tacitly on occasion. We shall rely heavily on the concepts and results involved in Woodin's proof of the mouse set conjectures below iteration strategies for nontame mice, as presented in [23]. ( We give Woodin's proof at the end of section 11.)

For our arguments, it is important to prove a local form of 1.0.2. Let us say that $\Gamma$ is a boldface pointclass just in case $\Gamma$ is a collection of sets of reals closed downward under Wadge reducibility. By results of Wadge and Martin, the inclusion order on boldface pointclasses closed under complements is a prewellorder. A projectively closed pointclass is a boldface pointclass closed under complements and real quantification.

Definition 9.1 (AD) $P_{\alpha}$ is the $\alpha^{\text {th }}$ projectively closed pointclass, in the inclusion order. We associate a structure to $P_{\alpha}$ by setting

$$
\tilde{P}_{\alpha}=\left(H C \cup P_{\alpha}, \in\right) .
$$


So $P_{0}$ is the class of projective sets, and $P_{\lambda}=\bigcup_{\alpha<\lambda} P_{\alpha}$ for $\lambda$ limit.

Definition 9.2 Let $\varphi(v)$ be a $\Sigma_{n}$ formula in the language of set theory expanded by a unary predicate symbol $\dot{A}$, and let $y$ be countable and transitive. We call a pair $(\mathcal{M}, \Sigma) a\langle\varphi, y\rangle$ witness just in case

(a) $\mathcal{M}$ is an ms-mouse over $y$,

(b) $\Sigma$ is a good $\omega_{1}$-iteration strategy for $\mathcal{M}$, and

(c) for some $\lambda$,

$$
\mathcal{M}=\text { there are } n+5 \text { Woodin cardinals }<\lambda \text {, }
$$

and

$$
\mathcal{M} \models \exists A \in \operatorname{Hom}_{<\lambda}((H C, \in, A) \models \varphi[y]) .
$$

Although finer local versions of the mouse set conjectures could be stated and proved (in the initial segment of the Wadge hierarchy for which we have any proofs at the moment), we shall be content with the following.

Definition 9.3 $\mathrm{S}_{\lambda}$ is the assertion: for any real $y$, any $A \in P_{\lambda}$, and formula $\varphi(v)$, if $(H C, \in, A) \models \varphi[y]$, then there is a $\langle\varphi, y\rangle$-witness $(\mathcal{M}, \Sigma)$ such that $\Sigma \in P_{\lambda}$.

$\mathrm{S}_{\lambda}$ is a local version of MSC2. It easily implies a corresponding local version of MSC1.

Definition 9.4 We write $x \in O D^{\alpha}(y)$ just in case $x$ is definable from $y$ and ordinal parameters over $\tilde{P}_{\alpha}$. We write $x \in O D^{<\lambda}(y)$ just in case $x \in O D^{\alpha}(y)$ for some $\alpha<\lambda$.

Definition 9.5 $C_{\lambda}$ is the assertion: whenever $x$ and $y$ are countable transitive sets, with $x \subseteq y$, and $x \in O D^{<\lambda}(y)$, then there is an ms-mouse $\mathcal{M}$ over $y$ such that $x \in \mathcal{M}$, and $\mathcal{M}$ has an $\omega_{1}$-iteration strategy in $P_{\lambda}$.

Lemma 9.6 Let $\lambda$ be a limit ordinal, and suppose $\mathrm{S}_{\lambda}$ holds; then $\mathrm{C}_{\lambda}$ holds.

The lemma is an easy consequence of the fact that "there is a $\gamma$-th real in $\operatorname{OD}^{<\lambda}(y)$ " can be expressed by " $\tilde{P}_{\lambda}=\psi[y]$ ", for some $\Sigma_{1}$ formula $\psi$. We omit further proof.

In the remainder of this paper, we shall outline the proof of a special case of:

Theorem 9.7 (Neeman, Steel) Let $\lambda$ be a limit ordinal which is small enough that no $\omega_{1}$-iteration strategy for a mouse as in the hypothesis of 1.2 belongs to $P_{\lambda}$; then $\mathrm{S}_{\lambda}$ holds.

The proof goes by induction on $\lambda$. The important stages to consider are of course those at which new $\Sigma_{1}^{2}$ facts are verified. 
Definition 9.8 We write $\tilde{P}_{\alpha} \prec_{1}^{\mathbb{R}} \tilde{P}_{\beta}$ iff $\alpha \leq \beta$, and whenever $\phi(v)$ is $\Sigma_{1}, x \in \mathbb{R}$, and $\tilde{P}_{\beta} \models \phi[x]$, then $\tilde{P}_{\alpha} \models \phi[x]$.

Definition 9.9 Let $\alpha \leq \beta \leq \theta$; then we call $[\alpha, \beta]$ a $\Sigma_{1}^{2}$-gap iff

(1) $\tilde{P}_{\alpha} \prec_{1}^{\mathbb{R}} \tilde{P}_{\beta}$,

(2) there is no $\gamma<\alpha$ such that $\tilde{P}_{\gamma} \prec_{1}^{\mathbb{R}} \tilde{P}_{\alpha}$, and

(3) there is no $\gamma>\beta$ such that $\gamma \leq \theta$ and $\tilde{P}_{\beta} \prec_{1}^{\mathbb{R}} \tilde{P}_{\gamma}$.

Woodin's proofs that $\Sigma_{1}^{2}$ has the scale property and that every $\Sigma_{1}^{2}$ collection of sets of reals has a $\Delta_{1}^{2}$ member localize, giving

Theorem 9.10 (Woodin) Assume $\mathrm{AD}^{+}$; then for any $\alpha$

(a) the lightface pointclass $\Sigma_{1}^{\tilde{P}_{\alpha}}$ has the scale property, and

(b) if $x \in \mathbb{R}, \phi$ is $\Sigma_{1}$, and there is an $A \in P_{\alpha}$ such that $\tilde{P}_{\alpha} \models \phi[A, x]$, then there is a $\Delta_{1}^{\tilde{P}_{\alpha}}(x)$ set $A \in P_{\alpha}$ such that $\tilde{P}_{\alpha} \models \phi[A, x]$.

It follows that there is a last $\Sigma_{1}^{2}$ gap, namely $\left[\delta_{1}^{2}, \theta\right]$, where $\delta_{1}^{2}$ is the sup of the lengths of the boldface $\Delta_{1}^{2}$ prewellorders of the reals, or equivalently, the length of any regular $\Sigma_{1}^{2}$-norm on a complete $\Sigma_{1}^{2}$ set.

According to 9.10 , the appearance of a new $\Sigma_{1}^{2}$ truth about a real generates new scales. Indeed, suppose $\beta$ ends a $\Sigma_{1}^{2}$ gap, that is, it is not the case that $\tilde{P}_{\beta} \prec_{1}^{\mathbb{R}} \tilde{P}_{\beta+1}$. We get from 9.10(b) a $\Delta_{1}^{\tilde{P}_{\beta+1}}(x)$ set $A \subseteq \mathbb{R}$ such that $A \in P_{\beta+1} \backslash P_{\beta}$. It follows that every set in $P_{\beta+1}$ is in the scaled boldface pointclass $\Sigma_{1}^{\tilde{P}_{\beta+1}}(\mathbb{R})$. So the new $\Sigma_{1}^{2}$ truth witnessed by a set of reals in $P_{\beta+1}$ has quickly generated scales on all sets of reals in $P_{\beta+1}$.

In $L(\mathbb{R})$ the converse is true, in that no new scales appear in a $\Sigma_{1}^{2}$ gap. This is not true in general, however. If $\theta_{0}<\theta$, then there is a scale on $\Pi_{1}^{2}$ which appears inside the gap $\left[\delta_{1}^{2}, \theta\right]$; in fact the least Wadge rank of such a scale is $\theta_{0}$, and we get a pointclass with the scale property at that point. (These are consequences of $\mathrm{AD}^{+}$due to Woodin; see 9.18 below.) There can be local versions of this phenomenon in gaps below $\left[\delta_{1}^{2}, \theta\right]$. One way to understand the picture is to assume that our $\mathrm{AD}^{+}$universe is an initial segment of $K(\mathbb{R})$, as it probably must be unless we have reached $\omega_{1}$-iteration strategies for mice with superstrongs. ${ }^{2}$ As shown in [24], in initial segments of $K(\mathbb{R})$, new scales correspond precisely to the verification of new $\Sigma_{1}$ formulae about reals, in exactly the same pattern as held in $L(\mathbb{R})$. The rub is that these formulae are allowed a predicate for the extender sequence $\vec{E}$ from which $K(\mathbb{R})$ is constructed, and our initial segment of $K(\mathbb{R})$ may not have the iteration strategies needed

\footnotetext{
${ }^{2}$ In section 16 , we show that, essentially, this follows from MSC.
} 
to define $\vec{E}$. New scales appear in lock-step with the verification of new $\Sigma_{1}$ facts about $\vec{E}$, but these only generate new $\Sigma_{1}^{2}$ facts when $\omega_{1}$-iteration strategies for countable $M \prec K(\mathbb{R}) \mid \alpha$ not formerly seen to be $\omega_{1}$-iterable are constructed.

We begin now with our inductive proof of $S_{\lambda}$. If $\lambda$ is least limit ordinal such that $S_{\lambda}$ fails, then $\lambda=\gamma+\omega$ for some limit ordinal $\gamma$. Clearly, there is some least $\beta$ such that $\gamma \leq \beta<\lambda$ and it is not the case that $\tilde{P}_{\beta} \prec_{1}^{\mathbb{R}} \tilde{P}_{\beta+1}$; that is, there is a least $\beta \in[\gamma, \lambda)$ which ends a $\Sigma_{1}^{2}$ gap. Let $\beta^{*}$ be this $\beta$.

Let $\alpha^{*}$ begin the $\Sigma_{1}^{2}$ gap which ends at $\beta^{*}$. Let

$$
\bar{\Gamma}=\Sigma_{1}^{\tilde{P}_{\alpha^{*}}}
$$

be the scaled pointclass at the beginning of our gap. From this point through the end of section 14 , we have fixed $\alpha^{*}, \beta^{*}$, and $\bar{\Gamma}$, and we are trying to prove $\mathrm{S}_{\beta^{*}+\omega}$.

Lemma 9.11 If $\bar{\Gamma}$ is not closed under real quantification, then $\mathrm{S}_{\beta^{*}+\omega}$ holds.

Proof sketch. In this case we must have $\alpha^{*}=\beta^{*}$, and since we chose $\beta^{*}$ least, we must then have that $\beta^{*}$ is a limit ordinal. We therefore have $S_{\beta^{*}}$ by induction. We can now use arguments like those in the "no gap" case of the core model induction ( $[27])$ to get a mouse operator $\mathcal{M}$ such that $\mathcal{M}(z)$ knows $\Sigma_{1}^{\tilde{P}_{\beta^{*}+1}}(z)$ truth, and has an $\omega_{1}$ iteration strategy in $P_{\beta^{*}+1}$, for all $z$. Now we can use the argument of [23] to show that $\mathrm{S}_{\beta^{*}+\omega}$ fails, then there is an $\mathcal{M}$-closed mouse $\mathcal{N}$ with infinitely many Woodin cardinals and a measurable above such that $\mathcal{N}$ has an iteration strategy $\Sigma$ in $P_{\beta^{*}+\omega}$. This is impossible, as all sets in $L\left(P_{\beta^{*}+1}\right)$ are projective in any such $\Sigma$.

We therefore assume that $\bar{\Gamma}$ is closed under real quantification.

The rest of what we can prove has a vague similarity to the proof of 9.11 , in that we show that if the gap between $\bar{\Gamma}$, the pointclass we have already captured by mice, and $P_{\beta^{*}+\omega}$, the next pointclass to be captured, is not too large, then we can capture $P_{\beta^{*}+\omega}$ as desired (that is, $\mathrm{S}_{\beta^{*}+\omega}$ holds). The markers in our trek across this $\Sigma_{1}^{2}$ gap are the new scaled pointclasses. We now state some results of Martin and Woodin (see [8] and [3]) which characterize the next scaled pointclass after one closed under real quantifiers abstractly, in terms of reflection properties.

Definition 9.12 We call a pointclass good if it is $\omega$-parametrized, and closed under recursive substitution, number quantification, and existential real quantification. A good scaled pointclass is a good pointclass which has the Scale Property. An inductive-like pointclass is a good scaled pointclass closed under universal real quantification.

The scale property for $\bar{\Gamma}$ follows from 9.10, so we are assuming henceforth that $\bar{\Gamma}$ is inductive-like. 
Definition 9.13 Let $\Gamma$ be a good scaled pointclass, and $A \subseteq \mathbb{R}$. We say that $A$ is countably captured over $\Gamma$ just in case there is a real $x$ such that for all countable $\sigma \subseteq \mathbb{R}$ with $x \in \sigma$, $A \cap \sigma \in C_{\Gamma}(\sigma \cup\{\sigma\})$. Such a real $x$ we call a $\Gamma$-good parameter for $A$. We call $x$ a $\Gamma$-good parameter for the sequence $\vec{A}$ just in case it is a $\Gamma$-good parameter for each $A_{i}$.

Here $C_{\Gamma}(b)$ is the largest countable $\Gamma(b)$ collection of subsets of $b$, for $b$ countable transitive. $C_{\Gamma}(b)=P(b) \cap L[T, b]$ for $T$ the tree of a $\Gamma$-scale on a universal $\Gamma$ set. (Harrington and Kechris, [2].) It is clear that if $x$ is a $\Gamma$-good parameter for $A$, then so is any $y \geq_{T} x$. So if $x_{i}$ is $\Gamma$-good for $A_{i}$, then any real coding all the $x_{i}$ is $\Gamma$-good for $\vec{A}$.

Definition 9.14 For any good pointclass $\Gamma$, the envelope of $\Gamma$ is given by: $\operatorname{Env}(\Gamma)=\{A \mid$ $A$ is countably captured over $\Gamma\}$.

It is easy to see from the definition that $\operatorname{Env}(\Gamma)$ is a boldface pointclass closed under complements. Martin's argument from $[8, \S 4]$ shows

Theorem 9.15 (Martin [8]) If $\Gamma$ is closed under real quantifiers, then

(a) $\operatorname{Env}(\Gamma)$ is closed under real quantifiers, and hence a projectively closed boldface pointclass, and

(b) there is no scale on a universal $\Gamma$-dual set whose sequence of associated prewellorders is in $\operatorname{Env}(\Gamma)$.

We include a proof of (b), because it is short: if (b) fails, then the relation $x \notin C_{\Gamma}(y)$ would be uniformized by a function $f \in \operatorname{Env}(\Gamma)$. The relation $R(y, n, m) \Leftrightarrow f(y)(n)=m$ would then be countably captured over $\Gamma$, and letting $y_{0}$ be a real which witnesses this, we would have $f\left(y_{0}\right) \in C_{\Gamma}\left(y_{0}\right)$, a contradiction.

The next scaled pointclass after one closed under real quantifiers lies just beyond its envelope, and is given by a self-justifying system.

Definition 9.16 A self-justifying system is a countable set $\mathcal{A} \subseteq P(\mathbb{R})$ which is closed under complements (in $\mathbb{R}$ ), real quantification, and such that every $A \in \mathcal{A}$ admits a scale $\vec{\psi}$ such that $\leq_{\psi_{i}} \in \mathcal{A}$ for all $i$. We say a sequence $\vec{A}=\left\langle A_{i} \mid i<\omega\right\rangle$ is self-justifying iff $\left\{A_{i} \mid i<\omega\right\}$ is self-justifying. An sjs is a self-justifying system or sequence.

Definition 9.17 Let $\Gamma$ be a good scaled pointclass pointclass, and let $\mathcal{A}$ be an sjs. We say that $\mathcal{A}$ seals $\Gamma$ just in case there is a universal $\Gamma$ set in $\mathcal{A}$, and $\mathcal{A} \subseteq \operatorname{Env}(\Gamma)$. 
The following is the basic result on the existence of self justifying systems which seal a given pointclass. It was proved by Woodin in the mid 90's. ${ }^{3}$ The proof uses the method of obtaining Suslin representations from direct limits of mice. The last section of [26] constructs an sjs which seals some $\Gamma$ by very much the same method.

Theorem 9.18 (Woodin) Assume $\mathrm{AD}^{+}$. Let $\alpha$ begin a $\Sigma_{1}^{2}$-gap, and $\mathrm{S}_{\alpha}$ holds. Let $\Gamma=$ $\left(\Sigma_{1}\right)^{\tilde{P}_{\alpha}}$. Suppose $\Gamma$ is closed under real quantification, and suppose there are scaled sets which are not in $\Gamma$; then for any $B \in \Gamma$, there is a self-justifying system $\mathcal{A}$ such that $B \in \mathcal{A}$, and $\mathcal{A}$ seals $\Gamma$.

It is not hard to see that if $\mathcal{A}$ seals $\Gamma$, then letting $\Gamma^{+}$be the collection of sets of reals which are Wadge reducible to a countable union of sets in $\mathcal{A}$, we have that $\Gamma^{+}$is the least boldface pointclass with the scale property and closed under countable unions properly including $\Gamma$.

Our goal in these notes will be to show that $\mathrm{S}_{\beta^{*}+\omega}$ holds as long as there is no local $\theta_{0}$ between $\alpha^{*}$ and $\beta^{*}$ :

Assumption $(\dagger)$ : There is no $\xi$ such that $\alpha^{*}<\xi \leq \beta^{*}, P_{\xi}=P(\mathbb{R}) \cap L\left(P_{\xi}\right)$, and $L\left(P_{\xi}\right) \models$ $\mathrm{AD}^{+}+\theta_{0}<\theta$.

One can formulate this in terms of mice as: $\mathrm{S}_{\beta^{*}+\omega}$ holds so long as there is no iteration strategy in $P_{\beta^{*}+\omega}$ for a mouse $\mathcal{M}$ having a limit of Woodin cardinals $\lambda$, some cardinal $\kappa<\lambda$ which is $<\lambda$ strong, and such that $\mathcal{M}$ is closed under lower part mice built over its initial segments below $\lambda$ which have iteration strategies in $\bar{\Gamma}$. We shall not need the equivalence of $(\dagger)$ with this statement, however.

Recall that $\bar{\Gamma}=\left(\Sigma_{1}^{2}\right)^{P_{\alpha^{*}}}=\left(\Sigma_{1}^{2}\right)^{P_{\beta^{*}}}$. By 9.10 every $\bar{\Gamma}$-dual set admits a scale (in $P_{\beta^{*}+1}$ ), and thus by 9.18 , there is a sjs $\vec{A}$ such that $\vec{A}$ seals $\bar{\Gamma}$. Assumption (†) implies at once that

$$
P_{\beta^{*}+\omega} \subseteq L(\vec{A}, \mathbb{R}),
$$

for any such sjs $\vec{A}$. For otherwise, $P(\mathbb{R}) \cap L(\vec{A}, \mathbb{R}) \subseteq P_{\beta^{*}}$ by Wadge. But then $\bar{\Gamma}=\left(\Sigma_{1}^{2}\right)^{L(\vec{A}, \mathbb{R})}$, and hence in $L(\vec{A}, \mathbb{R}), \vec{A}$ yields a scale on a universal $\Pi_{1}^{2}$ set. Thus $L(\vec{A}, \mathbb{R}) \models \theta_{0}<\theta$, and letting $\xi$ be such that $P_{\xi}=P(\mathbb{R}) \cap L(\vec{A}, \mathbb{R})$, we have a counterexample to $(\dagger)$.

With regard to our trek upward along scales from $\bar{\Gamma}$ to $P_{\beta^{*}+\omega}$, assumption $(\dagger)$ says that we have one potentially significant leap, from $\bar{\Gamma}$ to some $\vec{A}$ which seals it, followed by steps within $L(\vec{A}, \mathbb{R})$. The latter steps are easily understood, in the same way that steps within $L(\mathbb{R})$ are understood.

We shall first prove what might seem like a weak approximation to $\mathrm{C}_{\beta^{*}+\omega}$ :

\footnotetext{
${ }^{3}$ Steve Jackson's article ([3, Lemma 3.18]) exposits some older results of Martin which approximate the result. In the notation of [3], $\operatorname{Env}(\Gamma)$ is $\Lambda(\Gamma, \kappa)$ for $\kappa$ the prewellordering ordinal of $\Gamma$. It is introduced by a somewhat different definition, ostensibly stronger than countable capturing. One can use 9.15 to show the two definitions equivalent. Jackson also found a direct proof of the equivalence.
} 
Lemma 9.19 If assumption $(\dagger)$ holds, then for a Turing cone of reals $x$, there is a ms-mouse $\mathcal{M}_{x}$ over $x$ such that

(a) $\mathcal{M}_{x}$ projects to $\omega$,

(b) $\mathcal{M}_{x}$ has an $\omega_{1}$-iteration strategy in $P_{\beta^{*}+\omega}$, but

(c) $\mathcal{M}_{x}$ has no $\omega_{1}$-iteration strategy in $P_{\alpha^{*}}$.

We shall show in section 14 that in fact the conclusion of Lemma 9.19 implies $\mathrm{S}_{\beta^{*}+\omega}$, in general, without the restrictive assumption $(\dagger)$. (But assuming $\mathrm{AD}^{+}$, of course.) Our proof of 9.19 will take up sections $10-13$. In these sections we assume $\mathrm{AD}^{+}$, and that $\alpha^{*}, \beta^{*}$, and $\bar{\Gamma}$ are as above.

\section{The background universe $N_{x}^{*}$}

As in [23], we will produce the ms-mice verifying 9.19 by using a full background extender construction, done in a sufficiently strong background universe.

Definition 10.1 Let $M$ be a countable transitive model of ZFC, let $\Sigma$ be an $\left(\omega, \omega_{1}\right)$-iteration strategy for $M$, and let $A \subseteq \mathbb{R}$. Then we say $(M, \Sigma)$ captures $A$ (at $\delta$, via $(T, U)$ ) just in case

(a) $M \models$ " $\delta$ is Woodin, and $(T, U)$ are absolutely complementing for $\operatorname{Col}(\omega, \delta)$ ", and

(b) for all reals $x, x \in A$ iff there is an iteration map $i: M \rightarrow P$, coming from an iteration according to $\Sigma$, such that $x \in p[i(T)]$.

If $A$ is captured by some $(M, \Sigma)$ such that $\Sigma$ has the Dodd-Jensen property, then $A$ is Suslin and co-Suslin. (The Dodd-Jensen property lets us define a direct limit for the system of all countable iterates of $M$ via $\Sigma$. Letting $\left(T_{\infty}, U_{\infty}\right)$ be the image of $(T, U)$ in this direct limit, we can use genericity iterations to see that $\left(T_{\infty}, U_{\infty}\right)$ witnesses that $A$ is Suslin and co-Suslin.) Conversely, it is a basic result of Woodin that assuming $\mathrm{AD}^{+}$, every Suslin and co-Suslin set $A$ is captured by some $(M, \Sigma)$ such that $\Sigma$ has the Dodd-Jensen property. In fact

Theorem 10.2 (Woodin, see [5] and [23].) Assume $\mathrm{AD}^{+}$, let $\Gamma$ be a good scaled pointclass not closed under $\forall^{\mathbb{R}}$, and let $A \in \Gamma$. Then for any real $x$, there is an $(M, \Sigma)$ such that

1. $x \in M$, and $(M, \Sigma)$ captures $A$, 
2. $\Sigma$ has condensation, and hence the Dodd-Jensen property, and

3. $\Sigma$ is projective in $\Gamma$.

See lemma 3.12 of [23] for a proof of 10.2. That proof relies heavily on [5]. MSC implies a fine-structural strengthening of 10.2 ; see theorem 16.6 below.

We shall be dealing with relativised, hybrid mice $M$ of various types below. They are relativised in that they may be built over some countable transitive $x$, which is usually selfwellordered, so that the resulting model satisfies AC. Our convention is that the language of a mouse built over $x$ has a name for $x$ (but not names for elements of $x$ ). Our mice may be hybrids, in that at successor steps we may be closing under something stronger than first order definability (while occasionally adding extenders at limit steps). The function we close under at successor steps must have condensation properties which yield a good theory of the resulting hybrid mice.

In this situation we shall write $M \mid \eta$ for the $\eta$-th level of $M$.

We shall also deal with hybrid mouse operators, that is, functions $x \mapsto M_{x}$, where $M_{x}$ is a hybrid mouse over $x$, defined on an HC-cone of countable transitive $x$.

We need a strengthening of 10.2, one in which the capturing mouse knows something about its own iteration strategy. This comes from combining 10.2 with Woodin's analysis of HOD. (See [5], [19, §8], [22].)

Theorem 10.3 (Woodin) Assume $\mathrm{AD}^{+}$, let $\Gamma$ be a good scaled pointclass not closed under $\forall^{\mathbb{R}}$, and let $A \in \Gamma$. Then there is a function $F(x)=\left(M_{x}, N_{x}^{*}, \Sigma_{x}, \delta_{x}\right)$, defined on a cone of countable transitive $x$, such that $F \in L_{2}(\Gamma, \mathbb{R})$ (when coded naturally as a set of reals), and

(a) $\left(M_{x}, \Sigma_{x}\right)$ captures $A$ at $\delta_{x}$ via some $(T, U)$, and

(b) $x \in M_{x}, \Sigma_{x} \uparrow V_{\delta_{x}}^{M_{x}} \in M_{x}$,

(c) $N_{x}^{*}=L\left(M_{x}, \Lambda\right)$, where $\Lambda$ is the restriction of $\Sigma_{x}$ to finite stacks $\overrightarrow{\mathcal{T}}$ of normal iteration trees based on $V_{\delta_{x}}^{M_{x}}$ such that $\overrightarrow{\mathcal{T}} \in M_{x}$,

(d) $V_{\delta_{x}}^{M_{x}}=V_{\delta_{x}}^{N_{x}^{*}}, M_{x}\left|\delta_{x}=N_{x}^{*}\right| \delta_{x}$, and

(e) $N_{x}^{*} \models \mathrm{ZFC}+\delta_{x}$ is Woodin.

Proof sketch. For those to whom it might make sense, here is a very rough sketch. Let $\left\langle\Gamma_{i}\right\rangle$ be a strictly increasing sequence of good scaled pointclasses, each containing $A$, and projective in $\Gamma$ (i.e., in $\left.\mathrm{i} L_{1}(\Gamma, \mathbb{R})\right)$. Let $J_{i}$ be an iteration strategy with condensation for coarse $\Gamma_{i}$ Woodin mouse $Q_{i}$. (See lemma 3.12 of [23] for the construction of such $J_{i}$ and $Q_{i}$.) Letting $J=\left\langle J_{i} \mid i<\omega\right\rangle$ and $Q=\left\langle Q_{i} \mid i<\omega\right\rangle$, we can, for any real $x$, build $J$-hybrid mice over 
$\langle Q, x\rangle$. These are mice over $\langle Q, x\rangle$ constructed from extender sequences as usual, except that the mice are told the action of the iteration strategies in $J$ on trees which they construct. The condensation properties of $J$ guarantee that if this is done properly, the resulting mice have a fine structure, and behave like ordinary ms-mice. The desired $M_{x}$ in 10.3 is then $\mathcal{M}_{1}^{J}(x) \mid \kappa_{x}$, the minimal iterable 1 - $J$-Woodin hybrid mouse over $\langle Q, x\rangle$, cut off at its least inaccessible cardinal $\kappa_{x}$ above its Woodin cardinal. Such an iterable hybrid $J$-Woodin mouse exists, and has an iteration strategy in $P_{\beta^{*}+\omega}$, because we can simply repeat the construction in lemma 3.12 of [23] in the hybrid setting. Let $\delta_{x}$ be the Woodin cardinal of $M_{x}$, and let $\Sigma_{x}$ be its canonical iteration strategy. Because $M_{x}$ knows $J$, and $\Sigma_{x}$ moves $J$ properly, we get that $\left(M_{x}, \Sigma_{x}, \delta_{x}\right)$ captures $A$.

Finally, we set $N_{x}^{*}=L\left(M_{x}, \Lambda\right)$, where $\Lambda$ is the restriction of $\Sigma_{x}$ to iteration trees $\mathcal{T}$ based on $V_{\delta_{x}}^{M_{x}}$ such that $\mathcal{T} \in M_{x}$. The key is that for a cone of $x$, adding $\Sigma_{x}$ to $M_{x}$ neither adds bounded subsets to $\delta_{x}$, nor kills the Woodiness of $\delta_{x}$. This is is a direct limit system argument involving ideas in the computation of $\operatorname{HOD}^{L[x, G]}$, which Woodin used to prove the corresponding theorem about adding an appropriate fragment of the canonical iteration strategy for $M_{1}(x)$ to $M_{1}(x) .{ }^{4}$

This end our sketch.

Remark 10.4 It should be possible to improve 10.3 by requiring that $F$ be projective in $\Gamma$, rather than one step away from that. It is because we don't have this stronger result that we need the room we get by only trying to prove $S_{\lambda}$ for limit $\lambda$. Presumably there is a way to use more care here, and get $S_{\eta}$ for all $\eta$. At any rate, the argument of section 15 shows $\mathrm{S}_{\beta^{*}+\omega}$ implies $S_{\beta^{*}+n}$ for all $n$.

Given $A \in P_{\beta^{*}+\omega}, 10.3$ gives us for a cone of $x$ a background universe $N_{x}^{*}$ having $x$ in it which

(i) has a Woodin cardinal $\delta_{x}$,

(ii) knows how to iterate itself for finite stacks $\overrightarrow{\mathcal{T}}$ of normal trees based on $N_{x}^{*} \mid \delta_{x}$ such that $\overrightarrow{\mathcal{T}} \in L_{\kappa_{x}}\left(N_{x}^{*} \mid \delta_{x}\right)$, where $L_{\kappa_{x}}\left(N_{x}^{*} \mid \delta_{x}\right) \models$ ZFC, and

(iii) knows $A$.

\footnotetext{
${ }^{4}$ The reason we have resorted to a sequence of $J^{\prime} s$ and $Q^{\prime} s$ is that the computation of $\operatorname{HOD}^{L^{J}[x, G]}$, as an iterate of $M_{1}^{J}(x)$ together with a fragment of the canonical strategy for this iterate, seems to require that generic extensions of iterates of $M_{1}^{J}(x)$ know the action of $J$. Since the $Q^{\prime} s$ are not fine-structural, the usual Boolean-valued comparison argument will not work. However, $J_{i+1}$ lets us compute the $C_{\Gamma_{i+1} \text {-closure }}$ operator of $M_{1}^{J}(x)$, which determines easily the $C_{\Gamma_{i+1}}$-closure operator of $M_{1}^{J}(x)[g]$, which then determines $J_{i}$ on $M_{1}^{J}(x)[g]$ via $\mathcal{Q}$-structures.
} 
The notation $N_{x}^{*}$ is somewhat misleading, in that 10.3 gives us different functions $x \mapsto N_{x}^{*}$, depending on which $A \in P_{\beta^{*}+\omega}$ we are trying to capture. We shall make the dependence on $A$ explicit when we need to by

Definition 10.5 Let $x \mapsto\left(M_{x}, N_{x}^{*}, \Sigma_{x}, \delta_{x}\right)$ be as in 10.3 with respect to $A$; then we call the function $x \mapsto\left(M_{x}, N_{x}^{*}, \Sigma_{x}, \delta_{x}\right)$ a coarse mouse operator capturing $A$.

\section{The $L[\vec{E}]$-model $N_{x}$}

Let $x \mapsto N_{x}^{*}$, etc., come from a coarse mouse operator capturing some $A$. We associate an ordinary mouse operator $x \mapsto N_{x}$ by letting $N_{x}$ be the output of the full background extender $K^{c}$-construction over $x$, done inside $N_{x}^{*}$ up to $\delta_{x}$. Since $N_{x}^{*}$ is $\omega_{1}$-iterable in $V$ (where $\mathrm{AD}^{+}$ holds), the full background extender construction does indeed converge to an $x$-premouse $N_{x}$ of height $\delta_{x}$. Note that $N_{x}$ is $\omega_{1}$-iterable in $V$, since trees on it can be lifted to trees on $N_{x}^{*}$. The lifting process itself will be definable in $N_{x}^{*}$, so long as $N_{x}^{*}$ knows how to iterate itself for the lifted trees. This it does know, so long as the lifted trees are in $M_{x}$. So $N_{x}^{*}$ knows how to iterate $N_{x}$, for trees in $M_{x}$.

The following simple lemma implies that $N_{x}$ is a universal weasel in the sense of $N_{x}^{*}$. Its proof is an adaptation of the proof by Mitchell and Schindler in [11] that $K^{c}$ is universal.

Lemma 11.1 Assume ZFC, and let $\delta$ be Woodin. Let $N$ be the output of a maximal full background extender construction of length $\delta$, done over some $x$, and assume this construction does not break down, so that $o(N)=\delta$. Suppose no initial segment of $N$ satisfies "there is a superstrong cardinal". Let $W$ be a premouse over $x$ of height $\leq \delta$, and suppose $\mathcal{P}, \mathcal{Q}$ are the final models above $W, N$ respectively in a successful coiteration. Then $\mathcal{P} \unlhd \mathcal{Q}$.

Proof. Assume instead that $W$ iterates past $N$; that is, that $\mathcal{Q}$ is a proper initial segment of $\mathcal{P}$. Since $\delta$ is inaccessible, we get by standard arguments that $\mathcal{P}=\mathcal{M}_{\delta}^{\mathcal{T}}$ and $\mathcal{Q}=\mathcal{M}_{\delta}^{\mathcal{U}}$, where $(\mathcal{T}, \mathcal{U})$ are the coiteration trees. We also have that $\delta=o(\mathcal{Q})$, while

$$
\delta=i_{\alpha, \delta}^{\mathcal{T}}(\mu)
$$

for some $\alpha$ and $\mu<\delta$, which we now fix. Letting

$$
\kappa_{\beta}=i_{\alpha, \beta}^{\mathcal{T}}(\mu)
$$

we get a club $C \subseteq[0, \delta]_{T} \cap[0, \delta]_{U}$ such that

$$
\beta \in C \Rightarrow \beta=\kappa_{\beta} \wedge i_{0, \delta}^{\mathcal{U}} " \beta \subseteq \beta .
$$

Let us define

$$
f(\gamma)=\text { least } \beta>\gamma \text { such that } \beta \in C \text {. }
$$


Using the Woodiness of $\delta$, we can find a limit point $\kappa$ of $C$, and a $j: V \rightarrow M$ such that

$$
V_{j(f)(\kappa)+\omega} \subseteq M \text { and } j\left(\dot{E}^{N}\right)\left\lceil j(f)(\kappa)=\dot{E}^{N}\lceil j(f)(\kappa) .\right.
$$

Now our backgrounded model $N$ was the output of a maximal construction, so letting $E^{*}$ be the extender of $j$ restricted to $j(f)(\kappa)$, we have that $E=E^{*} \cap N \in N$. We claim that some initial segment of $E$ is of superstrong type in $N$.

For that, it is enough to show that for all $g \in N$ such that $g: \kappa \rightarrow \kappa, i_{E}(g)(\kappa)<j(f)(\kappa)$. For then, $E \nmid \sup \left\{i_{E}(g)(\kappa) \mid g \in N\right\}$ is the desired superstrong initial segment of $E$. So fix $g \in N$. We shall show that in fact, $g(\gamma)<f(\gamma)$ for all sufficiently large $\gamma<\kappa$.

Note that $i_{0, \kappa}(g): \kappa \rightarrow \kappa$, and since $W$ iterated past $N$, we can then find $\eta<\kappa$ such that

$$
i_{0, \kappa}^{\mathcal{U}}(g)=i_{\eta, \kappa}^{\mathcal{T}}(h),
$$

for $h: \kappa_{\eta} \rightarrow \kappa_{\eta}$. Now let $\kappa_{\eta}<\gamma<\kappa$. We then have

$$
\begin{aligned}
g(\gamma) & \leq i_{0, \kappa}^{\mathcal{U}}(g(\gamma))=i_{0, \kappa}^{\mathcal{U}}(g)\left(i_{0, \kappa}^{\mathcal{U}}(\gamma)\right) \\
& =i_{\eta, \kappa}^{\mathcal{T}}(h)\left(i_{0, \kappa}^{\mathcal{U}}(\gamma)\right)<f(\gamma),
\end{aligned}
$$

as desired. The last inequality holds because if $\beta=f(\gamma)$, then $\beta \in C$, so $i_{0, \kappa}^{\mathcal{u}}(\gamma)<\beta$ and $i_{\eta, \kappa}^{\mathcal{T}}(h)\left\lceil\beta=i_{\eta, \beta}^{\mathcal{T}}(h): \beta \rightarrow \beta\right.$.

We can apply 11.1 inside $N_{x}^{*}$, and we get that $N_{x}$ is universal for premice of height $\leq \delta$ there. This is useful, because we have a reasonable fragment of the iteration strategy for $N_{x}$ in $N_{x}^{*}$. In particular, we get

Lemma 11.2 Let $x \mapsto\left(M_{x}, N_{x}^{*}, \Sigma_{x}, \delta_{x}\right)$ be a coarse mouse operator which captures some $A$ such that the complete $\bar{\Gamma}$ set of reals is Wadge reducible to $A$. Let $N_{x}$ be the full bacgroundcertified extender model of $N_{x}^{*} \mid \delta_{x}$. Then for a cone of $x, N_{x}$ is lower part full, in the sense that whenever $\eta$ is a cardinal of $N_{x}$, and $M$ is an $\eta$-sound mouse over $N_{x} \mid \eta$ which projects to $\eta$ and has an $\omega_{1}$-iteration strategy in $\bar{\Gamma}$, then $M \unlhd N_{x}$.

Proof. Because $N_{x}^{*}$ captures $A$, we get both that $M \in N_{x}^{*}$, and that $N_{x}^{*}$ has in it the resriction of the canonical iteration strategy for $M$ to iteration trees in $L\left(N_{x}^{*} \mid \delta_{x}\right)$. But then $N_{x}^{*}$ knows enough of their respective iteration strategies to successfully coiterate $N_{x}$ with $M$. By 11.1, the $N_{x}$ side comes out longer, and this easily implies $M \unlhd N_{x}$.

This is already enough for Woodin's proof that MSC holds in the initial segment of the Wadge hierarchy below iteration strategies for nontame mice, which is properly contained in the minimal model of $\mathrm{AD}^{+}+\neg(\dagger)$. . We give here the proof of Lemma 9.19 under this more restictive assumption; section 14 will show that 9.19 implies the full MSC. 
Theorem 11.3 (Woodin) Assume $\mathrm{AD}^{+}$, and let $\Gamma$ be a good scaled pointclass closed under real quantifiers. Suppose $C_{\Gamma}(x)$ is captured by mice for all $x$; that is, suppose $\mathrm{C}_{\lambda}$ holds, where $\lambda$ is the Wadge ordinal of $\Gamma$. Suppose every $\Gamma$-dual set admits a scale, and let $P_{\beta}=\operatorname{Env}(\Gamma)$ be the envelope of $\Gamma$; then either

(a) for a Turing cone of reals $x$, there is ms-mouse $\mathcal{M}$ over $x$ such that $\mathcal{M}$ has an $\omega_{1}$ iteration strategy in $P_{\beta+2}$, but no $\omega_{1}$ strategy in $\Gamma$, or

(b) there is an $\omega_{1}$ iteration strategy for a nontame ms-mouse in $P_{\beta+2}{ }^{5}$.

Proof. Let $A$ be a universal $\Gamma$ set, and let $x \mapsto\left(M_{x}, N_{x}^{*}, \Sigma_{x}, \delta_{x}\right)$ be a coarse mouse operator capturing $A$. Our construction guarantees that each $N_{x}^{*}$ has an $\omega_{1}$ iteration strategy in $P_{\beta+2}$. Suppose that (a) fails on a cone, and let $x$ be in this cone. It is enough to get a nontame mouse over some set, since then we can rebuild from it a nontame mouse over $\emptyset$.

Claim. For club many $\eta<\delta_{x}, N_{x}^{*} \mid \eta$ is Woodin with respect to all $f: \eta \rightarrow \eta$ such that $f \in C_{\Gamma}\left(N_{x}^{*} \mid \eta\right)$.

Proof. Work in $N_{x}^{*}$, and let $(T, U)$ be our $\delta_{x}^{+}$-absolutely complementing pair capturing $A$. Let $S$ be a transitive model of ZFC ${ }^{-}$with $\left(T, U, N_{x}^{*} \mid \delta_{x}\right) \in S$, and let $\pi: H \rightarrow S$ where $\mathrm{H}$ is transitive, $\left(T, U, N_{x}^{*} \mid \delta_{x}\right) \in \operatorname{ran}(\pi)$, and $\pi(\eta)=\delta_{x}$ for $\eta=\operatorname{crit}(\pi)$. Using the collapses of $T$ and $U$, and the fact that $C_{\Gamma}$-fulllness is $\Gamma$-dual, we get that $C_{\Gamma}\left(N_{x}^{*} \mid \eta\right) \subseteq H$, The elementarity of $\pi$ implies that $N_{x}^{*} \mid \eta$ is Woodin with respect to all $f \in H$, so we have the desired conclusion at $\eta$. Clearly, there are club many such $\eta$.

We may assume that $N_{x}$ has boundedly many Woodin cardinals below $\delta_{x}$, as otherwise $\delta_{x}$ is a Woodin limit of Woodin cardinals in $L\left(N_{x}\right)$, and we have a nontame mouse. (To see that $\delta_{x}$ is Woodin in $L\left(N_{x}\right)$, just note that it is Woodin in $L\left(N_{x}^{*} \mid \delta_{x}\right)$, and $L\left(N_{x}\right)$ is the output of a maximal full background extender construction in $L\left(N_{x}^{*}\right)$.) Now take $\eta$ in the club of our claim, and such that $\eta$ is a cardinal of $N_{x}$, but not Woodin in $N_{x}$.

Let $\mathcal{Q}$ be the largest initial segment of $N_{x}$ satisfying " $\eta$ is Woodin", so that $\mathcal{Q}$ projects to $\eta$, and in fact defines a new $f: \eta \rightarrow \eta$ witnessing $N_{x} \mid \eta$ is not Woodin. If $\eta$ is a cutpoint of $\mathcal{Q}$, then we can regard $\mathcal{Q}$ as a ms-mouse over $N_{x} \mid \eta$, and since we are in the cone where (a) fails, we get that $f$ is in $C_{\Gamma}\left(N_{x} \mid \eta\right)$, and hence $f$ is in $C_{\Gamma}\left(N_{x}^{*} \mid \eta\right)$. By the argument of [12] [§11], there is a $g \in C_{\Gamma}\left(N_{x}^{*} \mid \eta\right)$ witnessing that $N_{x}^{*} \mid \eta$ is not Woodin. ( $g$ is obtained from $f$ and $N_{x} \mid \eta$ in a simple way. Since $\eta$ is a cardinal of $N_{x}, N_{x} \mid \eta$ is the $\eta$-th model of the construction of $N_{x}$, and thus $N_{x} \mid \eta$ is definable over $N_{x}^{*} \mid \eta$.) This contradicts $\eta$ being in our club.

Thus $\eta$ is not a cutpoint of $\mathcal{Q}$, and hence $\mathcal{Q}$ is nontame.

\footnotetext{
${ }^{5}$ One could probably get much better bouunds on the iteration strategies given in (a) and (b); $\Sigma_{4}^{1}(\vec{A})$ for any sjs containing $U$ should suffice.
} 
Corollary 11.4 (Woodin) Assume $\mathrm{AD}^{+}$and $\theta_{0}<\theta$; then there is an $\omega_{1}$-iterable nontame mouse.

Proof. We apply the theorem with $\Gamma=\Sigma_{1}^{2}$. Since $\theta_{0}<\theta$, every $\Gamma$-dual set admits a scale. Clearly, alternative (a) cannot hold, as every iterable mouse has an iteration strategy in $\Gamma$ by the basis theorem. So (b) holds, and we are done.

\section{Two hybrid mouse operators at $\theta_{0}$}

Let $\vec{A}$ be an sjs which seals $\bar{\Gamma}$. We can associate two hybrid mouse operators to $\vec{A}$ as follows.

Definition 12.1 For any countable transitive $b, b^{+}$is the b-mouse obtained by stacking collapsing mice with $\omega_{1}$-iteration strategies in $\bar{\Gamma}$, through $\omega$ cardinals.

Descriptive-set-theoretically, the sets in $b^{+}$are just those in $L[T, b]$ of rank less than the $\omega$-th cardinal above $|b|$ of $L[T, b]$, where $T$ is the tree of a $\bar{\Gamma}$-scale on a universal $\bar{\Gamma}$ set. Our induction hypothesis tells us these are precisely the sets captured by mice with $\bar{\Gamma}$ strategies. Most of the time, $b$ will be selfwellordered, and in fact, mostly it will be a premouse over some real $x$. We write $\mu_{i}\left(b^{+}\right)$for the $i$-th cardinal above $|b|$ in the sense of $b^{+}$.

From [23] we have that for any $A \in \operatorname{Env}(\bar{\Gamma})$, there is a real $x$ such that whenever $x \in b$, and $i<\omega$, there is a term $\tau_{i}^{A}(b)$ capturing $A$ over $b^{+}$at $\mu_{i}\left(b^{+}\right)$. That is, whenever $g$ is generic over $b^{+}$for $\operatorname{Col}\left(\omega, \mu_{i}\left(b^{+}\right)\right)$, then $\tau_{i}^{A}(b)_{g}=A \cap b^{+}[g]$. In fact, this is true whenever $x$ is a $\bar{\Gamma}$-good parameter for $A$. (See 9.13.)

Definition 12.2 Let $b$ be countable and transitive, and let $\vec{A}$ be an sjs which seals $\bar{\Gamma}$, and suppose there is some $x \in b$ which is a $\bar{\Gamma}$-good parameter for $\vec{A}$; then

$$
b \oplus \vec{A}=\left(b^{+}, T\right),
$$

where

$$
T(i, \tau) \Leftrightarrow \tau=\tau_{i}^{A_{(i)_{0}}}(b)
$$

Thus $b \oplus \vec{A}$ is a $b$-mouse with an extra amenable predicate $T$ identifying the sequence of term relations capturing the $A_{i}$ 's at all the $\mu_{j}\left(b^{+}\right)$. Condensation for term relations (see [23, lemma 3.7]) gives

Theorem 12.3 (Woodin) Let $\pi: P \rightarrow b \oplus \vec{A}$ be $\Sigma_{1}$ elementary; then $P=c \oplus \vec{A}$, where $\pi(c)=b$. 
In particular, $b \oplus \vec{A}$ is $\Sigma_{1}$ sound, in the sense that every element is $\Sigma_{1}$ definable from parameters in $b \cup\{b\}$.

Let us fix a real $x_{\vec{A}}$ such that whenever $b$ is countable transitive and $x_{\vec{A}} \in b$, then $b \oplus \vec{A}$ exists. We write

$$
\mathcal{H}_{0}^{\vec{A}}(b)=b \oplus \vec{A}
$$

So 12.3 says that our hybrid mouse operator $\mathcal{H}_{0}^{\vec{A}}$ condenses well.

Let $x \mapsto\left(M_{x}, N_{x}^{*}, \Sigma_{x}, \delta_{x}\right)$ be a coarse mouse operator in $P_{\beta^{*}+\omega}$ which captures $\vec{A}$. Let $N_{x}$ be the full background certified extender model of $N_{x}^{*} \mid \delta_{x}$. We also suppose that there is a $(J, Q)$ as in the proof sketch for 10.3 , so that $M_{x}=M_{1}^{J}(x) \mid \kappa_{x}$ for all $x$, and $J_{i}$ is an iteration strategy with condensation for $Q_{i}$, for all $i<\omega$.

Definition $12.4 \kappa_{\alpha}(x)$ is the $\alpha$-th strong cardinal of $N_{x}$.

Definition 12.5 For $x$ such that $M_{x}$, etc., are defined, we set $\mathcal{H}_{1}^{\vec{A}}(x)=\operatorname{Hull}_{1}^{N_{x} \mid \kappa_{0}(x) \oplus \vec{A}}(x)$.

Thus $\mathcal{H}_{1}^{\vec{A}}(x)$ is the collapse of the set of points a such that for some $i, a$ is first order

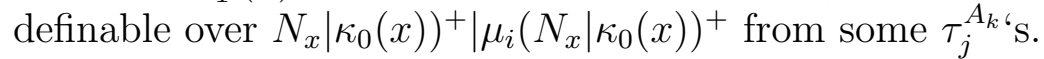

Each of $\mathcal{H}_{0}^{\vec{A}}(x)$ and $\mathcal{H}_{1}^{\vec{A}}(x)$ take one step out of the pure $L[\vec{E}]$ hierarchy and into the $\vec{A}$-hybrid hierarchy, but the transition occurs much later in $\mathcal{H}_{1}^{\vec{A}}(x)$ than it does in $\mathcal{H}_{0}^{\vec{A}}(x)$. Both structures are $\Sigma_{1}$ sound and have $\Sigma_{1}$ projectum $x$, but they are nevertheless not equal, because in effect they represent different indexing conventions.

Let $P \in N_{x}^{*} \mid \delta_{x}$ be transitve, with $x \in P$. Let $R$ be the output of the full background extender construction over $P$, done inside $N_{x}^{*} \mid \delta_{x}$. We shall write

$$
N_{x}^{*} \models W \text { is an } \vec{A} \text {-iterable strong weasel over } P \text {, }
$$

if in $N_{x}^{*}$, there is an iteration map $j: R \rightarrow S$ arising from a finite stack of normal iteration trees played according to the strategy induced by $\Sigma_{x}{ }^{6}$, and an elementary $i: W \rightarrow S$ is such that $i(W \mid \kappa \oplus \vec{A})=S \mid i(\kappa) \oplus \vec{A}$, where $\kappa$ is the least strong cardinal of $W$. This implies $W$ is truly iterable in $V$, via a strategy which moves the term relations for $\vec{A}$ at the least strong cardinal correctly. (It would be more natural to require the term relations at all cardinals be moved correctly, but we do not need that notion.)

In contrast to $\mathcal{H}_{0}^{\vec{A}}(x), \mathcal{H}_{1}^{\vec{A}}(x)$ depends not just on $\vec{A}$ and $x$, but on $N_{x}^{*}$. The next lemma reduces this dependence to some extent.

Lemma 12.6 Let $P \in N_{x}^{*} \mid \delta_{x}$ be transitive, with $x \in P$. Suppose that

$$
N_{x}^{*} \models W \text { is a strong weasel over } P \text {, }
$$

\footnotetext{
${ }^{6}$ It is not hard to generalize 11.1 to this situation, and show that if $o(S)=\delta$, then $S$ is universal in $N_{x}^{*}$, in that no weasel of height $\delta$ iterates past $S$ inside $N_{x}^{*}$.
} 
then

$$
\mathcal{H}_{1}^{\vec{A}}(P)=H u l l_{1}^{W \mid \kappa \oplus \vec{A}}(P),
$$

where $\kappa$ is the least strong cardinal of $W$.

Proof. Let $j: R \rightarrow S$ and $i: W \rightarrow S$ witness that $W$ is an $\vec{A}$-iterable strong weasel in $N_{x}^{*}$. Then

$$
\begin{aligned}
\operatorname{Hull}_{1}^{W \mid \kappa \oplus \vec{A}}(P) & =\operatorname{Hull}_{1}^{S \mid i(\kappa) \oplus \vec{A}}(P) \\
& =\operatorname{Hull}_{1}^{R \mid \mu \oplus \vec{A}}(P)
\end{aligned}
$$

where $\mu=j^{-1}(i(\kappa))$ is the least strong cardinal of $R$.

In $N_{x}^{*}$, we re-build a one-J-Woodin hybrid mouse over $P$, using full background extenders from the $N_{x}^{*}$-sequence with critical points $>o(P)$. We get a proper class model

$$
T \models \text { "I am } M_{1}^{J}(P) "
$$

which is truly iterable (in a way which preserves the $J$-operation). Let $U$ be the output of the maximal full background extender construction over $P$, as done inside $T$. We can compare $U$ with $R$ inside $N_{x}^{*}$, and the proof of 11.1 easily shows that no weasel can iterate past $U$ in $N_{x}^{*}$ (two layers of backgrounding yield as much universality as one), so $U$ vand $R$ have a common iterate via strategies which move the $\vec{A}$-term relations correctly. Thus

$$
\operatorname{Hull}_{1}^{R \mid \mu \oplus \vec{A}}(P)=\operatorname{Hull}_{1}^{U \mid \nu \oplus \vec{A}}(P),
$$

where $\nu$ is the least strong cardinal of $U$.

Now we compare $T$ with the true $M_{1}^{J}(P)$, which was the background universe used to define $N_{P}$, and thence $\mathcal{H}_{1}^{\vec{A}}(P)$. Since we have two $J$-iterable models of "I am $M_{1}^{J}(P)$ ", we get $k(T)=l\left(M_{1}^{J}(P)\right)$, and thence $k(U)=l\left(N_{P}\right)$. The iteration maps $k, l$ move the $\vec{A}$-term relations correctly, and thus

$$
\operatorname{Hull}_{1}^{U \mid \nu \oplus \vec{A}}(P)=\mathcal{H}_{1}^{\vec{A}}(P)
$$

completing the proof.

Remark 12.7 Using lemma 12.6, we can characterize the structure $\mathcal{H}_{1}^{\vec{A}}\left(N_{x} \mid \kappa_{0}(x)\right)$ as follows: let $i: N_{x}^{*} \rightarrow M$ be the ultrapower embedding coming from the background extender for the order zero measure on $\kappa_{0}(x)$ of $N_{x}$. The structure $i\left(N_{x}\right)$ can be regarded as a mouse over $N_{x} \mid \kappa_{0}(x)$. (A mouse over $N_{x} \mid \kappa_{0}(x)$ can have no extenders on its sequence with critical point $\kappa_{0}(x)$. Although $i\left(N_{x}\right)$ has no total measures on its sequence with critical point $\kappa_{0}(x)$, it does have partial measures with this critical point. However, we can inductively "translate" partial measures $\mu$ to $\operatorname{Ult}_{n}(Q, \mu)$, where $Q$ is the longest initial segment of $N_{x}$, and $n$ is as 
large as possible, so that this ultrapower makes sense. Much as with the $*$-transform, this translation gives a level-by-level equivalence between $i\left(N_{x}\right)$ and a mouse $P$ over $N_{x} \mid \kappa_{0}(x)$. To save notation, we also write $i\left(N_{x}\right)$ for $P$.) Then so regarded, we have

$$
\mathcal{H}_{1}^{\vec{A}}\left(N_{x} \mid \kappa_{0}(x)\right)=\operatorname{Hull}_{1}^{i\left(N_{x} \mid \kappa_{0}(x)\right) \oplus \vec{A}}\left(N_{x} \mid \kappa_{0}(x)\right) .
$$

To see this, just apply lemma 12.6, with $W=i\left(N_{x}\right)$ and $P=N_{x} \mid \kappa_{0}(x)$.

We now prove a condensation property for the $\mathcal{H}_{1}^{\vec{A}}$ operator parallel to that proved in 12.3 for the $\mathcal{H}_{0}^{\vec{A}}$ operator. We shall obtain this property by showing that $\mathcal{H}_{0}^{\vec{A}}(P)$ is easily interdefinable with $\mathcal{H}_{1}^{\vec{A}}(P)$, for a cone of $P$. We obtain this equivalence on a cone by using the jump operator comparison techniques of [28]. The proof of this equivalence uses heavily the assumption that the conclusion of Lemma 9.19 fails. (We have not used that assumption yet in this section.) So for the rest of this section, we assume that for a cone of $x$, every ms-mouse over $x$ which has an $\omega_{1}$ strategy in $P_{\beta^{*}+\omega}$ and projects to $x$ has such a strategy in $P_{\alpha^{*}}$.

Let $x_{0} \in \mathbb{R}$ be such that $N_{x}^{*}$, etc., are defined for all reals $x \geq_{T} x_{0}$. Let $x \geq_{T} x_{0}$, and let $k<\omega$. We set

$$
I_{k}(x)=\operatorname{Th}^{\left(x \oplus \vec{A} \mid \mu_{k}(x)\right)}\left(\left\{\tau_{i}^{A_{(i) 0}}(x) \mid i<k\right\}\right),
$$

and

$$
J_{k}(x)=\operatorname{Th}^{\left.P_{x} \oplus \vec{A} \mid \mu_{k}\left(P_{x}\right)\right)}\left(\left\{\tau_{i}^{A_{(i)_{0}}}\left(P_{x}\right) \mid i<k\right\}\right),
$$

where $P_{x}$ is such that $\mathcal{H}_{1}(x)=P_{x} \oplus \vec{A}$. (In other words, $P_{x}$ is $\mathcal{H}_{1}(x)$ cut off at its largest limit cardinal.) It is easy to see that for all $k<\omega$

(1) $I_{k}$ and $J_{k}$ are uniformly Turing invariant functions, and hence jump operators in the sense of [28],

(2) for all $x \geq_{T} x_{0}, I_{k}(x) \leq_{T} J_{k}(x)$,

(3) for any $x \geq_{T} x_{0}, J_{k}(x) \in \mathcal{H}_{1}^{\vec{A}}(x)$, and hence $J_{k}(x) \in C_{\bar{\Gamma}}(x)$, and

(4) for any $x \geq_{T} x_{0}$ and any $y \in C_{\bar{\Gamma}}(x)$, there is a $n$ such that $y \leq_{T} I_{n}(x)$.

Item (4) is a simple consequence of the soundness of $\mathcal{H}_{0}^{\vec{A}}(x)$.

Using (3),(4), and the comparability of jump operators, we get for each $k<\omega$ integers $e_{k}, n_{k}$ and a real $z_{k}$ such that

$$
J_{k}(x)=\left\{e_{k}\right\}^{\left\langle I_{n_{k}}(x), z_{k}\right\rangle},
$$

for all $x \geq_{T} z_{k}$. That is, the jump operator $J_{k}$ is below the jump operator $I_{n_{k}}$ in the Martin order, with $e_{k}$ and $z_{k}$ giving the reduction. Let $z$ be a real coding $x_{0},\left\langle\left(e_{k}, n_{k}\right) \mid k<\omega\right\rangle$, and 
$\left\langle z_{k} \mid k<\omega\right\rangle$. For $x \geq_{T} z, \mathcal{H}_{0}^{\vec{A}}(x)$ is simply coded by the recursive join of the $I_{k}(x)$ for $k<\omega$, while $\mathcal{H}_{1}^{\vec{A}}(x)$ is simply coded by the recursive join of the $J_{k}(x)$ for $k<\omega$, moreover, these two recursive joins are uniformly (granted $z$ ) Turing equivalent to one another.

Henceforth, we write $z_{0}$ for the real parameter $z$ of the last paragraph. Notice now that the definitions of $I_{k}(x)$ and $J_{k}(x)$ make sense for $x$ an arbitrary countable transitive set with $z_{0} \in x$. (In fact, $x_{0} \in x$ is enough.) Let us write $\mathcal{H}_{0, k}^{\vec{A}}(x)$ and $\mathcal{H}_{1, k}^{\vec{A}}(x)$ for the transitive, pointwise definable structures whose theories are $I_{k}(x)$ and $J_{k}(x)$ respectively. Thus for $i=0,1, \mathcal{H}_{i, k}^{\vec{A}}(x) \in x^{+}$for all $k$, and $\mathcal{H}_{i}^{\vec{A}}(x)$ is the direct limit of the $\mathcal{H}_{i, k}^{\vec{A}}(x)$, for $k<\omega$, under the natural maps. Using $z_{0}$ together with condensation for $\mathcal{H}_{0}$, we get a condensation result for $\mathcal{H}_{1}$.

Theorem 12.8 Assume that for a cone of $x$, every ms-mouse over $x$ which has an $\omega_{1}$ strategy in $P_{\beta^{*}+\omega}$ and projects to $x$ has such a strategy in $P_{\alpha^{*}}$. Let $P$ and $Q$ be countable and transitive, with $z_{0} \in P$, and let $\pi: P \rightarrow Q$. The following are equivalent:

(1) $\pi$ extends to a $\Sigma_{1}$ elementary map $\pi^{*}: \mathcal{H}_{0}^{\vec{A}}(P) \rightarrow \mathcal{H}_{0}^{\vec{A}}(Q)$,

(2) $\pi$ extends to a $\Sigma_{1}$ elementary map $\pi^{*}: \mathcal{H}_{1}^{\vec{A}}(P) \rightarrow \mathcal{H}_{1}^{\vec{A}}(Q)$.

Moreover, the map $\pi^{*}$ is unique in each case.

The trick here is just to apply the reductions coded into $z_{0}$ to reals coming from generic enumerations of $P$ and $Q$. We omit further detail.

\section{New mice modulo $(\dagger)$}

In this section we complete the proof of Lemma 9.19. Fix a sjs $\vec{A}$ which seals $\bar{\Gamma}$. Let $y_{0}$ be a real and $\varphi$ a $\Sigma_{n}$ formula in the language of set theory expanded by $\dot{A}$ such that for some $A \in P_{\beta^{*}+1}$, we have $(\mathrm{HC}, \in, A) \models \varphi\left[y_{0}\right]$, but there is no such $A$ in $P_{\beta^{*}}{ }^{7}$

By $(\dagger)$, we have $P_{\beta^{*}+\omega} \subseteq L(\vec{A}, \mathbb{R})$. By the results of [23] relativised to $\vec{A}$, we have that $\Sigma_{1}^{2}$ facts true in $L_{\gamma}(\vec{A}, \mathbb{R})$ are witnessed by hybrid $\vec{A}$-mice with iteration strategies in $L_{\gamma}(\vec{A}, \mathbb{R}) .^{8}$ This gives us a function $r \mapsto M(r)$ which is in $P_{\beta^{*}+3}$, and is defined at all countable transitive $r$ such that $y_{0} \in r$, such that for each countable transitive $r$ in its domain

\footnotetext{
${ }^{7}$ It is easy to prove, using Skolem functions given by scales, that $\varphi$ can be taken to be $\Sigma_{3}$.

${ }^{8} \mathrm{An} \vec{A}$-premouse $\mathcal{M}$ is just like an ordinary ms-premouse, except that if $\mathcal{M} \mid \xi=(\mathcal{M} \mid \gamma)^{+}$, then we must have $(\mathcal{M} \mid \gamma) \oplus \vec{A}$, or in other words $(\mathcal{M} \mid \xi, T)$ for $T$ the term-relation predicate for $\vec{A}$, as the next level of $\mathcal{M}$. We can construct such mice in an appropriate background universe by adding extenders to the sequence as usual, coring down as usual, and adding term-relation predicates when we reach levels of the form $(\mathcal{M} \mid \gamma)^{+}$. We demand of iteration strategies that they move the $\oplus \vec{A}$ operation corrrectly. We can obtain this kind of iterability, and use it to develop a fine structure theory analogous to that of [12], because the $\oplus \vec{A}$ operation condenses well, in the sense of 12.3 .
} 
(a) $M(r)$ is an $\vec{A}$-mouse over $r$,

(b) $M(r)$ has a good $\omega_{1}$-iteration strategy in $P_{\beta^{*}+3}$, and

(c) $o(M(r))=\gamma+\omega$ for some $\gamma$, and for some $\lambda$,

$$
\begin{gathered}
M(r) \mid \gamma \models \lambda \text { is a cardinal, } \\
M(r) \mid \gamma \models \text { there are } n+5 \text { Woodin cardinals }<\lambda,
\end{gathered}
$$

and

$$
M(r) \mid \gamma \models \exists A \in \operatorname{Hom}_{<\lambda}\left((\mathrm{HC}, \in, A) \models \varphi\left[y_{0}\right]\right) .
$$

We assume $M(r)$ is chosen to be the minimal such $\vec{A}$-mouse over $r$, so that $M(r) \mid \gamma$ projects to $r$, where $\gamma$ is as in (c).

Let $\tilde{M}$ be a set of reals coding the function $r \mapsto M(r)$ in some natural way. Now let $\mathcal{N}=\left(x \mapsto\left(M_{x}, N_{x}^{*}, \Sigma_{x}, \delta_{x}\right)\right)$ a coarse mouse operator in $P_{\beta^{*}+3}$ which captures $\vec{A}$ and $\tilde{M}$. From $\vec{A}$ and $\mathcal{N}$ we get the operators $\mathcal{H}_{0}^{\vec{A}}$ and $\mathcal{H}_{1}^{\vec{A}}$ of the last section. Let us assume toward contradiction that for the cone of $x \geq_{T} w_{0}$, every ms-mouse over $x$ which has an $\omega_{1}$ strategy in $P_{\beta^{*}+\omega}$ and projects to $x$ has such a strategy in $P_{\alpha^{*}}$. This gives us a real $z_{0}$ as in the preamble to 12.8. Fix $x \geq_{T} w_{0}, z_{0}$.

Claim 1. There is an $\eta<\delta_{x}$ such that

(a) there is a fully elementary $\pi: M\left(N_{x}^{*} \mid \eta\right) \rightarrow M\left(N_{x}^{*} \mid \delta_{x}\right)$ such that $\eta=\operatorname{crit}(\pi)$ and $\pi(\eta)=$ $\delta_{x}$, and

(b) there is some $B \subseteq \eta$ such that $B \in N_{x}$, but $B \notin M\left(N_{x}^{*} \mid \eta\right)$.

Proof. Work in $N_{x}^{*}$, where we have $M\left(N_{x}^{*} \mid \delta_{x}\right)$ because we have a tree which projects to $\tilde{M}$ in all size $\delta_{x}$ extensions. Using this tree, it is easy to see there are club many $\eta<\delta_{x}$ satisfying (a). ${ }^{9}$

Since $M\left(N_{x}^{*} \mid \delta_{x}\right)$ has cardinality $\delta_{x}$, we have a function $f: \delta_{x} \rightarrow \delta_{x}$ which eventually dominates each $g \in M\left(N_{x}^{*} \mid \delta_{x}\right)$. We can assume $f(\gamma)$ is inacessible, for all $\gamma$. Now let $\eta$ be in the club from (a), and such that $f\left\lceil\eta\right.$ dominates each $g: \eta \rightarrow \eta$ in $M\left(N_{x}^{*} \mid \eta\right)$, and such that there is a $j: V \rightarrow M$ with critical point $\eta$ with $V_{j(f)(\eta)} \subseteq M$ and $j(\vec{E})\lceil j(f)(\eta)=\vec{E}\lceil j(f)(\eta)$, where $\vec{E}$ is the extender sequence of $N_{x}$. We get such a $j$ because $\delta_{x}$ is Woodin. As before, if $f\left\lceil\eta\right.$ eventually dominates all $g: \eta \rightarrow \eta$ in $N_{x}$, then $\eta$ would be Shelah in $N_{x}$, a contradiction. Thus there is a $g: \eta \rightarrow \eta$ which is in $N_{x}$, but not in $M\left(N_{x}^{*} \mid \eta\right)$.

\footnotetext{
${ }^{9}$ One could avoid using the tree by taking $M(r)$ to be $\varphi$-minimal for the appropriate $\varphi$, thereby guaranteeing condensation for the $M$-operator.
} 
Fix $\eta$ as in the claim. We may assume $\kappa<\eta$, where $\kappa=\kappa_{0}(x)$ is the first strong cardinal of $N_{x}$. Let $\gamma$ be least such that there is a subset of $\eta$ definable over $N_{x} \mid \gamma$ which is not in $M\left(N_{x}^{*} \mid \eta\right)$, and set $\mathcal{R}=N_{x} \mid \gamma$. Our plan is to show that $M\left(N_{x}^{*} \mid \eta\right)$ is intertranslatable with some initial segment of $\mathcal{R}\left[N_{x}^{*} \mid \eta\right]$, and thus that some initial segment of $\mathcal{R}$ is a $\left\langle\varphi, y_{0}\right\rangle$-witness. The translation is a variant of the $*$ transform.

Note that $\mathcal{R} \models \eta$ is Woodin. Moreover, the total extenders from the $\mathcal{R} \mid \eta$ sequence whose support is a cardinal of $\mathcal{R}$ extend to extenders over $N_{x}^{*}$ with strength $\geq$ this support. It follows that $N_{x}^{*} \mid \eta$ is generic over $\mathcal{R}$ for the $\eta$-generator extender algebra of $\mathcal{R}$ at $\eta$. Thus $\mathcal{R}\left[N_{x}^{*} \mid \eta\right]$ makes sense. It is not clear that we can regard it as a mouse over $N_{x}^{*} \mid \eta$, however, because $\eta$ may not be a cutpoint of $\mathcal{R}$. Our smallness assumption $(\dagger)$ does give

Claim 2. Let $E$ be an extender on the $\mathcal{R}$ sequence such that $\operatorname{crit}(E) \leq \eta \leq \operatorname{lh}(E)$; then $\operatorname{crit}(E)=\kappa$.

Proof. Since $\kappa$ is the least strong, it is a limit of cutpoints on the $N_{x}$-sequence, so $\kappa \leq \operatorname{crit}(E)$. Since $\eta$ is Woodin in $\mathcal{R}$ and by $(\dagger)$ there is no iterable mouse with a measurable Woodin, $\operatorname{crit}(E)<\eta$. Also, $\eta$ is Woodin in $\mathcal{R}$, and hence Woodin in $\operatorname{Ult}(\mathcal{R}, E)$, and thus $\operatorname{crit}(E)$ is a limit of Woodins in $\mathcal{R}$. If $\kappa<\operatorname{crit}(E)$, then $\kappa$ is $<\operatorname{crit}(E)$-strong in $\mathcal{R}$. Thus the minimal active mouse satisfying "there is a $\lambda$ which is a limit of Woodins and such that some $\kappa<\lambda$ is $<\lambda$-strong" is an initial segment of $\mathcal{R}$, and hence has an $\omega_{1}$ iteration strategy. This contradicts $(\dagger)$.

Let us write $g=N_{x}^{*} \mid \eta$. We now define an analog of the $*$-transform. Our analog associates, to initial segments $\mathcal{Q}[g]$ of $\mathcal{R}[g], \vec{A}$-mice $\mathcal{Q}[g]^{a}$ over $g$, in such a way that $\mathcal{Q}[g]$ and $\mathcal{Q}[g]^{a}$ are intertranslatable.

To begin with, letting $\alpha>\eta$ be least such that $\mathcal{R} \mid \alpha \models \mathrm{KP}$, there is clearly a unique mouse $\mathcal{S}$ over $g$ such that $\mathcal{R} \mid \alpha[g]={ }^{*} \mathcal{S}$, and we let $(\mathcal{R} \mid \alpha[g])^{a}$ be this unique $\mathcal{S}$.

Let $U$ be the tree of all finite sequences $\left\langle E_{0}, \ldots, E_{n}\right\rangle$ such that each $E_{i}$ is an extender with $\operatorname{crit}\left(E_{i}\right)=\kappa$ and $\operatorname{lh}\left(E_{i}\right) \geq \eta$, and $E_{0}$ is on the $\mathcal{R}$-sequence, and $E_{i+1}$ is on the sequence of $\operatorname{Ult}\left(N_{x}, E_{i}\right)$ for all $i<n$. For $\vec{E}=\left\langle E_{0}, \ldots, E_{n}\right\rangle$ in $U$, we write $\mathcal{P}(\vec{E})$ for $\operatorname{Ult}\left(N_{x}, E_{n}\right)$. Here we understand that $\mathcal{P}(\emptyset)=\mathcal{R}$. Because $N_{x}$ is iterable, $U$ is wellfounded. We shall define $\mathcal{Q}[g]^{a}$ for all $\mathcal{Q} \unlhd \mathcal{P}(\vec{E})$ where $\vec{E} \in U$ such that $o(\mathcal{Q})$ is at least the first admissible over $\mathcal{R} \mid \eta$. The definition is by induction on the $U$-rank of $\vec{E}$, with a subinduction on $o(\mathcal{Q})$.

The inductive clauses are as follows:

(a) if $o(\mathcal{Q})=\omega \alpha+\omega$, then $\mathcal{Q}[g]^{a}$ is obtained from $\mathcal{Q} \mid \alpha[g]^{a}$ by taking one step in the $J$-hierarchy.

(b) if $o(\mathcal{Q})$ is a limit ordinal and $\mathcal{Q}$ is passive, then $\mathcal{Q}[g]^{a}=\bigcup\left\{(\mathcal{Q} \mid \eta)[g]^{a} \mid \eta<o(\mathcal{Q})\right\}$.

(c) if $\mathcal{Q}$ is active with last extender $E$, and $\operatorname{crit}(E)>\eta$, then letting $\mathcal{Q}=(\mathcal{S}, E)$, we set $\mathcal{Q}[g]^{a}=\left(\mathcal{S}[g]^{a}, E\right)$. 
(d) if $\mathcal{Q}$ is active with last extender $E$, and $\operatorname{crit}(E)=\kappa$, then $\mathcal{Q}[g]^{a}=\left(\operatorname{Ult}\left(N_{x}, E\right) \mid \gamma\right)[g]^{a} \oplus$ $\vec{A}$, where $\gamma$ is the least cardinal cutpoint $\xi$ of $\operatorname{Ult}\left(N_{x}, E\right)$ such that $\nu(E) \leq \xi$.

Note that the ultrapower in case (d) makes sense, and that the least $\xi$ as in (d) is $<i_{E}(\kappa)$. Note also that in case (d), we have that $\left.\operatorname{Ult}\left(N_{x}, E\right) \mid \gamma\right)^{+} \unlhd \operatorname{Ult}\left(N_{x}, E\right)$, so we could also write $\left.\mathcal{Q}[g]^{a}=\left(\operatorname{Ult}\left(N_{x}, E\right) \mid \gamma\right)^{+}[g]^{a}, T\right)$, where $T$ is the term relation predicate for $\vec{A}$.

Again, the detailed verification that $\mathcal{Q}[g]$ and $\mathcal{Q}[g]^{a}$ are intertranslatable takes some work. The basic idea is as follows. Since $N_{x} \mid \kappa \oplus \vec{A} \in g, \mathcal{Q}[g]$ can recover $\mathcal{Q}[g]^{a}$ by employing the inductive definition we just gave. ( $N_{x}$ has the same subsets of $\kappa$ as does $N_{x} \mid \kappa \oplus \vec{A}$, because $\kappa$ is a cutpoint of $N_{x}$, and we are in the cone where 9.19 fails. The embedding associated to $\operatorname{Ult}\left(N_{x} \mid \kappa \oplus \vec{A}, E\right)$ moves the term relations for $\vec{A}$ correctly, and these image term relations can be used to compute the $\oplus \vec{A}$ part of $\left(\operatorname{Ult}\left(N_{x} \mid \kappa \oplus \vec{A}, E\right) \mid \gamma\right)[g]^{a} \oplus \vec{A}$.) Conversely, if we want to recover $\mathcal{Q}[g]$ from $\mathcal{Q}[g]^{a}$, all is trivial unless inductive clause (d) applies.

We sketch how to do the recovery in case (d). Adopting the notation there, we wish to recover $E$ from $\operatorname{Ult}\left(N_{x}, E\right) \mid \gamma[g]^{a} \oplus \vec{A}$. We can recover $\operatorname{Ult}\left(N_{x}, E\right) \mid \gamma$ by induction. Since $z_{0} \leq_{T} x$, we can then use $\operatorname{Ult}\left(N_{x}, E\right) \mid \gamma \oplus \vec{A}$ to obtain $\mathcal{H}_{1}^{\vec{A}}\left(\operatorname{Ult}\left(N_{x}, E\right) \mid \gamma\right)$. We can regard $\operatorname{Ult}\left(N_{x}, E\right)$ as a weasel over $\operatorname{Ult}\left(N_{x}, E\right) \mid \gamma$, and we have then that

$$
N_{x}^{*} \models \operatorname{Ult}\left(N_{x}, E\right) \text { is an } \vec{A} \text {-iterable strong weasel over } \operatorname{Ult}\left(N_{x}, E\right) \mid \gamma \text {. }
$$

So by Lemma 12.6 , there is a $\Sigma_{1}$-elementary

$$
\pi: \mathcal{H}_{1}^{\vec{A}}\left(\mathrm{Ult}\left(N_{x}, E\right) \mid \gamma\right) \rightarrow i_{E}\left(N_{x} \mid \kappa \oplus \vec{A}\right),
$$

such that $\pi \mid \gamma=$ identity. (Here we regard $i_{E}\left(N_{x} \mid \kappa \oplus \vec{A}\right)$ as a mouse over $\operatorname{Ult}\left(N_{x}, E\right) \mid \gamma$.) We have $i_{E}$ " $\left(N_{x} \mid \kappa \oplus \vec{A}\right) \subseteq \operatorname{ran}(\pi)$, because $\left(N_{x} \mid \kappa \oplus \vec{A}\right)$ is the $\Sigma_{1}$ hull of $\kappa$. Thus setting

$$
j=\pi^{-1} \circ i_{E} \uparrow\left(N_{x} \mid \kappa \oplus \vec{A}\right),
$$

we have

$$
j: N_{x} \mid \kappa \oplus \vec{A} \rightarrow \mathcal{H}_{1}^{\vec{A}}\left(\operatorname{Ult}\left(N_{x}, E\right) \mid \gamma \oplus \vec{A}\right) .
$$

Now we can recover $j$ from $N_{x} \mid \kappa \oplus \vec{A}$ and $\mathcal{H}_{1}^{\vec{A}}\left(\operatorname{Ult}\left(N_{x}, E\right) \mid \gamma \oplus \vec{A}\right)$ ), which we have available, as the uncollapse embedding associated to the $\Sigma_{1}$ hull of $N_{x} \mid \kappa$. Since $\pi\lceil\gamma=$ identity, the extender of $j$ agrees with that of $i_{E}$ out to $\gamma$. Since $\gamma \geq \nu(E)$, we can thus recover $E$ from $j$. This ends our sketch of the intertranslatability of $\mathcal{Q}[g]$ and $\mathcal{Q}[g]^{a}$, for $\mathcal{Q} \unlhd \mathcal{R}$.

Remark 13.1 There is a fine point here. In order to say that $\mathcal{Q}[g]^{a}$ is always a level of an $\vec{A}$-mouse, we must understand "level" in such a way that $\xi+1$-st level of an $\vec{A}$-mouse $\mathcal{M}$ is not $\mathcal{M} \mid \xi \oplus \vec{A}$, but rather the first level of $(\mathcal{M} \mid \xi)^{+}$. The $\vec{A}$-active levels of $\mathcal{R}[g]^{a}$ come exactly from translating extenders with critical point $\kappa$, as in case (d). The following easily proved 
fact tells us how often this happens: let $\vec{E} \in U$, let $N=\mathcal{P}(\vec{E})$, and let $\mu, \nu$ be indices of extenders on the $N$-sequence with critical point $\kappa$, with $\nu$ the least such index $>\mu$. Then $N\left|\nu=(N \mid \mu)^{+}\right| \nu$, and $\nu$ is the least cardinal $>\mu$ of $(N \mid \mu)^{+}$.

Now $\mathcal{R}$ defines a subset of $\eta$ not in $M\left(N_{x}^{*} \mid \eta\right)$, and thus $\mathcal{R}[g]^{a}$ cannot be a proper initial segment of $M\left(N_{x}^{*} \mid \eta\right)$. It follows that, setting $\gamma+\omega=o\left(M\left(N_{x}^{*} \mid \eta\right)\right), M\left(N_{x}^{*} \mid \eta\right) \mid \gamma$ is a proper initial segment of $\mathcal{R}[g]^{a}$. Let $\lambda$ be such that for $r=N_{x}^{*} \mid \eta$,

$$
\begin{gathered}
M(r) \mid \gamma \models \lambda \text { is a cardinal, } \\
M(r) \mid \gamma \models \text { there are } n+5 \text { Woodin cardinals }<\lambda,
\end{gathered}
$$

and

$$
M(r) \mid \gamma \models \exists A \in \operatorname{Hom}_{<\lambda}\left((\mathrm{HC}, \in, A) \models \varphi\left[y_{0}\right]\right) .
$$

Inspecting our translation procedure, one sees that because $\lambda$ is a limit of $\vec{A}$-active levels of $M(r), M(r) \mid \lambda=(\mathcal{R} \mid \lambda)[g]^{a}$, and $(\mathcal{R} \mid \lambda)[g]$ has the same universe as $(\mathcal{R} \mid \lambda)[g]^{a}$. Thus $\mathcal{R} \mid(\lambda)[g]$ has an $n+5$-th Woodin cardinal $\delta>\eta$, and

$$
(\mathcal{R} \mid \lambda)[g] \models \exists A \in \operatorname{Hom}_{\delta^{+}}\left((\mathrm{HC}, \in, A) \models \varphi\left[y_{0}\right]\right) .
$$

A result of Woodin (cf. [25, Theorem 5.1]) shows that then

$$
\mathcal{R} \mid \lambda \models \exists A \in \operatorname{Hom}_{\delta^{+}}\left((\mathrm{HC}, \in, A) \models \varphi\left[y_{0}\right]\right) .
$$

Thus $\mathcal{R} \mid \lambda$ is a $\left\langle\varphi, y_{0}\right\rangle$-witness. The first initial segment of $\mathcal{R}$ which is such a witness is easily seen to project to $\omega$, and have no $\omega_{1}$-iteration strategy in $P_{\beta^{*}}$. This is a contradiction, completing the proof of Lemma 9.19.

\section{Proof of $S_{\beta^{*}+\omega}$.}

In this section, we prove

Theorem 14.1 Assume $\mathrm{AD}^{+}$, and let $[\alpha, \beta]$ be a $\Sigma_{1}^{2}$ gap, with $\alpha<\beta$. Suppose $S_{\alpha}$ holds. Suppose also there is a function $J$ defined on a Turing cone of reals $y$, with $J$ coded by a set of reals in $P_{\beta+\omega}$, and such that for $y \in \operatorname{dom}(J)$, we have $J(y)=\left(\mathcal{S}_{y}, \Omega_{y}\right)$ where

(a) $\mathcal{S}_{y}$ is a ms-mouse over $y$ which projects to $\omega$,

(b) $\Omega_{y}$ is an $\omega_{1}$-iteration strategy for $\mathcal{S}_{y}$, and

(c) $\mathcal{S}_{y}$ has no $\omega_{1}$-iteration strategy in $P_{\beta}$. 


\section{Then $S_{\beta+\omega}$ holds.}

By 9.19, we have that $(\dagger)$ implies there is a $J$ as in the hypotheses of the theorem above when $\alpha=\alpha^{*}$ and $\beta=\beta^{*}$. So a proof of 14.1 will complete our proof of $S_{\beta^{*}+\omega}$ under the smallness assumption $(\dagger)$. It is worth emphasizing, however, that there is no smallness assumption in 14.1.

Proof of 14.1. We first derive a strengthened form of the hypotheses of 14.1.

Lemma 14.2 Assume the hypotheses of 14.1, and let $\Gamma$ be a good scaled pointclass such that $\Gamma \subseteq P_{\beta+\omega}$. Then for a Turing cone of reals $y$, there is an ms-mouse $\mathcal{M}$ over $y$ such that

(a) $\mathcal{M}_{y}$ projects to $\omega$,

(b) $\mathcal{M}_{y}$ has an $\omega_{1}$-iteration strategy in $P_{\beta+\omega}$, but

(c) $\mathcal{M}_{y}$ is not coded by a real in $C_{\Gamma}(y)$.

Proof. Let $J$ be as in the hypothesis of 14.1. We may assume that $J$ is coded by a set of reals in $\Gamma$. Let $x \mapsto\left(M_{x}, N_{x}^{*}, \Sigma_{x}, \delta_{x}\right)$ be a coarse mouse operator capturing $U$, where $U$ is a universal $\Gamma$ set. We can assume that the iteration strategies $\Sigma_{x}$ (and for that matter, the whole operator) are in $P_{\beta+\omega}$. Fix any real $x$ in the domain of this operator (which is a Turing cone), and in the cone where $J$ is defined. It is enough to find a real $y \geq_{T} x$ and a mouse $\mathcal{M}_{y}$ over $y$ such that the conclusion of 14.2 holds.

Claim 1. $N_{x} \models$ there are finitely many Woodin cardinals.

Proof. Suppose not, and let $\lambda$ be a limit of Woodin cardinals in $N_{x}$. Because we are in the cone where $J$ is defined, $\mathcal{S}_{x}$ is a mouse over $x$ whose unique $\omega_{1}$-iteration strategy $\Omega_{x}$ is in $\Gamma$, but not in $P_{\beta}$. Now $N_{x}^{*}$ is sufficiently correct that

$$
N_{x}^{*} \models \mathcal{S}_{x} \text { is } \delta_{x}+1 \text { iterable above } \delta .
$$

By 11.2, we have that $\mathcal{S}_{x} \unlhd N_{x}$.

Going further in that direction, let $\gamma<\delta_{x}$, and let $W$ be the result of a full background construction over $x$ done in $N_{x}$ with critical points above $\gamma$. It is not hard to modify the proof of 11.1 to show that $N_{x}^{*} \models W$ is universal, and thus that $\mathcal{S}_{x} \unlhd W$.

By the last paragraph, we can compute $\Omega_{x}\left\lceil N_{x}\right.$ inside $N_{x}$ by reducing iterations of $\mathcal{S}_{x}$ to iterations of $N_{x}$ above sufficiently large $\gamma$, using UBH in $N_{x}$ to compute the correct branches for trees of size $<\gamma$. (See [21] for an expansion of this point.) This yields definable class trees $T$ and $T^{*}$ which project to a set $\tilde{\Omega}$ of reals coding $\Omega_{x}$, and to $\mathbb{R} \backslash \tilde{\Omega}$, in all size $<\delta_{x}$ extensions of $N_{x}$. Moreover, if $j: N_{x} \rightarrow M$ comes from an iteration following the strategy 
induced by $\Sigma_{x}, j(T)$ and $j\left(T^{*}\right)$ still project to $\tilde{\Omega}$ and its complement in set-generic extensions of $M$.

Now let $I$ be an $\mathbb{R}$-genericity iteration of $N_{x}$ below $\lambda$, using the strategy induced by $\Sigma_{x}$, and let $D$ be the resulting derived model. We have just shown $\Omega \in D$, and hence $P_{\beta+\omega} \subseteq D$. We may assume that $x$ was chosen large enough that there is a real $z$ such that $z \in \mathrm{OD}^{D}(x)$, but $z \notin \mathrm{OD}^{P_{\beta+\omega}}(x)$. (For a cone of $x$ there is such a $z$, because the pointclass $\left(\Sigma_{1}^{\mathbf{2}}\right)^{\mathbf{D}}$ properly includes $P_{\beta+\omega}$, because our gap ended, and thus we can uniformize $z \notin \operatorname{OD}^{\beta+\omega}(x)$ by a $\left(\Sigma_{1}^{\mathbf{2}}\right)^{\mathbf{D}}$ function.) But since $z \in \mathrm{OD}^{D}(x)$, we get $z \in N_{x}$, and since $N_{x}$ has an iteration strategy in $P_{\beta+\omega}$, this implies $z \in \mathrm{OD}^{P_{\beta+\omega}}(x)$, a contradiction.

Working in $N_{x}^{*}$, we can find club many $\eta<\delta_{x}$ such that

(a) $\eta$ is a cardinal, and $N_{x} \mid \eta$ is the $\eta$-th model of the $N_{x}$-construction, and

(b) $\eta$ is $\Gamma$-Woodin cardinal, in that whenever $f: \eta \rightarrow \eta$ and $f \in C_{\Gamma}\left(N_{x}^{*} \mid \eta\right)$, then there is an extender in $N_{x}^{*} \mid \eta$ witnessing the Woodin property with respect to $f$.

(Proof: Let $T, T^{*}$ be trees witnessing that $U$ is captured vis-a-vis the collapse of $\delta_{x}$. Let $M \models$ ZFC $^{-}$with $T, T^{*} \in M$, and let $\pi: H \rightarrow M$ with $T, T^{*} \in \operatorname{ran}(\pi)$, and $\pi(\eta)=\delta_{x}$, for $\eta=\operatorname{crit}(\pi)$. By arguments we have given already, $\eta$ is $\Gamma$-Woodin.)

By claim 1, we can fix an $\eta$ having properties (a) and (b) such that $\eta$ is not Woodin in $N_{x}$. Let $\mathcal{Q}=N_{x} \mid \gamma$, where $\gamma$ is least such that there is a $f: \eta \rightarrow \eta$ such that no extender from $N_{x} \mid \eta$ witnesses the Woodin property with respect to $f$, with $f$ definable over $N_{x} \mid \gamma$. Note that there is no extender $E$ on the $\mathcal{Q}$ sequence such that $\operatorname{crit}(E) \leq \eta \leq \operatorname{lh}(E)$, for otherwise, $\operatorname{crit}(E)$ would be a cardinal in $N_{x}$ because $\eta$ is, and then $\operatorname{crit}(E)$ would be a limit of Woodin cardinals in $N_{x}$, contrary to claim 1 . Thus we can consider $\mathcal{Q}$ as a mouse over $N_{x} \mid \eta$. By (a), $N_{x} \mid \eta$ is first order definable over $N_{x}^{*} \mid \eta$, and by (b) then, $\mathcal{Q} \notin C_{\Gamma}\left(N_{x} \mid \eta\right)$. Let $g$ be $\operatorname{Col}(\omega, \eta)$-generic over $L\left[T, N_{x} \mid \eta\right]$, where $T$ is the tree of a $\Gamma$-scale on $U$, and let $y$ be a real naturally coding $\left\langle N_{x} \mid \eta, g\right\rangle$. Then $\mathcal{Q}[g]$ can be regarded as a mouse $\mathcal{M}_{y}$ over $y$, and it is easy to see that the conclusions of 14.2 hold for $\mathcal{M}_{y}$.

The comparability of inner model operators gives

Corollary 14.3 Let $\Gamma \subseteq P_{\beta+\omega}$ be a good scaled pointclass; then there is a mouse operator $J_{\Gamma}$ coded by a set of reals in $P_{\beta+\omega}$, and such that for all $a \in \operatorname{dom}\left(J_{\Gamma}\right)$

1. $J_{\Gamma}(a)=\left(\mathcal{M}_{a}, \Omega_{a}\right)$, where $\mathcal{M}_{a}$ is an ms-mouse over a which projects to a, and has (unique) $\omega_{1}$ iteration strategy $\Omega_{a}$, and

2. $C_{\Gamma}(a) \subseteq \mathcal{M}_{a}$. 
Proof. By 14.2 we get a real $z$ and a mouse jump operator $J$ defined on all reals $a \geq_{T} z$ having property (1), and (2) weakened to " $\mathbb{R} \cap \mathcal{M}_{a} \nsubseteq C_{\Gamma}(a)$ ". ( $J$ just picks the least mouse over $a$ not in $C_{\Gamma}(a)$. Clearly $J$ is uniformly Turing invariant.) Using [28], we see that $J$ satisfies the full (2) on the cone of all $a \geq_{T} x$, for some $x \geq_{T} z$. Finally, we can extend $J$ to the desired $J_{\Gamma}$ acting on all countable transitive $a$ such that $x \in a$ by Lemma 4.5.

We now complete the proof of $\mathrm{S}_{\beta+\omega}$. Let $y$ be a real and $A \in P_{\beta+\omega}$, and suppose

$$
(\mathrm{HC}, \in, A) \models \varphi[y] .
$$

We seek a $\langle\varphi, y\rangle$-witness $(\mathcal{M}, \Sigma)$ such that $\Sigma \in P_{\beta+\omega}$. Suppose that $\varphi$ is $\Sigma_{n}$.

Let $k=n+10$, and let $\left\langle\Gamma_{i} \mid i \leq k\right\rangle$ be a strictly increasing sequence of good scaled pointclasses, with $A \in \Gamma_{0}$, and $\Gamma_{k} \subseteq P_{\beta+\omega}$. Let $J=J_{\Gamma_{0}}$ be as in 14.3 for $\Gamma_{0}$, with its domain being the HC-cone with base $x_{0}$. We assume $\Gamma_{1}$ is chosen large enough that there is a set of reals in $\Gamma_{1}$ which codes $J$. Let $U_{i}$ be a universal $\Gamma_{i}$ set, and let $x \mapsto\left(M_{x}, N_{x}^{*}, \Sigma_{x}, \delta_{x}\right)$ be a coarse mouse operator in $P_{\beta+\omega}$ which captures $U_{k}$. Fix a real $x$ in the domain of this operator, and such that $y, x_{0} \leq_{T} x$.

Working in $N_{x}^{*}$, let $\left\langle\mathcal{N}_{\xi} \mid \xi<\delta_{x}\right\rangle$ be the stages of the full background extender construction done over $y$ through $\delta_{x}$ stages. We shall show that some $\mathcal{N}_{\xi}$ is a $\langle\varphi, y\rangle$-witness.

For $i \leq k$, we have trees $T_{i}, T_{i}^{*}$ such that whenever $j: N_{x}^{*} \rightarrow S$ comes from an iteration via $\Sigma_{x}$, and $g$ is $S$-generic over $\operatorname{Col}\left(\omega, \delta_{x}\right)$, then

$$
U_{i} \cap S[g]=p\left[j\left(T_{i}\right)\right] \cap S[g]
$$

and

$$
\left(\mathbb{R} \backslash U_{i}\right) \cap S[g]=p\left[j\left(T_{i}^{*}\right)\right] \cap S[g] .
$$

Working in $N_{x}^{*}$, let us call $\eta$ a $\Gamma_{i}$-Woodin cardinal iff by using $\left(T_{i}, T_{i}^{*}\right)$ to compute $C_{\Gamma}\left(N_{x}^{*} \mid \eta\right)$, we see that $\eta$ is Woodin with respect to all functions in $C_{\Gamma}\left(N_{x}^{*} \mid \eta\right)$. Let us put

$$
\eta_{k}=\text { the least } \Gamma_{k} \text {-Woodin cardinal, }
$$

and for $1 \leq i \leq k$,

$$
\eta_{i-1}=\text { the least } \Gamma_{i-1} \text {-Woodin cardinal }>\eta_{i} .
$$

So $\eta_{k}<\eta_{k-1}<\ldots<\eta_{0}$, and $\eta_{i}$ is $\Gamma_{i}$-Woodin. Let $W=\mathcal{N}_{\eta_{0}}$. We shall show that $W$ is a $\langle\varphi, y\rangle$-witness.

Claim 1. Let $\eta=\eta_{i}$, where $1 \leq i \leq k$; then $\mathcal{N}_{\eta}=W \mid \eta, \eta$ is a cutpoint of $W$, and $\eta$ is Woodin in $W$.

Proof. Because $\eta$ is the first $\Gamma_{i}$-Woodin above some point, $\eta$ is not measurable in $N_{x}^{*}$, and there is in $N_{x}^{*}$ no $j: V \rightarrow M$ with $\operatorname{crit}(j)<\eta$ and $N_{x}^{*} \mid \eta \subseteq M$. (Proof: let $m$ be largest such 
that $\operatorname{crit}(j) \leq \eta_{m}$. Then $\eta_{m}$ is $\Gamma_{m}$-Woodin in $N_{x}^{*}$ as certified by $T_{m}, T_{m}^{*}$, so $\eta_{m}$ is $\Gamma_{m}$-Woodin in $M$ as cerified by $j\left(T_{m}\right), j\left(T_{m}^{*}\right)$, by a standard argument. We can then pull back to get that $\operatorname{crit}(j)$ is a limit of $\Gamma_{m}$-Woodins, as certified by $T_{m}, T_{m}^{*}$. But $\operatorname{crit}(j)>\eta_{m+1}$ if $m<k$, so in any case, we have a contradiction.))

Now let $\mathcal{R}=\mathcal{N}_{\eta}$. It will be enough to show that if $\eta<\xi<\eta_{0}$, then $\rho_{\omega}\left(\mathcal{N}_{\xi}\right) \geq \eta$ and for every $f: \eta \rightarrow \eta$ definable over the core of $\mathcal{N}_{\xi}$, some extender from the $\mathcal{R}$-sequence witnesses the Woodin property with respect to $f$. So suppose not, and let $\mathcal{Q}=\mathcal{N}_{\xi}$ where $\xi$ is the least counterexample. By the remarks of the last paragraph, $\eta$ is a cutpoint of $\mathcal{Q}$.

Clearly $\rho_{\omega}(\mathcal{Q}) \leq \eta$. Let $\mathcal{S}$ be the $\eta$-core of $\mathcal{Q}$, that is, the transitive collapse of the $\Sigma_{n}$-hull of $\eta$ in $\mathcal{Q}$, where $n$ is least such that $\rho_{n}(\mathcal{Q}) \leq \eta$. We can regard $\mathcal{S}$ as a sound mouse over $\mathcal{R}=\mathcal{S} \mid \eta$. $\mathcal{S}$ has an iteration strategy easily computed from $\tau$, where $\tau$ is the restriction of $\Sigma_{x}$ to trees based on $N_{x}^{*} \mid \eta_{0}$ which are above $\eta=\eta_{i}$. But at worst we have $\Gamma_{i-1}$-Woodins in this interval, so $\Omega$ is coded by a set of reals in $\Gamma_{i}$, and in fact, $\mathcal{S}$ is coded by a subset of $\eta$ in $C_{\Gamma_{i}}(\mathcal{R})$.

Because $\eta$ is $\Gamma_{i}$-Woodin, $\mathcal{S}$ cannot define a bad $f: \eta \rightarrow \eta$. So let $\rho<\eta$ and $B \subseteq \rho$, with $B$ definable over $S$ but not in $\mathcal{R}$. Let $T$ be the tree of a $\Gamma_{i}$ scale on $U_{i}$. We work in $L\left(T, N_{x}^{*} \mid \eta\right)$, which satisfies that $\eta$ is Woodin. Note $\mathcal{S} \in L\left(T, N_{x}^{*} \mid \eta\right)$. Let $M=L_{\gamma}\left(T, N_{x}^{*} \mid \eta\right) \models \mathrm{ZFC}^{-}$, and let

$$
\pi: H \rightarrow M
$$

with $\rho<\bar{\eta}=\operatorname{crit}(\pi)<\eta$, and

$$
\pi((\overline{\mathcal{S}}, \bar{\eta}))=(\mathcal{S}, \eta)
$$

We have that $\bar{\eta}$ is a cutpoint of $\overline{\mathcal{S}}, \overline{\mathcal{S}}|\bar{\eta}=\mathcal{R}| \bar{\eta}$, and $B$ is definable over $\overline{\mathcal{S}}$.

Working now in $V$, let us compare $\overline{\mathcal{S}}$ with $\mathcal{R}$. We do this as in the proof of 12.6 , moving the model on the $\mathcal{R}$ side of the coiteration by the background extender embeddings. This gives us iteration trees $\mathcal{T}$ on $\overline{\mathcal{S}}$ with last model $\mathcal{P}$, and $\mathcal{U}$ on $N_{x}^{*}$ with embedding $j: N_{x}^{*} \rightarrow Z$, such that $j(\mathcal{R}) \unlhd \mathcal{P}$. It is not hard to see that $\overline{\mathcal{S}} \in j\left(N_{x}^{*} \mid \eta\right)$. Let us now work in $L\left(\left[j(T), j\left(N_{x}^{*} \mid \eta\right)\right]\right.$. Since $\mathcal{S}$ has an iteration strategy in $\Gamma_{i}$ for trees above $\eta$, so does $\overline{\mathcal{S}}$ for trees above $\bar{\eta}$. The strategy is unique, and using $j(T)$ we can compute it. Thus if we compare $\overline{\mathcal{S}}$ with $j(\mathcal{R})$ in $L\left(\left[j(T), j\left(N_{x}^{*} \mid \eta\right)\right], \overline{\mathcal{S}}\right.$ iterates via $\mathcal{T}$ to $\mathcal{P}$, while $j(\mathcal{R})$ does not move. It is easy to use the proof of our lemma on universality at Woodin cardinals (11.1) in $L\left(\left[j(T), j\left(N_{x}^{*} \mid \eta\right)\right]\right.$, where $j(\eta)$ is Woodin, to get a contradiction.

By our claim, $x$ is generic over $W$ for an extender algebra at $\eta_{k}$. Moreover, we can regard $W[x]$ as a mouse over $\left\langle W \mid \eta_{k}, x\right\rangle$.

Claim 2. $C_{\Gamma_{0}}\left(W[x] \mid \eta_{1}\right) \subseteq W[x]$.

Proof. Consider $J\left(W[x] \mid \eta_{1}\right)=(\mathcal{M}, \Omega)$. It will be enough to show $\mathcal{M} \unlhd W[x]$, because $a=W[x] \mid \eta_{0}$ has $x$ in it, so that (2) of 14.3 applies. So suppose not. Working in $V$, we compare $\mathcal{M}$ with $W[x]$. We do this as in [17], moving the model on the $W[x]$ side of the 
coiteration by the background extender embeddings. This gives us iteration trees $\mathcal{T}$ on $\mathcal{M}$ by $\Omega$ with last model $\mathcal{P}$, and $\mathcal{U}$ on $N_{x}^{*}$ with embedding $j: N_{x}^{*} \rightarrow Z$, such that $j(W[x]) \unlhd \mathcal{P}$. Let us work now in the universe $L\left[j(T), j\left(N_{x}^{*} \mid \eta_{0}\right)\right]$, where $T$ is the tree of a $\Gamma_{0}$-scale on $U_{0}$. We have $\mathcal{M}$ and the restriction of $\Omega$ to trees in $L\left[j(T), j\left(N_{x}^{*} \mid \eta_{0}\right)\right]$ which are countable in $V$, as this can be computed from $j(T)$. It follows that the tree $\mathcal{T}$ leading from $\mathcal{M}$ to $\mathcal{P}$ is in $L\left[j(T), j\left(N_{x}^{*} \mid \eta_{0}\right)\right]$, and in this universe, it represents $\mathcal{M}$ iterating past $j(W[x])$ without the latter moving. However, $j(W)$ is the output of a maximal $K^{c}$-construction in $L\left[j(T), j\left(N_{x}^{*} \mid \eta_{0}\right)\right]$, and the proof of universality (11.1) applied shows that therefore $\mathcal{M}$ cannot iterate past $j(W)[x]=j(W[x])$ in $L\left[j(T), j\left(N_{x}^{*} \mid \eta_{0}\right)\right]$. This contradiction proves Claim 2.

Again, let $T$ be the tree of a $\Gamma_{0}$-scale on $U_{0}$. In $L\left[T, N_{x}^{*} \mid \eta_{0}\right]$ there are trees $S, S^{*}$ on $\omega \times \eta_{0}$ which project to $A$ and its complement in all size $<\eta_{0}$ generic extensions, and whose images under iterations according to $\Sigma_{x}$ also have this property. (Take $S$ and $S^{*}$ to be trees coming from $\Gamma_{0}$-scales coded into $U_{0}$, and note that $\Sigma_{x}$ moves its tree for $U_{0}$ corrrectly.) By Claim 2, $S, S^{*} \in W[x]$. In $W[x], S$ and $S^{*}$ are $<\eta_{0}$ absolute complements, and hence $p[S]$ is $\operatorname{Hom}_{\eta_{2}}$. Now $W[x]$ has enough Woodin cardinals that its iteration strategy moving $S$ and $S^{*}$ consistently with $A$ guarantees

$$
W[x] \models \text { there are } n+5 \text { Woodin cardinals }<\eta_{2},
$$

and

$$
W[x] \models \exists A \in \operatorname{Hom}_{\eta_{2}}((\mathrm{HC}, \in, A) \models \varphi[y]) .
$$

Using the Woodin cardinals of $W$ between $\eta_{k}$ and $\eta_{2}$ and Woodin's $\left(\Sigma_{1}^{2}\right)^{\text {Hom }_{\infty}}$ generic absoluteness theorem $([25, \S 5])$, we get

$$
W \models \text { there are } n+5 \text { Woodin cardinals }<\eta_{2},
$$

and

$$
W \models \exists A \in \operatorname{Hom}_{\eta_{2}}((\mathrm{HC}, \in, A) \models \varphi[y]) .
$$

Thus $W$ is the desired $\langle\varphi, y\rangle$-witness. This completes the proof of 14.1 .

Combining 9.19 with 14.1, we have a proof of $\boldsymbol{S}_{\beta^{*}+\omega}$ under the smallness assumption $(\dagger)$. That is,

Corollary 14.4 Assume $\mathrm{AD}^{+}$and $(\dagger)$; then for all limit $\lambda, \mathrm{S}_{\lambda}$ holds, and hence MSC holds.

\section{The consistency strength of $\mathrm{AD}^{+}+\theta_{0}<\theta$.}

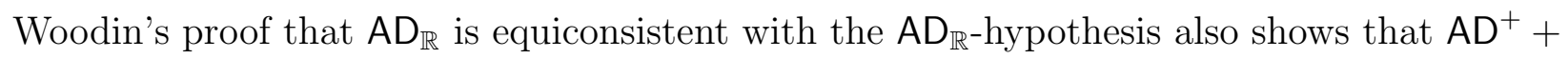
$\theta_{0}<\theta$ is equiconsistent with ZFC plus the existence of one cardinal which is strong to 
the sup of $\omega$ Woodin cardinals. Both results are tightly connected to MSC at the level in question. In this section, we shall give a fairly complete sketch of a different proof of Woodin's equiconsistency result for $\mathrm{AD}^{+}+\theta_{0}<\theta$, one that uses the method by which we have obtained MSC at this level.

Theorem 15.1 (Woodin) The following theories are equiconsistent:

(a) $\mathrm{ZF}+\mathrm{AD}^{+}+\theta_{0}<\theta$,

(b) ZFC $+\exists \kappa \exists \lambda(\lambda$ is a limit of Woodin cardinals, and $\kappa$ is $<\lambda$-strong $)$.

Proof sketch. Given $M$ satisfying the theory in (b), its derived model at $\lambda$ satisfies the theory in (a), by theorem 2.11 .

For the other direction, let us assume $\mathrm{ZF}+\mathrm{AD}^{+}+\theta_{0}<\theta$. We may also assume that we are in the minimal model of $\mathrm{ZF}+\mathrm{AD}^{+}+\theta_{0}<\theta$ containing all reals and ordinals, that is, that there is no $\xi<\theta$ such that $L\left(P_{\xi}\right) \cap P(\mathbb{R})=P_{\xi}$, and $L\left(P_{\xi}\right) \models \theta_{0}<\theta$. Thus by the preceding sections, we have MSC, in fact, we have $S_{\lambda}$ for all limit $\lambda$. We shall construct a class model of the theory in (b).

Fix an sjs $\vec{A}$ which seals $\Sigma_{1}^{2}$, and a real $x_{0}$ such that each $A_{i}$ is $\operatorname{OD}\left(x_{0}\right)$. Woodin's proof that AD yields inner models with $\omega$ Woodin cardinals shows that for each countable transitive set $c$, there is a proper class model $M_{\omega}^{\vec{A}}(c)$ over $c$ with $\omega$ Woodins, with the property that all its countable elementary submodels are $\omega_{1}$-iterable $\vec{A}$-mice. (Hence they are iterable in a way that moves the $\vec{A}$-term relations correctly.)

We need to recall some features of the way we translated certain generic extensions $\mathcal{Q}[g]$ of ordinary ms-mice $\mathcal{Q}$ into $\vec{A}$-mice $\mathcal{Q}[g]^{a}$, replacing extenders with applications of the $\oplus \vec{A}$ operation. (See section 13.) This relied on two hybrid mouse operators $\mathcal{H}_{0}^{\vec{A}}$ and $\mathcal{H}_{1}^{\vec{A}}$, and on being above a real which enables one to compute $\mathcal{H}_{1}(x)$ from $\mathcal{H}_{0}(x)$. In the present context, we have

$$
\mathcal{H}_{0}^{\vec{A}}(b)=b \oplus \vec{A},
$$

for $b$ in the HC-cone above $x_{0}$, as before. The $\mathcal{H}_{1}$ operator of section 12 depended on a coarse mouse operator $x \mapsto\left(M_{x}, N_{x}^{*}, \Sigma_{x}, \delta_{x}\right)$ capturing $\vec{A}$ and perhaps more. We do not have such a coarse mouse operator before us now, and in some sense, we have to consider all possible ones. But now for each coarse mouse operator we could choose, the associated $\mathcal{H}_{1}$ operator will be intercomputable with $\mathcal{H}_{0}$ on an $\mathrm{HC}$ cone via some effective process coded into some real $z$. (This process is described at the end of section 12 , where the real in question is called $z_{0}$.) Let us call a real $z$ a potential code iff

(i) $z$ determines an operator $\mathcal{H}_{1}^{\vec{A}, z}$ defined on the HC cone above $x_{0}, z$, via the procedure for computing $\mathcal{H}_{1}^{\vec{A}}(b)$ from $\mathcal{H}_{0}^{\vec{A}, z}(b)$, and vice-versa, described at the end of section 12 , and 
(ii) the resulting operator $\mathcal{H}_{1}^{\vec{A}, z}$ behaves sufficiently like the $\mathcal{H}_{1}^{\vec{A}}$ operator of section 12 .

We shall leave it to the reader to determine what "sufficiently like" means, from the proof to follow. The main thing is that the set of potential codes is projective in $\vec{A}$, and if $z$ is actually the $z_{0}$ of section 12 associated to some coarse mouse operator capturing $\vec{A}$, then $z$ is a potential code.

Let us call $c$ adequate iff $c$ is a countable transitive structure with the relevant first order properties of one of the background universes $N_{x}^{*} \mid \delta_{x}$ as in section 10 . We leave it to the reader to fully abstract these properties from the argument to follow. Among them are ZFC, that there is a distinguished real $x=x(c)$, and that the $L[\vec{E}, x]$-construction succeeds in producing a model $N$ with a strong cardinal. If $c$ has these properties, we write $N=N^{c}$ and $\kappa=\kappa^{c}$.

Let us call $\langle c, z\rangle$ nice iff $z$ is a potential code, $c$ is adequate, and $x_{0}, z \leq_{T} x(c)$.

Finally, let us call $\langle c, z\rangle$ good iff

(1) $\langle c, z\rangle$ is nice,

(2) $M_{\omega}^{\vec{A}}(c) \models o(c)$ is Woodin, and for all $\alpha<o(c), P(\alpha) \cap M_{\omega}^{\vec{A}}(c) \subseteq c$, and

(3) there is a proper class premouse $\mathcal{Q}$ such that $N^{c} \unlhd \mathcal{Q}, \kappa^{c}$ is the unique cardinal strong past $o(c)$ in $\mathcal{Q}$, and it is strong in $\mathcal{Q}$ to the sup of the Woodin cardinals in $M_{\omega}^{\vec{A}}(c)$, and using $\mathcal{H}_{0}^{\vec{A}}$ to translate away extenders from $\mathcal{Q}$ overlapping $o(c)$, we get

$$
\mathcal{Q}[c]^{a}=M_{\omega}^{\vec{A}}(c)
$$

moreover, starting with $M_{\omega}^{\vec{A}}(c)$ and using $\mathcal{H}_{1}^{\vec{A}, z}$ to invert this translation, we get back $\mathcal{Q}$.

The translation from $\mathcal{Q}[c]$ to $\mathcal{Q}[c]^{a}$ is defined more carefully in section 13 . We shall show that there is a good $c$. It is easy then to see that the associated $\mathcal{Q}$ is a model of the theory in (b). The Woodin cardinals of $M_{\omega}^{\vec{A}}(c)$ are Woodin in $\mathcal{Q}$, and $\kappa^{c}$ is strong in $\mathcal{Q}$ to their sup.

Claim. There is a good $\langle c, z\rangle$.

Proof. Assume not.

For $\langle c, z\rangle \in \mathrm{HC}$, put

$$
F(c, z)=0 \Leftrightarrow\langle c, z\rangle \text { is not nice . }
$$

Suppose now $\langle c, z\rangle$ is nice. The key is that the translation process is step-by-step invertible, so that clauses (2) and (3) can be expressed in the form

$$
M_{\omega}^{\vec{A}}(c) \models \varphi[c, z],
$$


where $\varphi$ is a $\Pi_{1}$ formula in the language of $\vec{A}$-mice. Since one of (2) and (3) fails, we can set

$$
\begin{aligned}
F(c, z)= & \text { minimal } \omega_{1} \text {-iterable } \vec{A} \text {-mouse } \mathcal{P} \text { over } c \\
& \text { such that } \mathcal{P} \models \neg \varphi[c, z] .
\end{aligned}
$$

Remark 15.2 The reader may wonder how one can express with a $\Pi_{1}$ assertion over $M_{\omega}^{\vec{A}}(c)$ that $\kappa^{c}$ is strong to the sup of the Woodins in the structure $\mathcal{Q}$ obtained by inverting the translation procedure. The reason is just that our $\Pi_{1}$ formula $\varphi$ can just say that every level of $M_{\omega}^{\vec{A}}(c)$ that is of the form $P \oplus \vec{A}$ yields an extender with critical point $\kappa^{c}$ through the inversion process given by $\mathcal{H}_{1}^{\vec{A}, z}$. There is a one-one correspondence between $\vec{A}$-active levels of $M_{\omega}^{\vec{A}}(c)$ and extenders with critical point $\kappa^{c}$ which overlap $o(c)$, and occur finitely many ultrapowers, by such overlapping extenders, away from $\mathcal{Q}$.

$F$ is a total function on $\mathrm{HC} \times \mathrm{HC}$, and it is clear by inspection that $F$ is $\Delta_{1}^{2}\left(\vec{A}, x_{0}\right)$. This gives us a good scaled pointclass containing $F$, and thus a way to "beat $F$ " with some c. More precisely, let $f: \mathbb{R} \rightarrow \mathbb{R}$ be such that whenever $u \in \mathbb{R}$ codes a $\langle c, z\rangle$, then $f(u)$ codes $F(c, z)$, and $f$ is $\Delta_{1}^{2}\left(\vec{A}, x_{0}\right)$. Let $x \mapsto\left(M_{x}, N_{x}^{*}, \Sigma_{x}, \delta_{x}\right)$ be a coarse mouse operator that captures $\vec{A}$ and $\{(u, n, m) \mid f(u)(n)=m\}$. From this coarse operator we get an operator $\mathcal{H}_{1}^{\vec{A}}$ and a code $z$ with the properties of $z_{0}$ at the end of section 12 . $z$ is a potential code, and we have $\mathcal{H}_{1}^{\vec{A}}=\mathcal{H}_{1}^{\vec{A}, z}$. Fix $x$ such that $x_{0}, z \leq_{T} x$, and set $c=N_{x}^{*} \mid \delta_{x}$.

Clearly, $\langle c, z\rangle$ is nice. Let $\mathcal{P}=F(c, z)$, so that $\mathcal{P}$ is an $\omega_{1}$-iterable $\vec{A}$-mouse over $c$, and

$$
\mathcal{P} \models \neg \varphi[c, z]
$$

Also, $\mathcal{P} \in N_{x}^{*}$, because our coarse operator captured what it did. Let us now work in $N_{x}^{*}$, where $o(c)=\delta_{x}$ is Woodin, and form a Skolem hull embedding

$$
\pi: S \rightarrow N_{x}^{*} \mid \tau
$$

for some large $\tau$, with $S$ transitive, and $\eta=\operatorname{crit}(\pi)$ an inaccessible cardinal of $N_{x}^{*}$, and

$$
\pi(\eta)=\delta_{x}
$$

and everything relevant in $\operatorname{ran}(\pi)$. In particular, let

$$
\pi(\overline{\mathcal{P}})=\mathcal{P}
$$

We also arrange $\kappa^{c}<\eta$, and that $\eta$ is not Woodin in $N^{c}$, which we can do because otherwise, some proper initial segment of $N^{c}$ already satisfies the theory in (b) of our theorem.

Set

$$
\mathcal{Q}=N^{c} \mid \gamma
$$


where $\gamma>\eta$ is least such that over $N^{c} \mid \gamma$ one can define a function $g: \eta \rightarrow \eta$ witnessing that $\eta$ is not Woodin. Set also $\bar{c}=N_{x}^{*} \mid \eta$, so that $\pi(\bar{c})=c$. Now $\bar{c}$ is generic over $\mathcal{Q}$ for the extender algebra of $\mathcal{Q}$ at $\eta$, and moreover, we can form $\mathcal{Q}[\bar{c}]^{a}$ by the construction of section 13. Both $\mathcal{Q}[\bar{c}]^{a}$ and $\overline{\mathcal{P}}$ are $\omega_{1}$-iterable sound $\vec{A}$-mice over $\bar{c}$ which project to $\bar{c}$, so one is an initial segment of the other. Since $\mathcal{Q}[\bar{c}]^{a}$ can recover $\mathcal{Q}$, it defines a failure of $\eta$ to be Woodin, whereas $\overline{\mathcal{P}}$ defines no such failure. Thus $\overline{\mathcal{P}} \unlhd \mathcal{Q}[\bar{c}]^{a}$. But

$$
\mathcal{Q}[\bar{c}]^{a} \models \varphi[\bar{c}, z]
$$

because $\mathcal{Q}[\bar{c}]^{a}$ was in reality obtained by the translation procedure using $\mathcal{H}_{0}^{\vec{A}}$, and $\mathcal{H}_{1}^{\vec{A}, z}$ to invert it. Since $\varphi$ is $\Pi_{1}$, we have

$$
\overline{\mathcal{P}} \models \varphi[\bar{c}, z] .
$$

This contradicts the elementarity of $\pi$, and proves the claim.

The claim easily yields the theorem, as we described above.

The author believes that this argument can be pushed to as to prove Woodin's result that $\operatorname{Con}\left(A D_{\mathbb{R}}\right) \Rightarrow \operatorname{Con}\left(A D_{\mathbb{R}}\right.$-hypothesis $)$. He has not fully done so, however.

\section{Global MSC implies the local MSC}

In this section, we prove

Theorem 16.1 Assume $\mathrm{AD}^{+}$and $\mathrm{MSC} 2$; then for all $\beta, \mathrm{S}_{\beta}$ holds.

The theorem is of no direct use in proving MSC, but it does suggest that trying to prove $S_{\lambda}$ by induction on $\lambda$ is a reasonable approach. The proof of the theorem may also indicate some features one should look for in an inductive proof of $S_{\beta}$.

We remark on a more general local form of MSC at the end of this section.

Lemma 16.2 Assume $\mathrm{AD}^{+}$and $\mathrm{MSC}$. Let $\Gamma$ be a good scaled pointclass which is not closed under $\exists^{\mathbb{R}}$, with $\Gamma \subseteq \Delta_{1}^{2}$; then there is a mouse operator $\mathcal{M}$ which is coded by a set of reals which is projective in some $\Gamma$ set, such that for all $a \in \operatorname{dom}(\mathcal{M}), C_{\Gamma}(a)=P(a) \cap \mathcal{M}(a)$.

Proof. Let $\Gamma^{*}=\exists^{\mathbb{R}} \forall^{\mathbb{R}} \Gamma$ and for any real $r$ let $H(r)$ denote the universal $\Gamma^{*}(r)$ subset of $\omega$. $H$ is a jump operator in the sense of [28]. By 4.2 (2), we have a mouse jump operator $\mathcal{N}$ such that $\mathcal{N} \equiv_{m} H$. Let $\mathcal{M}$ be the HC-extension of $\mathcal{N}$ (cf. 4.5$)$. It is easy to see that $C_{\Gamma}(a) \subseteq \mathcal{M}(a)$, for an HC-cone of $a$. We can get $P(a) \cap \mathcal{M}(a) \subseteq C_{\Gamma}(a)$ by taking $\mathcal{M}(a)$ minimal. 
It is worth noting that nothing in Lemma 16.2 requires that the unique $\omega_{1}$ strategies for the $\mathcal{M}(a)$ be found anywhere near $\Gamma$. Indeed, if $\Gamma$ is properly within some $\Sigma_{1}^{2}$ gap, this will not be the case. Nonetheless, the $\mathcal{M}$ operator is itself near $\Gamma$. In the case $\Gamma$ occurs just after a new $\Sigma_{1}^{2}$ fact, we will parlay $\mathcal{M}$ into a mouse witnessing the fact with a strategy near $\Gamma$.

The following little lemma will be useful at one point.

Lemma 16.3 Assume $\mathrm{AD}^{+}$, and let $\mathcal{M}$ be a countable a-premouse which is $\omega_{1}$-iterable (i.e., iterable for normal trees); then $\mathcal{M}$ is $\left(\omega_{1}, \omega_{1}\right)$-iterable (i.e., iterable for stacks of normal trees).

Proof. Let $\Sigma$ be an $\omega_{1}$-iteration strategy for $\mathcal{M}$, which by the Basis theorem we may assume is Suslin and co-Suslin. Let $x \mapsto\left(M_{x}, \Sigma_{x}, N_{x}^{*}, \delta_{x}\right)$ be a coarse mouse operator capturing (a set of reals coding) $\Sigma$. Let $x$ be in the domain of this operator, with $\mathcal{M}$ coded by a real recursive in $x$. Let $N$ be the output of the maximal full background extender construction of $N_{x}^{*}$, done over $a . N_{x}^{*}$ knows how to iterate $\mathcal{M}$, and the proof of universality from 11.1 shows that $\mathcal{M}$ embeds into an $\Omega$-iterate of $N$, where $\Omega$ is the strategy for $N$ induced by $\Sigma_{x}$. (A little more work would show $\mathcal{M}$ embeds into some initial segment of $N$ itself.) But $\Omega$ is an $\left(\omega_{1}, \omega_{1}\right)$-strategy, so by pulling back, we have such a strategy for $\mathcal{M}$.

Proof of Theorem 16.1. Assume $\mathrm{AD}^{+}$and MSC2. We shall prove $\mathrm{S}_{\lambda}$ for limit $\lambda$, and leave the minor adjustment needed for the full proof to a remark. Suppose $A, y$, and $\Gamma^{-}$are such that

$$
(\mathrm{HC}, \in, A) \models \varphi[y]
$$

with $y \in \mathbb{R}$ and $A \in \Gamma^{-}$, and $\Gamma^{-}$a good scaled pointclass not closed under $\forall^{\mathbb{R}}$. Suppose $\varphi$ is a $\Sigma_{k}$ formula, and let $\Gamma$ be, in the inclusion order, the $k+5$-th good scaled pointclass containing $\Gamma^{-}$. It will be enough to find a $\langle\varphi, y\rangle$-witness $\mathcal{P}$ such that $\mathcal{P}$ has an $\omega_{1}$-iteration strategy which is in $L_{2}(\Gamma, \mathbb{R})$.

Let $\mathcal{M}$ and $r_{0}$ be as in Lemma 16.2 with respect to $\Gamma$. We may assume $y \leq_{T} r_{0}$.

Claim 2. There is a function $J$ defined on all countable, transitive, self-wellordered $a$ such that $r_{0} \in a$, and such that for each such $a$ :

(a) $J(a)$ is a sound, $\omega_{1}$-iterable mouse over $a$, and

(b) either $\mathbb{R} \cap a \neq \mathbb{R} \cap J(a)$, or $J(a) \models \mathrm{ZFC}^{-}+|a|^{+}$exists, and

$$
\begin{aligned}
J(a) \models & \text { there are trees } T, U \text { on } \omega \times \omega_{1} \text { which are absolute complements } \\
& \text { for } \operatorname{Col}(\omega, a) \text { and such that }(\mathrm{HC}, \in, p[T]) \models \varphi[y] .
\end{aligned}
$$

and 
(c) $J(a)$ is the least $a$-mouse satisfying (a) and (b).

Moreover, $J$ is coded by a set of reals which is in $L_{2}(\Gamma, \mathbb{R})$.

Proof. It is enough to define, over $L_{1}(\Gamma, \mathbb{R})$, a mouse $J_{0}(a)$ satisfying (a) and (b). We can then just let $J(a)$ be the first initial segment of $J_{0}(a)$ satisfying (a) and (b).

We define $J_{0}(a)$ by closing under the $\mathcal{M}$-operator. More precisely, set

$$
\mathcal{P}_{0}=\mathcal{M}(a) .
$$

Suppose now we have $\mathcal{P}_{\xi}$. If $\mathbb{R} \cap \mathcal{M}\left(\mathcal{P}_{\xi}\right) \nsubseteq a$, then we stop our induction, and let $J_{0}(a)$ be the first level of $\mathcal{M}\left(\mathcal{P}_{\xi}\right)$ which contains a real not in $a$. $\left(J_{0}(a)\right.$ is the first such level re-arranged as an $a$-mouse; it will follow from the construction that this is possible.) If $\mathbb{R} \cap \mathcal{M}\left(\mathcal{P}_{\xi}\right) \subseteq a$, we set

$$
P_{\xi+1}=\mathcal{M}\left(P_{\xi}\right) .
$$

If $\mathcal{P}_{\xi+1} \models \omega_{1}$ exists, then we stop the induction. Finally, for $\lambda$ a countable limit ordinal,

$$
\mathcal{P}_{\lambda}=\bigcup_{\alpha<\lambda} \mathcal{P}_{\alpha} .
$$

It is easy to see that $\mathcal{P}_{\eta}$ is a sound mouse over $a$ whenever it is defined, and $\mathcal{P}_{\eta}$ projects to $a$ at all stages $\eta$ except possibly the last, when we may have $\eta=\xi+1$ and $\rho_{\omega}\left(\mathcal{P}_{\eta}\right)=\omega_{1}^{\mathcal{P}_{\eta}}=o\left(\mathcal{P}_{\xi}\right) .{ }^{10}$

Since there is no $\omega_{1}$-sequence of distinct subsets of $a$, our induction must stop at some countable stage. If it stops because a new real has been constructed, then we have already defined $J_{0}(a)$. If not, suppose it stops with $\mathcal{P}_{\xi+1}=\mathcal{M}\left(\mathcal{P}_{\xi}\right)$ for the second reason. Let $T_{\Gamma}$ be the tree of a $\Gamma$-scale on a universal $\Gamma$ set. Note that $o\left(\mathcal{P}_{\xi}\right)$ is the $\omega_{1}$ of $L\left[T_{\Gamma}, \mathcal{P}_{\xi}\right]$, and in $L\left[T_{\Gamma}, \mathcal{P}_{\xi}\right]$ we have a pair $\left(T_{0}, U_{0}\right)$ of trees which project to $A$ and $\mathbb{R} \backslash A$ in $V$. Working in $L\left[T_{\Gamma}, \mathcal{P}_{\xi}\right]$ and taking a Skolem hull, we get trees $(T, U)$ on $\omega \times o\left(\mathcal{P}_{\xi}\right)$ which project in any $\operatorname{Col}(\omega, a)$-generic extension of $L\left[T_{\Gamma}, \mathcal{P}_{\xi}\right]$ to the intersections of $A$ and $\mathbb{R} \backslash A$ with that extension. But then $(T, U) \in \mathcal{P}_{\xi+1}$, so we can set $J_{0}(a)=\mathcal{P}_{\xi+1} \mid \nu$ (re-arranged as mouse over $a$ ), where $T, U \in \mathcal{P}_{\xi+1} \mid \nu$ and $\mathcal{P}_{\xi+1}|\nu|=\mathrm{ZFC}^{-}$. It is clear that $J_{0}(a)$ thinks $T$ and $U$ are $\operatorname{Col}(\omega, a)$-absolutely complementing, so to show that (b) holds, it is enough to see that $(\mathrm{HC}, \in, p[T])^{J_{0}(a)} \prec_{\Sigma_{k}}(\mathrm{HC}, \in, A)$. But $p[T]^{J_{0}(a)}=A \cap a$ by construction, and the $\Sigma_{k^{-}}$ elementarity holds because $\mathbb{R} \cap a=\mathbb{R} \cap J_{0}(a)$ is $C_{\Gamma}$-closed, and we took $\Gamma$ to be $k$ good scaled pointclasses above $\Gamma_{0}$, where $A \in \Gamma_{0}$.

This proves claim 2 .

Fix $J$ as in claim 2. $J(a)$ is the unique $a$-mouse satisfying a certain first order theory, which we call $T^{J}$. (This is a theory in the language of set theory, expanded by a name for a.) Therefore, we have condensation:

\footnotetext{
${ }^{10}$ We do not have any form of condensation for the $\mathcal{M}$-operator, and this blocks the obvious attempt to prove $\mathcal{P}_{\eta}$ projects to $a$ in this case.
} 
Claim 3. I $\pi: P \rightarrow J(a)$ is fully elementary, and $r_{0}$ is in the range of $\pi$ then $P=J\left(\pi^{-1}(a)\right)$.

Definition 16.4 Let a be self-wellordered, with $r_{0} \in a$.

(a) An a-premouse $\mathcal{N}$ is $J$-closed iff $J(\mathcal{N} \mid \xi)$ is an initial segment of $\mathcal{N}$, for every cardinal $\xi$ of $\mathcal{N}$.

(b) $\mathcal{N}$ is $T^{J}$-closed iff for every cardinal $\xi$ of $\mathcal{N}$, there is a $\gamma \leq o(\mathcal{N})$ such that $(\mathcal{N}|\gamma, \mathcal{N}| \xi) \mid=$ $T^{J}$. We write $J^{\mathcal{N}}(\mathcal{N} \mid \xi)=\mathcal{N} \mid \gamma$ in this case.

(c) $\mathcal{N}$ is $J$-correct iff whenever $J^{\mathcal{N}}(\mathcal{N} \mid \xi)=\mathcal{N} \mid \gamma$, then $J(\mathcal{N} \mid \xi)=\mathcal{N} \mid \gamma$.

(d) By $M_{n}^{J}(a)$ we mean the least $\omega_{1}$-iterable a-mouse $\mathcal{M}$ such that some ordinals $\delta_{0}<\ldots<$ $\delta_{n-1}$ are Woodin cardinals of $\mathcal{M}, \mathcal{M} \mid \delta_{n-1}$ is $J$-closed, and $\mathcal{M}=J\left(\mathcal{M} \mid \delta_{n-1}\right)$.

Clearly, an $\omega_{1}$-iterable model is $J$-correct, and hence $T^{J}$-closed iff it is $J$-closed. So $M_{n}^{J}(a)$ is just the least iterable $a$-mouse satisfying a certain theory.

Claim 4. $M_{n}^{J}(a)$ exists, for every $n$ and every selfwellordered $a$ with $r_{0} \in a$. Moreover, fixing $n$, the operator $a \mapsto M_{n}^{J}(a)$ is coded by a set of reals which is in $L_{2}(\Gamma, \mathbb{R})$.

Proof. Let $\Gamma_{0}$ be a good scaled pointclass containing $\Gamma$, contained in projective-in- $\Gamma$, and such that $J$ is coded by a set of reals in $\Gamma_{0}$. Let $\Gamma_{i}$ be the $i$-th good scaled pointclass including $\Gamma_{0}$. Fix $n \geq 0$, and let $U$ be a universal $\Gamma_{n+5}$ set. By 10.3, we can fix a coarse mouse operator $x \mapsto\left(M_{x}, \Sigma_{x}, N_{x}^{*}, \delta_{x}\right)$ which captures $U$, and which is in $L_{2}(\Gamma, \mathbb{R})$. It is enough to define the desired $a \mapsto M_{n}^{J}(a)$ in a projective way from this operator.

So fix a selfwellordered $a$ with $r_{0} \in a$. Let $x_{0}$ be a real coding $a$, and let $x \geq_{T} x_{0}$ be in the domain of our coarse mouse operator capturing $U$. Working in $N_{x}^{*}$, we shall do a variant of the full background extender construction over $a$. We shall show that all models in this variant construction are $\omega_{1}$-iterable in $V$, and that the construction reaches a level which believes it is $M_{n}^{J}(a)$. By iterability, this level must really be $M_{n}^{J}(a)$. Since the variant construction is uniformly definable over $N_{x}^{*}$ from $a$, we then have that $a \mapsto M_{n}^{J}(a)$ is projective in $x \mapsto\left(M_{x}, \Sigma_{x}, N_{x}^{*}, \delta_{x}\right)$, as desired.

There is no reason as yet to believe the usual construction reaches $M_{n}^{J}(a)$, for although it produces a model $N$ which is universal in $N_{x}^{*}$, we cannot show $J(N \mid \xi) \unlhd N$ without being able to iterate $J(N \mid \xi)$ above $\xi$ inside $N_{x}^{*}$. We know that $J(N \mid \xi)$ is iterable above $\xi$ in $V$, but we have no useful bound on the complexity of its iteration strategy. So we modify the usual construction by simply adding steps which close under $J$. Note here that because our coarse mouse operator captured $U, J \uparrow\left(N_{x}^{*} \mid \delta_{x}\right) \in N_{x}^{*}$.

More precisely, working in $N_{x}^{*}$, we construct an extender model over $a$ via approximations $\mathcal{N}_{\xi}$, for $\xi<\delta_{x}$. If $\mathcal{N}_{\xi}=\left(J_{\alpha}^{\vec{E}}, \in, \vec{E}, \emptyset\right)$ and there is an extender $F$ such that $\left(J_{\alpha}^{\vec{E}}, \in, \vec{E}, F\right)$ is 
a premouse and $F$ has a full background extender, then we pick such an $F$ subject to the usual conditions, and set $\mathcal{N}_{\xi+1}=\left(J_{\alpha}^{\vec{E}}, \in . \vec{E}, F\right)$. If there is no such $F$, or if $\mathcal{N}_{\xi}$ is already active, then we let $\mathcal{Q}$ be the first initial segment of $J\left(\mathcal{N}_{\xi}\right)$ such that $\rho_{\omega}(\mathcal{Q})<o\left(\mathcal{N}_{\xi}\right)$ and $\mathcal{Q}$ is not $\omega$-sound, and put $\mathcal{N}_{\xi+1}=\mathcal{C}_{\omega}(\mathcal{Q})=$ the $\omega$-th core of $\mathcal{Q}{ }^{11}$ If there is no such initial segment $\mathcal{Q}$ of $J\left(\mathcal{N}_{\xi}\right)$, then we just set $\mathcal{N}_{\xi+1}=J\left(\mathcal{N}_{\xi}\right)$. If $\lambda \leq \delta_{x}$ is a limit, then $\mathcal{N}_{\lambda}$ is the lim inf of the $\mathcal{N}_{\alpha}$, for $\alpha<\lambda$, as usual. Finally, we set $N=\mathcal{N}_{\delta_{x}}$.

\section{Subclaim A.}

1. If $\mathcal{N}$ is $J$-correct and $\pi: \mathcal{M} \rightarrow \mathcal{N}$ is elementary, then $\mathcal{M}$ is $J$-correct.

2. Every $\mathcal{N}_{\xi}$ is $J$-correct.

3. Let $\kappa$ be a cutpoint of $\mathcal{N}_{\xi}$ and suppose $\rho_{\omega}\left(\mathcal{N}_{\xi} \mid \gamma\right) \leq \kappa$, and $J\left(\mathcal{N}_{\xi} \mid \gamma\right)$ is not a proper initial segment of $\mathcal{N}_{\xi} \mid \gamma$; then $\mathcal{N}_{\xi} \mid \gamma$ is $\omega_{1}$-iterable above $\kappa$.

Proof. Part (1) follows at once from condensation for $J$. Part (2) is an easy induction on $\xi$, using part (1) to show that coring down preserves $J$-correctness.

Part (3) is a refinement of (2). Suppose $\mathcal{T}$ is a normal iteration tree on $\mathcal{N}_{\xi} \mid \gamma$ above $\kappa$. Let $E$ be the first extender used in $\mathcal{T}$, let $\eta \leq \gamma$ be least such that $\operatorname{lh}(E) \leq \eta$ and $\rho_{\omega}\left(\mathcal{N}_{\xi} \mid \eta\right)=\kappa$, and let $\nu$ be the $\kappa^{+}$of $\mathcal{N}_{\xi} \mid \eta$. Then $\mathcal{T}$ can be regarded as a tree on $\mathcal{N}_{\xi} \mid \eta$. Tracing the ancestry of $\mathcal{N}_{\xi} \mid \eta$ is our construction yields a $\sigma \leq \xi$ and an elementary $\pi: \mathcal{N}_{\xi} \mid \eta \rightarrow \mathcal{N}_{\sigma}$ such that, letting $\mu=\pi(\nu)$, we have that $\mathcal{N}_{\sigma} \unlhd J\left(\mathcal{N}_{\sigma} \mid \mu\right)$. But then $\mathcal{N}_{\sigma}$ has an iteration strategy $\Sigma$ above $\mu$, and we can assume $\mathcal{T}$ is being played by the pullback $\Sigma^{\pi}$, and use $\Sigma^{\pi}$ to continue iterating.

\section{Subclaim B.}

1. If $\kappa$ is a cardinal of $\mathcal{N}_{\xi}$ and $\kappa>$ order-type $(<)$, where $<$ is a $\operatorname{rud}(a)$ wellorder of $a$, then $\mathcal{N}_{\xi} \mid \kappa$ is $J$-closed.

2. An extender $F$ on the sequence of $\mathcal{N}_{\xi}$ has a background extender provided by the construction (perhaps via some resurrection of $F$ ) if and only if $\mathcal{N}_{\xi} \mid \operatorname{lh}(F)$ is $J$-closed.

Proof. Straightforward.

Subclaim C. Each $\mathcal{N}_{\xi}$ is $\left(\omega, \omega_{1}, \omega_{1}\right)$-iterable.

Proof sketch. By Lemma 16.3, it suffices to consider normal iterations. We lift such iterations of $\mathcal{N}_{\xi}$ to iterations of $N_{x}^{*}$ by resurrecting background extenders as in [12], when these exist.

\footnotetext{
${ }^{11} \mathcal{Q}$ is $\omega$-sound when regarded as a mouse over $\mathcal{N}_{\xi}$, but here we are regarding it as a mouse over $a . \mathcal{Q}=\mathcal{N}_{\xi}$ is possible.
} 
The problem here is that we may use an extender which is justified by an application of $J$, and hence has no background extender to be resurrected. Let us consider the first stage at which this happens.

We must be in the following situation. We have $\mathcal{T}$ on $\mathcal{N}_{\xi}$ played according to the strategy of lifting to $N_{x}^{*}$ and using $\Sigma_{x}$ to form a tree $\mathcal{U}$ there. $\mathcal{T}$ has a last model $\mathcal{P}$, and $\mathcal{U}$ has a last model $R$, with

$$
i^{\mathcal{U}}: N_{x}^{*} \rightarrow R
$$

the canonical embedding. There is an embedding

$$
\pi: \mathcal{P} \rightarrow \mathcal{Q},
$$

where $\mathcal{Q}$ is a model on $i^{\mathcal{U}}\left(\left\langle\mathcal{N}_{\gamma} \mid \gamma<\delta_{x}\right\rangle\right)$. We have that all models on $i^{\mathcal{U}}\left(\left\langle\mathcal{N}_{\gamma} \mid \gamma<\delta_{x}\right\rangle\right)$ are $J$-correct, because iterations by $\Sigma_{x}$ move the absolutely complementing trees for $U$ in $N_{x}^{*}$ correctly. So $\mathcal{Q}$, and hence $\mathcal{P}$, are $J$-correct. We are about to use an extender $F$ from the sequence of $\mathcal{P}$ to extend $\mathcal{T}$. Since we cannot resurrect a background extender for $\pi(F)$, $\mathcal{Q} \mid \operatorname{lh}(\pi(F))$ is not $J$-closed, and thus $\mathcal{P} \mid \operatorname{lh}(F)$ is not $J$-closed. Let $\lambda<\operatorname{lh}(F)$ be largest such that

$$
\alpha<\lambda \Rightarrow J(\mathcal{P} \mid \alpha) \unlhd \mathcal{P} \mid \lambda .
$$

This implies that $\lambda<\operatorname{crit}(F)$, and that $\lambda$ is a cutpoint of $\mathcal{P}$ and not the critical point of an extender on the $\mathcal{P}$-sequence. Since $F$ was the first $E_{\alpha}^{\mathcal{T}}$ such that $\mathcal{M}_{\alpha}^{\mathcal{T}} \mid \operatorname{lh}\left(E_{\alpha}^{\mathcal{T}}\right)$ is not $J$-closed, all extenders used in $\mathcal{T}$ before $F$ have length $<\lambda$.

Let $\eta$ be least such that $\operatorname{lh}(F) \leq \eta$ and $\rho_{\omega}(\mathcal{P} \mid \eta) \leq \lambda$. It follows that the rest of our normal $\mathcal{T}$ will have to be a tree on $\mathcal{P} \mid \eta$ which is above $\lambda$. There will be no going back to earlier models of $\mathcal{T}$; instead, we have permanently dropped. So it is enough to see that $\mathcal{P} \mid \eta$ is $\omega_{1}$-iterable above $\lambda$. For that, it is enough that $\mathcal{Q} \mid \pi(\eta)$ is $\omega_{1}$-iterable above $\pi(\lambda)$. But this follows at once from the proof of part (3) of Subclaim A, applied to the models of $i^{\mathcal{U}}\left(\left\langle\mathcal{N}_{\gamma} \mid \gamma<\delta_{x}\right\rangle\right)$. The proof does apply because $i^{\mathcal{U}}$ moves the $J$-operator correctly.

By Subclaim C, the $\mathcal{N}_{\xi}$ are all $\omega$-solid, so that the construction does not stop, and does indeed produce a model $N=\mathcal{N}_{\delta_{x}}$.

Working in $N_{x}^{*}$, let us call $\eta$ a $\Gamma_{i}$-Woodin cardinal iff by using the appropriate absolutely complementing trees to compute $C_{\Gamma}\left(N_{x}^{*} \mid \eta\right)$, we see that $\eta$ is Woodin with respect to all functions in $C_{\Gamma}\left(N_{x}^{*} \mid \eta\right)$. Let us put

$$
\eta_{n}=\text { the least } \Gamma_{n} \text {-Woodin cardinal, }
$$

and for $1 \leq i \leq n$,

$$
\eta_{i-1}=\text { the least } \Gamma_{i-1} \text {-Woodin cardinal }>\eta_{i} .
$$

So $\eta_{n}<\eta_{n-1}<\ldots<\eta_{0}$, and $\eta_{i}$ is $\Gamma_{i}$-Woodin. One can show that $\eta_{n}, \ldots, \eta_{1}$ are Woodin in $\mathcal{N}_{\eta_{0}}$, by an argument very similar to the proof of claim 1 of 14.1. But also, $J\left(\mathcal{N}_{\eta_{0}} \mid \eta_{1}\right) \unlhd \mathcal{N}_{\eta_{0}}$. Thus our construction has reached $M_{n}^{J}(a)$, and the proof of Claim 4 is complete. 
Recall that $\varphi$ was $\Sigma_{k}$. Let us put

$$
R(a)=M_{k+5}^{J}(a),
$$

for $a$ in $\operatorname{dom}\left(M_{k+5}^{J}\right)$. Let $\mathcal{S}$ be the minimal $\langle\varphi, y\rangle$-witness, which exists by MSC. Since $\mathcal{S}$ projects to $\omega$, it has a unique $\omega_{1}$-strategy $\Sigma$. It is enough to show that $\Sigma$ is projective in $\Gamma$. This we shall do by using the $R$-operator to compute $\Sigma$.

Let $x \mapsto\left(M_{x}, \Sigma_{x}, N_{x}^{*}, \delta_{x}\right)$ be a coarse mouse operator in $L_{3}(\Gamma, \mathbb{R})$ which captures a set of reals coding $a \mapsto R(a)$. Let $x$ be in the domain of this operator, with $r_{0}$ and some real coding $\mathcal{S}$ recursive in $x$, and $R$ defined on the HC-cone above $x$. Working in $N_{x}^{*}$, let $N$ be the output of a maximal full background extender construction over $y$ of height $\delta_{x}$. Since $N$ has an $\omega_{1}$ iteration strategy which is $L_{3}(\Gamma, \mathbb{R})$, it will be enough to show that $\mathcal{S} \unlhd N$. So suppose not.

Let us compare $\mathcal{S}$ with $N$. On the $\mathcal{S}$ side we use $\Sigma$ to pick branches, and we fold in a genericity iteration which guarantees that if $\mathcal{T}$ is a tree of limit length on $\mathcal{S}$ which our process has produced, then

$$
\langle\mathcal{T}, x\rangle \text { is } \mathcal{B} \text {-generic over } \mathcal{M}(\mathcal{T}),
$$

where $\mathcal{B}$ is the $\delta(\mathcal{T})$-generator version of the extender algebra determined by the extender sequence of $\mathcal{M}(\mathcal{T})$. It is not a problem to fold such steps into the usual coiteration process, as both $\mathcal{T}$ and $\mathcal{M}(\mathcal{T})$ are being revealed initial-segment-wise as we proceed toward $\operatorname{lh}(\mathcal{T})$. On the $N$ side, we do the iteration at the level of the background universe $N_{x}^{*}$, as in 12.6 and [17].

This process must terminate, and when it does we must have

$$
i: N_{x}^{*} \rightarrow N^{* *},
$$

and

$\mathcal{T}$ on $\mathcal{S}$ according to $\Sigma$, with last model $\mathcal{P}$,

so that

$$
i(N) \unlhd \mathcal{P} .
$$

Now by the proof of 11.1, $i(N)$ is universal in $N^{* *}$, in the sense that no $y$-premouse iterates past it. So we will have the desired contradiction when we show $\mathcal{T} \in N^{* *}$.

The $R$ operator is moved correctly by $i$, so $R \nmid\left(N^{* *} \mid i\left(\delta_{x}\right)+1\right) \in N^{* *}$. We use this to recover $\mathcal{T}$. Suppose that $\lambda \leq \delta_{x}$, and we have already recovered $\mathcal{T} \mid \lambda$ in $N^{* *}$. Let $\delta=\delta(\mathcal{T}\lceil\lambda)$, let $\mathcal{B}$ be the $\delta$-generator extender algebra of $\mathcal{M}(\mathcal{T} \uparrow \lambda)$, and let $g$ be the $\mathcal{B}$ generic object determined by $\langle\mathcal{T}\lceil\lambda, x\rangle$. Let $a$ be selfwellordered and constructibly equivalent to $\left\langle\mathcal{M}(\mathcal{T}\lceil\lambda), g\rangle\right.$. Working in $N^{* *}$, we can find these objects, and then find $R(a)$. 
Let $b=\Sigma(\mathcal{T}\lceil\lambda)$, and $\mathcal{Q}=\mathcal{Q}(b, \mathcal{T}\lceil\lambda)$, which exists because $\mathcal{S}$ projects to $\omega$. As $\delta$ remains Woodin in $\mathcal{Q}, g$ is $\mathcal{B}$-generic over $\mathcal{Q}$. But $g$ codes $\mathcal{T} \uparrow \lambda$, so the $*$-transform $\mathcal{Q}[g]^{*}$ exists. It can be regarded as a mouse over $a$. So regarding it, we have

Claim 5. $\mathcal{Q}[g]^{*} \unlhd R(a)$.

Proof. If not, then we have a proper initial segment $\mathcal{P}$ of $\mathcal{Q}$ such that $\mathcal{P}[g]^{*}=R(a)$. Let $\delta_{i}$ be the $i$-th Woodin cardinal of $R(a)$, and $\gamma=\delta_{k+5}$. As $\gamma$ is a cardinal in $\mathcal{P}[g], \mathcal{P} \mid \gamma[g]$ has the same universe as $R(a) \mid \gamma$. Let $\mathcal{N}=\mathcal{P} \mid \gamma$. It follows that

$$
\mathcal{N}[g] \models \delta_{1}, \ldots, \delta_{k+4} \text { are Woodin, }
$$

and

$$
\mathcal{N}[g] \models \exists A \in \operatorname{Hom}_{\delta_{k+3}}((\mathrm{HC}, \in, A \models \varphi[y]) .
$$

But then a standard absoluteness argument (due to Woodin) using stationary tower forcing shows that the two displayed statements are true in $\mathcal{N}$, not just in $\mathcal{N}[g]$. But this implies that $\mathcal{N}$ is a $\langle\varphi, y\rangle$-witness. Since $\mathcal{S}$ was the minimal such witness, and we have dropped in going from $\mathcal{S}$ to $\mathcal{N}$, this is a contradiction.

By claim 5, we can recover $\mathcal{Q}$ from $R(a)$ by inverting the $*$-transform. But $\mathcal{Q}$ determines $b$, and hence we can recover $b$ as well. This shows $\mathcal{T} \in N^{* *}$, the desired contradiction. We conclude that in fact $\mathcal{S} \unlhd N$, and hence $\mathcal{S}$ has an $\omega_{1}$ iteration strategy which is in $L_{3}(\Gamma, \mathbb{R})$. This completes the proof of $S_{\lambda}$ for limit $\lambda$.

One gets the full Theorem 16.1 by using 10.2 in the places where we used 10.3. This enables one to remain within the projective-in- $\Gamma$ sets. In the end, our iteration strategy for $\mathcal{S}$ is projective in $\Gamma$, as required.

Remark 16.5 Even our local MSC is not as local as one might wish, because it says nothing about how sets of reals properly inside $\Sigma_{1}^{2}$ gaps are captured locally by mice. We believe that the proof of theorem 16.1 can be modified so as to show

Theorem 16.6 Assume $\mathrm{AD}^{+}+\mathrm{MSC}$, and let $A \subseteq \mathbb{R}$ be Suslin and co-Suslin. Then there is an ms-premouse $\mathcal{M}$, and some $g$ generic over $\mathcal{M}$ for some $\operatorname{Col}(\omega, \kappa)$, and a Woodin cardinal $\delta>\kappa$ of $\mathcal{M}$, and an $\omega_{1}$ iteration strategy $\Sigma$ for $\mathcal{M}$ in the window $(\kappa, \delta)$ such that $(\mathcal{M}[g], \Sigma)$ captures $A$.

The conclusion means that there are $\delta$-absolutely complementing trees $T, U \in \mathcal{M}[g]$ such that for all reals $x, x \in A$ iff there is a $\Sigma$-iteration map $i$ such that $x \in p[i(T)]$. Thus theorem 16.6 is a fine-structural refinement of theorem 10.2; the capturing mouse is a generic extension of an ms-premouse now. One needs the generic extension.

Another localization question one can ask is: suppose $(\mathcal{M}, \lambda)$ is tractable, and let $\Gamma$ be a Wadge initial segment of $\operatorname{Hom}_{I}^{*}$, for some $\mathbb{R}$-genericity iteration of $\mathcal{M}$. Must $L(\Gamma, \mathbb{R})$ be the derived model of some ms-mouse? We do not know the answer. 


\section{MSC implies capturing via $\mathbb{R}$-mice}

In this section, we prove

Theorem 17.1 Assume $\mathrm{AD}^{+}+\mathrm{MSC}$. Let $\alpha$ begin a $\Sigma_{1}^{2}$ gap, and suppose $\Gamma=\left(\Sigma_{1}^{2}\right)^{P_{\alpha}}$ is closed under real quantification. Let $U$ be a universal $\Gamma$ set, and let $A \in \operatorname{Env}(\Gamma)$; then there is an ms-mouse $M$ over $\mathbb{R}$ such that

(a) $M$ is $\mathbb{R}$-sound, and $A$ is definable over $M$, but not in $M$,

(b) for each $k<\omega$, the $\Sigma_{k}$-theory in $M$ with parameters from $\mathbb{R} \cup p_{k}(M)$ is projective in $\langle A, U\rangle$, and

(c) every countable elementary submodel of $M$ has an $\omega_{1}$ iteration strategy coded by a set of reals in $P_{\alpha}$.

Proof. Assume $\Gamma, U, A$ are a counterexample. By Woodin's basis theorem, we may assume $A, U$ are $\boldsymbol{\Delta}_{\mathbf{1}}^{\mathbf{2}}$. We also assume that $A$ is a Wadge-minimal counterexample with respect to $\Gamma$. Letting

$$
\mathcal{B}=\left\{B \mid B<_{w} A\right\}
$$

and

$$
S=\text { minimal } \mathbb{R} \text {-mouse } P \text { such that } \mathcal{B} \subseteq P,
$$

we have then that every countable elementary submodel of $S$ has an $\omega_{1}$-iteration strategy which is in $P_{\alpha}$. Let $S_{0}$ be a set of reals which is projective in $\langle A, U\rangle$, and codes $S$ in some natural way. It is easy to see there is such a set.

Claim 1. $L[S] \cap P(\mathbb{R})=\mathcal{B}$.

Proof. Otherwise, the first level of $L[S]$ past $S$ itself which projects to $\mathbb{R}$ would be an $\mathbb{R}$ mouse $M$ as required in the theorem. We show this now. Part (c) is clear. Let $k$ be least such that $\rho_{k}(M)=\mathbb{R}$, and let $C$ be the $\Sigma_{k}$ theory of $M$ with parameters from $\mathbb{R} \cup p_{k}(M)$. Wadge's lemma gives $A$ is Wadge reducible to either $C$ or $\mathbb{R} \backslash C$, so part (a) holds. For (b), note that $C$ is a wellordered union of sets of reals in $\mathcal{B}$. Moreover, there is a pointclass $\Omega$ contained in the projective-in- $A$ sets such that $A \in \Omega$, and $\Omega$ is closed under wellordered unions. It follows that $C$ is projective in $A$, and from that we easily get (b).

Now let $x_{0} \in \mathbb{R}$ be such that whenever $\sigma$ is a countable set of reals with $x_{0} \in \sigma$, then $A \cap \sigma \in C_{\Gamma}(\sigma)$. Let $\Omega$ be an inductive-like pointclass with the scale property containing $A, U$, and their complements, and let $T$ be the tree of an $\Omega$-scale on a universal $\Omega$ set. Put

$$
N=L\left[T, x_{0}\right]
$$


and

$$
A^{*}=A \cap N, S_{0}^{*}=S_{0} \cap N, \text { and } U^{*}=U \cap N .
$$

Using the Skolem functions given by $T$, we get

$$
\left(\mathrm{HC}^{N}, \in, A^{*}, U^{*}\right) \prec(\mathrm{HC}, \in, A, U) .
$$

Now it is part of the first order theory of $(\mathrm{HC}, \in, A, U)$ that there is no $M$ satisfying (a)-(c) of the theorem. (Note $P_{\alpha}=\left\{B \mid B<_{w} U\right\}$.) It is also part of this theory that $A$ is Wadge minimal having this property with respect to $U$. It follows that

$$
\begin{aligned}
N \models & \neg \exists M(M \text { is sound, projects to } \mathbb{R} \text {, and } \\
& A^{*} \text { is definable over } M \text {, and the theory } \\
& \text { of } M \text { with real parameters is projective in }\left\langle A^{*}, U^{*}\right\rangle, \text { and } \\
& \text { every countable elementary submodel of } M \text { has an } \\
& \left.\omega_{1} \text { iteration strategy coded by a set of reals }<_{w} U^{*}\right) .
\end{aligned}
$$

But $x_{0} \in \mathbb{R}^{N}$, so $A^{*} \in C_{\Gamma}\left(\mathbb{R}^{N}\right)$, and thus by MSC, we can fix a mouse $Q$ over $\mathbb{R}^{N}$ such that $A^{*} \in Q$, and $Q$ has an $\omega_{1}$ iteration strategy $\Sigma$ in $\Gamma$. Since $N$ has $T$ in it, we get that $Q \in N$, and $\Sigma^{*} \in Q$, where $\Sigma^{*}$ is the restriction of $\Sigma$ to trees in $N$ of size $<\omega_{1}^{V}$. (Note $\omega_{1}^{V}$ is measurable in $N$.) It follows that

$$
\begin{aligned}
N \models & \text { every countable elementary submodel of } Q \text { has an } \\
& \omega_{1} \text { iteration strategy coded by a set of reals }<_{w} U^{*} .
\end{aligned}
$$

Working in $N$, let $\mathcal{B}^{*}=\left\{B \subseteq \mathbb{R} \mid B<_{w} A^{*}\right\}$, and let $S^{*}$ be the mouse over $\mathbb{R}^{N}$ coded by $S_{0}^{*}$. Then

$c B^{*}=P\left(\mathbb{R}^{N}\right) \cap S^{*}$, and $S^{*}$ is countably iterable in $N$, and $S^{*}$ is a union of mice projecting to $\mathbb{R}^{N}$. It follows that

$$
S^{*}=Q \mid \xi
$$

for some $\xi$.

Claim 2. $L\left[S^{*}\right] \cap P\left(\mathbb{R}^{N}\right)=\mathcal{B}^{*}$.

Proof. (Let $\Omega$ be a nonselfdual pointclass with the prewellordering property, closed under $\forall^{\mathbb{R}}$, properly included in the projective-in- $\langle A, U\rangle$ sets, with $A, U$, and $S_{0}$ in $\Omega$. Let $\kappa$ be the prewellordering ordinal of $\Omega$, and let $\mu$ be the $\omega$-club ultrafilter on $\kappa$. We have that $L[\mu, S] \cap P(\mathbb{R})=\mathcal{B}$, because otherwise the desired $M$ witnessing the theorem would just be the first level of $L[\mu, S]$ over which a set of reals not in $\mathcal{B}$ is definable, as in the proof of claim 1. It follows that $L[S]$ has a club-in- $\kappa$ class of indiscernibles, in the language with names for each element of $S \cup\{S\}$. The type of this set of indiscernibles in projective-in- $\langle A, U\rangle$, by 
the Coding Lemma. This enables to express the fact that $L[S] \cap P(\mathbb{R})=\mathcal{B}$ as a first order statement about $(\mathrm{HC}, \in, A, U)$. Since $\left(\mathrm{HC}^{N}, \in, A^{*}, U^{*}\right) \prec(\mathrm{HC}, \in, A, U)$, we have proved Claim 2.

Claim 3. Let $\gamma$ be least such that $P\left(\mathbb{R}^{N}\right) \cap Q \mid(\gamma+1) \nsubseteq \mathcal{B}^{*}$; then $\mathcal{Q} \mid(\gamma+1) \models \mathrm{AD}$.

Proof.Work in $N$. Let $\xi$ be such that $S^{*}=Q \mid \xi$; then $\xi$ is the $\theta$ of $Q \mid \gamma$. We have that $Q \mid \gamma \models$ AD. Assume $Q \mid(\gamma+1) \not \models$ AD; then $\gamma$ ends a proper $\Sigma_{1}$ gap in $Q$, and moreover this gap is weak. Let $n$ be least such that $\rho_{n}(Q \mid \gamma)=\mathbb{R}^{N}$. By the analysis of scales in $K(\mathbb{R})$ at the end of a weak gap (see [26]), every boldface $\boldsymbol{\Sigma}_{\mathbf{n}}^{\mathbf{Q} \mid \gamma}$ set of reals $D$ can be written

$$
D=\bigcup_{n<\omega} D_{n}
$$

where each $D_{n} \in \mathcal{B}^{*}$. But then each such $D$ is projective in $A^{*}$. Hence every set of reals in $Q \mid(\gamma+1)$ is projective in $A^{*}$. As $\left(\mathrm{HC}^{N}, \in, A^{*}, U^{*}\right) \prec(\mathrm{HC}, \in, A, U)$, each such set is determined, contradiction.

We need now a slight refinement of Woodin's result that if $G$ and $H$ are models of AD $^{+}$ containing all the reals, and they diverge, in that each has a set of reals not in the other, then every game on $\mathbb{R}$ with payoff in $G \cap H$ has a winning strategy in $G \cap H$. We need this for $G$ and $H$ being merely projectively closed:

Theorem 17.2 (Woodin, unpublished) Let $B, C \subseteq \mathbb{R}$, and let $G$ and $H$ be transitive, rudimentarily closed sets such that $B \in G \backslash H$ and $C \in H \backslash G$. Suppose $G \models \mathrm{AD}^{+}$and $H \models \mathrm{AD}^{+}$. Let $D \subseteq \mathbb{R}^{\omega}$ be in $G \cap H$; then the real game with payoff $D$ has a winning strategy in $G \cap H$.

We apply Woodin's theorem in $N$, with $G=Q \mid(\gamma+1)$, where $\gamma$ is as in claim 3, and $H$ the rudimentary closure of $\mathbb{R}^{N} \cup\left\{A^{*}\right\}$. Note here

Claim 4. $S^{*} \forall \mathrm{AD}_{\mathbb{R}}$.

Proof. Otherwise, every set of reals in $S^{*}$ has a scale in $S^{*}$. By the analysis of scales in iterable $\mathbb{R}$-mice $([24])$, new $\Sigma_{1}$ statements about reals are verified cofinally in $S^{*}$. (These statements can refer to the extender sequence of $S^{*}$.) It follows that $\rho_{1}\left(S^{*}\right)=\mathbb{R}^{N}$. That contradicts claim 2 .

By Woodin's theorem, claim 4 , and the minimality of $A^{*}$ and $\gamma$ respectively, we get that the subsets of $\mathbb{R}^{N}$ in $Q \mid(\gamma+1)$ are precisely those which (in $N$ ) are projective in $A^{*}$. Thus if $M=Q \mid(\gamma+1)$, then

$$
\begin{aligned}
N \models & M \text { is sound, projects to } \mathbb{R} \text {, and } \\
& A^{*} \text { is definable over } M \text {, and the theory }
\end{aligned}
$$


of $M$ with real parameters is projective in $\left\langle A^{*}, U^{*}\right\rangle$, and

every countable elementary submodel of $M$ has an

$\omega_{1}$ iteration strategy coded by a set of reals $\left.<_{w} U^{*}\right)$.

This contradiction completes the proof of 17.1 .

Corollary 17.3 Assume $\mathrm{AD}^{+}+\mathrm{MSC}$. Let $U$ be the universal $\Sigma_{1}^{2}$ set of reals, and $A$ any $O D(\mathbb{R})$ set of reals; then there is a countably iterable $\mathbb{R}$-mouse $M$ such that $A$ is definable over $M$, and every set of reals definable over $M$ is projective in $A$ and $U$.

Corollary 17.4 Assume $\mathrm{AD}^{+}+\mathrm{MSC}$. Let $A$ be set of reals; then there is an $\mathbb{R}$-premouse $M$ such that $A$ is definable over $M$, and every set of reals definable over $M$ is projective in $A$ and $U$.

There are extensions of theorem 17.1 and its corollaries to hybrid strategy-mice: capturing over countable sets of reals implies capturing over $\mathbb{R}$. The key to formulating these is to notice that if $\Sigma$ is an $\omega_{1}$-iteration strategy for some countable $M$, and $\Sigma$ condenses well, then there is at most one extension of $\Sigma$ to uncountable iteration trees. So one can define a $\Sigma$-mouse over $\mathbb{R}$ to be a hybrid mouse all of whose countable elementary submodels containing $M$ are hybrid $\Sigma$-mice over their own (countable) set of reals. One can use this to make sense of $K^{\Sigma}(\mathbb{R})$, and to generalize 17.1 .

The results of this section should be compared to those at the beginning of section 4 .

\section{References}

[1] Q. Feng, M. Magidor, and W.H. Woodin, Universally Baire sets of reals, in Set theory of the Continuum, H. Judah, W. Just, and W.H. Woodin eds., MSRI publications vol 26 (1992), Springer-Verlag.

[2] L.A. Harrington and A.S. Kechris, On the determinacy of games on ordinals, Annals of Math. Logic, vol. 20 (1981), 109-154.

[3] S. Jackson, Structural consequences of AD, Handbook of set theory, M. Foreman, A. Kanamori, and M. Magidor eds., to appear.

[4] R. Ketchersid, Toward $A D_{\mathbb{R}}$ from the Continuum Hypothesis and an $\omega_{1^{-}}$dense ideal, Ph.D. thesis, Berkeley, 2000.

[5] P. Koellner and W.H. Woodin, Large cardinals from determinacy, Handbook of Set Theory, M. Foreman, A. Kanamori, and M. Magidor eds., to appear. 
[6] P. Larson, The stationary tower, Memoirs of the AMS.

[7] D.A. Martin, Measurable cardinals and analytic games, Bulletin of the AMS, 1968.

[8] D.A. Martin, The largest countable this, that, and the other, in Cabal Seminar 79-81, A.S. Kechris, D.A. Martin, and Y.N. Moschovakis eds., Lecture Notes in Math, vol. 1019 (1983), Springer-Verlag, Berlin, 97-106.

[9] D.A. Martin and R.M. Solovay, A basis theorem for $\Sigma_{3}^{1}$ sets of reals, Annals of Mathematics 89 (1969), 138-159.

[10] D.A. Martin and J.R. Steel, A Proof of Projective Determinacy, Journal of the American Mathematical Society bf 2 (1989), 71-125.

[11] W.J. Mitchell and R.D. Schindler, A universal extender model without large cardinals in $V$, J. Symb. Logic, vol. 69 (2004), pp. 371-386.

[12] W.J. Mitchell and J.R. Steel, Fine structure and iteration trees, Lecture Notes in Logic 3, Springer-Verlag, Berlin 1994.

[13] I. Neeman, Inner models in the region of a Woodin limit of Woodin cardinals, Ann. of Pure and Applied Logic, vol. 116 (2002) pp. 67-155.

[14] R.D. Schindler and J.R. Steel, The strength of AD, unpublished but available at http://www.math.uni-muenster.de/math/inst/logik/org/staff/rds.

[15] R.D. Schindler and J.R. Steel, The self-iterability of $L[\vec{E}]$, Journal of Symb. Logic, to apppear.

[16] R.D. Schindler and J.R.Steel, Problems in inner model theory, available at http://www.math.uni-muenster.de/math/inst/logik/org/staff/rds.

[17] F. Schlutzenberg and J.R. Steel, Comparing fine mice via coarse iteration, in preparation.

[18] T.A. Slaman and J.R. Steel, Definable functions on degrees, Cabal Seminar 81-85, A. Kechris, D. Martin, J. Steel eds., Springer Lecture Notes in Mathematics vol. 1333, pp. $37-55$.

[19] J.R. Steel, An outline of inner model theory, Handbook of Set Theory, M. Foreman, A. Kanamori, and M. Magidor eds., to appear.

[20] J.R. Steel, The core model iterability problem , Lecture Notes in Logic 8, SpringerVerlag, Berlin 1996. 
[21] J.R. Steel, Local $K^{c}$ constructions, Journal of Symb. Logic, to appear.

[22] J.R. Steel, Woodin's analysis of $\mathrm{HOD}^{L(\mathbb{R})}$, unpublished, available at http://www.math.berkeley.edu/ steel

[23] J. R. Steel, A theorem of Woodin on mouse sets, unpublished, available at http://www.math.berkeley.edu/ steel

[24] J.R. Steel, Scales in $K(\mathbb{R})$, to appear in The New Cabal, available at http://www. math.berkeley.edu/ steel

[25] J.R. Steel, The derived model theorem, unpublished, available at http://www.math.berkeley.edu/ steel.

[26] J.R. Steel, Scales at the end of a weak gap, Journal of Symb. Logic, to appear.

[27] J.R. Steel, PFA implies AD ${ }^{L(\mathbb{R})}$, Journal of Symb. Logic vol. 70 (2005) 1255-1296.

[28] J.R. Steel, A classification of jump operators, Journal of Symb. Logic vol. 47 (1982) $347-358$.

[29] W.H. Woodin, unpublished lecture notes, Berkeley 1993-94.

[30] W.H. Woodin, Supercompact cardinals, sets of reals, and weakly homogeneous trees,Proc. Nat. Acad. Sci. USA 85, 6587-6591.

[31] M. Zeman, Inner models and large cardinals, de Gruyter Series in Logic and Applications, vol. 5, Berlin 2002.

[32] A.S. Zoble, Stationary reflection and the determinacy of inductive games, Ph.D. thesis, U.C. Berkeley (2000). 